quatrième série-tome 41 fascicule 6 novembre-décembre 2008

$$
\begin{aligned}
& \text { ANNALES } \\
& \text { SCIENTIFIQUES } \\
& \text { de } \\
& \text { L'ÉCOLE } \\
& \text { NORMALE } \\
& \text { SUPERIEURE }
\end{aligned}
$$

Nader MASMOUDI \& Frédéric ROUSSET

Stability of oscillating boundary layers in rotating fluids 
Ann. Scient. Éc. Norm. Sup.

$4^{\mathrm{e}}$ série, t. 41, 2008, p. 955 à 1002

\title{
STABILITY OF OSCILLATING BOUNDARY LAYERS IN ROTATING FLUIDS
}

\author{
BY NADER MASMOUdi ANd Frédéric ROUSSET
}

Abstract. - We prove the linear and non-linear stability of oscillating Ekman boundary layers for rotating fluids in the so-called ill-prepared case under a spectral hypothesis. Here, we deal with the case where the viscosity and the Rossby number are both equal to $\varepsilon$. This study generalizes the study of [23] where a smallness condition was imposed and the study of [26] where the well-prepared case was treated.

RÉSUMÉ. - On prouve la stabilité linéaire et non-linéaire de couches limites oscillantes de type Ekman pour les fluides tournant dans le cas de données mal préparées sous une hypothèse spectrale. On s'intéresse au cas où la viscosité et le nombre de Rossby sont du même ordre $\varepsilon$. Cette étude généralise celle de [23] où une condition de petitesse était imposée et celle de [26] où les données bien préparées étaient traitées.

\section{Introduction}

We consider the following system describing the evolution of a rotating fluid in a rectangular domain

$$
\left\{\begin{array}{l}
\partial_{t} u^{\varepsilon}+u^{\varepsilon} \cdot \nabla u^{\varepsilon}+\frac{e \times u^{\varepsilon}}{\varepsilon}+\frac{\nabla p}{\varepsilon}-\varepsilon \Delta u^{\varepsilon}=0, \\
\nabla \cdot u^{\varepsilon}=0
\end{array}\right.
$$

for $x=(y, z) \in \Omega=\mathbb{T}_{a}^{2} \times(0,1)$ with the Dirichlet boundary condition

$$
u_{/ \partial \Omega}^{\varepsilon}=0
$$

and the initial condition

$$
u_{/ t=0}^{\varepsilon}=u^{\varepsilon, 0} \text {. }
$$

Here $\mathbb{T}_{a}^{2}$ is the periodic torus with periods $a_{1}$ and $a_{2}$, namely, $\mathbb{T}_{a}^{2}=\mathbb{R}^{2} /\left(a_{1} \mathbb{Z} \times a_{2} \mathbb{Z}\right)$ and $a_{1}, a_{2}>0$. Moreover, $e=e_{3}$ is the vertical unit vector and $\frac{e \times u^{\varepsilon}}{\varepsilon}$ is the Coriolis force. 
This system describes the motion of a rotating fluid as the Ekman and Rossby numbers go to zero (see Pedlovsky [25], and Greenspan [14]). It can model the dynamics of the ocean or the atmosphere far from the equator or a rotating fluid in a container. Note that, here, we take the horizontal viscosity and the vertical viscosity to be equal. We point out that in many previous works the horizontal viscosity was supposed constant whereas the vertical viscosity $\nu$ goes to 0 (see for instance [17]) or in some other cases, the vertical viscosity was supposed much smaller than the horizontal viscosity. This anisotropy has the advantage of making the boundary layers more stable.

In this paper, we look at the case where the vertical and the horizontal viscosities are equal. We study the convergence of solutions to (1) towards a solution of the limit system ( 9 ) defined below once the time oscillations are filtered out.

We recall that this system and related ones were studied by several authors. In the "wellprepared" case in domains with boundary, like $\Omega$, we refer to Colin, Fabrie [5], Grenier, Masmoudi [17], Masmoudi [22]. For general initial data, and for the periodic case, we refer to Grenier [15], Embid and Majda [9], Babin, Mahalov and Nicolaenko [2, 1], Gallagher [12] or in particular cases where there is no boundary layer, or where the boundary layer can be eliminated by symmetry (Beale and Bourgeois [3]). These results rely on the introduction of a group to filter the oscillations in time, a method which was previously used by Schochet [30] to investigate related problems in the torus concerning the compressible-incompressible limit.

In [23], the "group method" was extended to the case of domains with boundary, by solving a superposition of an infinite number of boundary layers. These layers create an extra term in the limit equation. In [23], the stability of these boundary layers was proved in the case where the horizontal viscosity goes to zero slower than the Rossby number (or in the small data case). In this paper, we would like to give a spectral assumption (which we think is optimal) and which yields the stability of such boundary layers.

In the well prepared case, a similar spectral assumption was used to prove the stability of the boundary layer [26]. This spectral assumption is optimal since the instability of the boundary layer was proved in [7] if the spectral assumption does not hold.

In the following sections, we recall the main properties of the approximate solution of (1) constructed in [23] (see also [4]), in particular, we recall the properties of the limit system, of the boundary layers and the assumptions on the torus which are needed. Next, we shall give our main assumption on the spectral stability of the boundary layers and state our main result.

\section{Acknowledgement}

N. Masmoudi was partially supported by an NSF grant DMS-0703145. This work was carried out during visits of $\mathrm{N}$. Masmoudi to the university of Nice and visits of F. Rousset to the Courant Institute. The hospitality of both Institutes is highly acknowledged. 


\subsection{Properties of the approximate solution}

To state our main result, we first recall the main properties of the approximate solution $u^{\text {app }}$ of (1) constructed in [23]. In particular, $u^{\text {app }}$ describes the formal limit of (1) and the boundary layers. The details of the construction will be recalled later. The approximate solution is under the form

$$
u^{\mathrm{app}}=u^{\mathrm{int}}\left(\frac{t}{\varepsilon}, t, x\right)+u^{b}\left(\frac{t}{\varepsilon}, \frac{z}{\varepsilon}, \frac{1-z}{\varepsilon}, t, y\right)+u^{r}, \quad x=(y, z) \in \mathbb{T}_{a}^{2} \times(0,1)
$$

where the remainder term $u^{r}$ satisfies $u^{r}=\mathcal{O}(\varepsilon)$ (a precise statement will be given later). The interior term $u^{\text {int }}$ can be expressed as

$$
u^{\text {int }}(\tau, t, x)=\mathcal{L}(\tau) w^{\text {int }}(t, x)
$$

where $\mathcal{L}(\tau)=e^{\tau L}, L u=-\mathbb{P}(e \times u)$ and $\mathbb{P}$ is the Leray projector on divergence-free vector fields with zero normal component in $\Omega$. We denote $\mathbb{Z}_{a}^{3}=\frac{2 \pi}{a_{1}} \mathbb{Z} \times \frac{2 \pi}{a_{2}} \mathbb{Z} \times \frac{2 \pi}{2} \mathbb{Z}, \mathbb{Z}_{a}^{2}=\frac{2 \pi}{a_{1}} \mathbb{Z} \times \frac{2 \pi}{a_{2}} \mathbb{Z}$ and we denote elements of $\mathbb{Z}_{a}^{3}$ by $\bar{k}=\left(k, k_{3}\right) \in \mathbb{Z}_{a}^{3}$ with $k \in \mathbb{Z}_{a}^{2}=\frac{2 \pi}{a_{1}} \mathbb{Z} \times \frac{2 \pi}{a_{2}} \mathbb{Z}$. We have an expansion

$$
w^{\mathrm{int}}(t, x)=\sum_{\bar{k} \in \mathbb{Z}_{a}^{3}} b(t, \bar{k}) e^{i k \cdot y} M^{\bar{k}}(z)
$$

so that

$$
u^{\mathrm{int}}(\tau, t, x)=\mathcal{L}(\tau)\left(w^{\mathrm{int}}(t, x)\right)=\sum_{\bar{k} \in \mathbb{Z}_{a}^{3}} b(t, \bar{k}) e^{i k \cdot y} M^{\bar{k}}(z) e^{i \lambda(\bar{k}) \tau}
$$

and $w^{\text {int }}$ solves the limit system (9). Note that $N^{\bar{k}}=e^{i k \cdot y} M^{\bar{k}}$ is an eigenvector of $L$. We assume that the initial data is chosen such that $b\left(0,\left(0, k_{3}\right)\right)=0$ for every $k_{3}$ i.e. we exclude initial values with modes which depend only on $z$. We shall also assume that the torus is non resonant in the sense of [2] to insure that the condition $b\left(t,\left(0, k_{3}\right)\right)=0$ for every $k_{3}$ remains true for positive times (see below for a precise definition).

We can express the dominant boundary layer term $u^{b}$ as

$$
u^{b}\left(\tau, Z, Z^{\prime}, t, y\right)=u^{b, 0}(\tau, Z, t, y)+u^{b, 1}\left(\tau, Z^{\prime}, t, y\right)
$$

where

$$
u^{b, \sigma}(\tau, Z, t, y)=-\frac{1}{2} \sum_{\bar{k}} b(t, \bar{k}) e^{i k \cdot y+i \lambda(\bar{k}) \tau}(-1)^{\sigma k_{3}}\left(h^{\bar{k},+} e^{-\frac{1+i}{\sqrt{2}} \eta^{\bar{k},+} Z}+h^{\bar{k},-} e^{-\frac{1-i}{\sqrt{2}} \eta^{\bar{k},-} Z}\right)
$$

for $\sigma=0,1$, with

$$
\eta^{\bar{k}, \pm}=\sqrt{1 \pm \lambda(\bar{k})}, \quad h^{\bar{k}, \pm}=M^{\bar{k}}(0) \mp i e \times M^{\bar{k}}(0) .
$$

Note that since terms under the form $\left(0, k_{3}\right)$ are excluded in the above sum, we have $\eta^{\bar{k}, \pm}>0$ and hence, we have a superposition of terms which are small far from the boundary. Nevertheless, the rate of decay, $\eta^{\bar{k}, \pm}$, goes to zero when $\frac{k_{3}}{|k|}$ tends to \pm 1 .

In view of the above definition of the boundary layers, we introduce the operators

$$
\mathcal{B}^{\sigma}(\tau, Z) q=-\frac{1}{2} \sum_{\bar{k}} q_{\bar{k}} e^{i \lambda(\bar{k}) \tau}(-1)^{\sigma k_{3}}\left(h^{\bar{k},+} e^{-\frac{1+i}{\sqrt{2}} \eta^{\bar{k},+} Z}+h^{\bar{k},-} e^{-\frac{1-i}{\sqrt{2}} \eta^{\bar{k},-} Z}\right)
$$

for any sequence $q=\left(q_{\bar{k}}\right)_{\bar{k} \in \mathbb{Z}_{a}^{3}}$ so that if $q$ is taken under the form $q_{\bar{k}}=b(t, \bar{k}) e^{i k \cdot y}$, we have $\mathcal{B}^{\sigma}(\tau, y, Z) q=u^{b, \sigma}(\tau, Z, t, y)$. 
In a similar way, we also define

$$
\mathcal{L}^{\sigma}(\tau) q=\sum_{\bar{k}} q_{\bar{k}} M^{\bar{k}}(\sigma) e^{i \lambda(\bar{k}) \tau}, \quad \sigma=0,1 .
$$

Again, note that if $q$ is taken such that $q_{\bar{k}}=b(t, \bar{k}) e^{i k \cdot y}$, then we have $\mathcal{L}^{\sigma}(\tau) q=w(t, y, \sigma)$.

We shall allways assume that the initial data is sufficiently smooth and vanishes at a sufficient order at $z=0, z=1$ in order that $b(t, \bar{k})$ decay to zero sufficiently fast. In particular, we assume that

$$
\left\|w^{0}\right\|_{V_{\text {sym }}^{s}}^{2}=\sum_{\bar{k} \in \mathbb{Z}_{a}^{3}}|b(0, \bar{k})|^{2}|\bar{k}|^{2 s}<\infty \quad \text { for some } s \text { big enough. }
$$

This yields that $w(t) \in V_{\text {sym }}^{s}$ for $0<t<T^{*}$ where $T^{*}$ is the life span of a smooth solution of the limit system (9). Hence, by using that $s>\frac{3}{2}+2$, we have since

$$
\left(\frac{1}{\eta^{\bar{k}, \pm}}\right)^{2} \leq \frac{|\bar{k}|}{|\bar{k}|-\left|k_{3}\right|}=\frac{2|\bar{k}|^{2}}{k_{1}^{2}+k_{2}^{2}} \leq 2|\bar{k}|^{2}
$$

that

$$
\sum_{\bar{k}}|b(t, \bar{k})|\left(1+\left(\frac{1}{\eta^{\bar{k},+}}\right)^{2}+\left(\frac{1}{\eta^{\bar{k},-}}\right)^{2}\right)<\infty
$$

to finally obtain the important property

$$
\sup _{y} \int_{0}^{+\infty}\left|\partial_{Z} \mathcal{B}^{\sigma}(\tau, Z)(w(t, y, \sigma))\right|\left(1+|Z|+\left|Z^{2}\right|\right) d Z<+\infty, \quad \sigma=0,1
$$

which insures that the boundary layers are sufficiently localized in the vicinity of the boundary.

\subsection{The limit system}

We denote by $w^{\text {int }}=\sum_{\bar{k} \in \mathbb{Z}_{a}^{3}} b(t, \bar{k}) N^{\bar{k}}$ the solution in $L^{\infty}\left(0, T^{*} ; V_{\mathrm{sym}}^{s}\right)$ of the following system

$$
\left\{\begin{array}{l}
\partial_{t} w^{\mathrm{int}}+\bar{Q}\left(w^{\mathrm{int}}, w^{\mathrm{int}}\right)+\bar{S}\left(w^{\mathrm{int}}\right)=-\nabla p \quad \text { in } \Omega \\
\nabla \cdot w^{\mathrm{int}}=0 \quad \text { in } \Omega \\
w^{\mathrm{int}} \cdot n= \pm w_{3}=0 \quad \text { on } \partial \Omega \\
w^{\mathrm{int}}(t=0)=w^{0}
\end{array}\right.
$$

where $T^{*}$ is the time of existence of the smooth solution $w^{\text {int }}$ of (9) and $\bar{Q}\left(w^{\text {int }}, w^{\text {int }}\right), \bar{S}\left(w^{\text {int }}\right)$ are respectively a bilinear and a linear operator of $w^{\text {int }}$.

The bilinear operator is given by

$$
\bar{Q}\left(w^{\text {int }}, w^{\text {int }}\right)=\sum_{\substack{\bar{l}, \bar{m}, \bar{k} \\ \bar{k} \in \mathcal{A}(\bar{l}, \bar{m}) \\ \lambda(\bar{l})+\lambda(\bar{m})=\lambda(\bar{k})}} b(t, \bar{l}) b(t, \bar{m}) \alpha_{\bar{l} \bar{m} \bar{k}} N^{\bar{k}}(X) .
$$

The numbers $\alpha_{\bar{l} \bar{m} \bar{k}}$ are constants and the set $\mathcal{A}(\bar{l}, \bar{m})=\{\bar{l}+\bar{m}, S \bar{l}+\bar{m}, \bar{l}+S \bar{m}, S \bar{l}+S \bar{m}\}$ with the notation

$$
S\left(\bar{l}_{1}, \bar{l}_{2}, \bar{l}_{3}\right)=\left(\bar{l}_{1}, \bar{l}_{2},-l_{3}\right)
$$

is the set of possible resonances.

$4^{\mathrm{e}}$ SÉRIE - TOME $41-2008-\mathrm{N}^{\circ} 6$ 
The linear operator is defined by

$$
\bar{S}\left(w^{\text {int }}\right)=\sum_{\bar{k}}(D(\bar{k})+i I(\bar{k})) b(t, \bar{k}) N^{\bar{k}}(X)
$$

where

$$
D(\bar{k})=\sqrt{2}\left\{\left(1-\lambda(\bar{k})^{2}\right)^{\frac{1}{2}}\right\}, \quad I(\bar{k})=\sqrt{2}\left\{\lambda(\bar{k})\left(1-\lambda(\bar{k})^{2}\right)^{\frac{1}{2}}\right\} .
$$

Note that $\bar{S}\left(w^{\text {int }}\right)$ is a damping term, since $D(\bar{k}) \geq 0$, that depends on the frequencies $\lambda(\bar{k})$.

\subsection{Non resonance assumption on the torus}

In the case of a non resonant torus (see [2] for the definition), the quadratic term $\bar{Q}(w, w)$ only includes trivial resonances, namely the resonances only take place with the $2 d$ non oscillating geostrophic part:

$$
\{(\bar{k}, \bar{l}, \bar{m}) \mid \bar{k} \in \mathcal{A}(\bar{l}, \bar{m})\} \subset\left\{(\bar{k}, \bar{l}, \bar{m}) \mid k_{3} m_{3} l_{3}=0\right\}
$$

which yields in particular the global existence of strong solutions to the limit system. We also know in that case that for $\bar{k} \in \mathcal{A}(\bar{l}, \bar{m})$, and $\lambda(\bar{l})+\lambda(\bar{m})-\lambda(\bar{k}) \neq 0$

$$
\frac{1}{\lambda(\bar{l})+\lambda(\bar{m})-\lambda(\bar{k})} \leq C\left(|\bar{l}|^{d}+|\bar{m}|^{d}\right)
$$

for some $d>4$. We recall that for almost all choices of $a_{1}$ and $a_{2}$, the torus $\mathbb{T}_{a}^{3}$ is non resonant (see [2]).

Besides, if at $t=0$, we have

$$
\int_{x, y} w^{0} d x d y=0
$$

we see that this holds for any $t$. Indeed, in the non resonant case there are only trivial resonances, namely with the slow modes (the geostrophic modes) $\left(k_{1}, k_{2}, 0\right)$. Notice then that the modes $\left(k_{1}, k_{2}, 0\right)$, and $\left(-k_{1},-k_{2}, k_{3}\right)$ do not create a resonance with $\left(0,0, k_{3}\right)$, since $\left(k_{1}, k_{2}\right) \neq(0,0)$, and then $\left|\lambda\left(-k_{1},-k_{2}, k_{3}\right)\right|<1$. Hence we get that for all $t$ the modes such that $\lambda(\bar{k})= \pm 1$ are absent. This is a crucial fact in our analysis since the boundary layers for the modes $\lambda(\bar{k})= \pm 1$ behave like the boundary layers in the vanishing viscosity limit of the Navier-Stokes equation without the fast rotation (these layers are of Prandtl-type). For such a case, the stability of the boundary layer is known if the horizontal viscosity is much bigger than the vertical one (see [22]), in other cases instability is more expected [16] except in dimension 1 [27] or for analytic data [29].

\subsection{Stability assumption on the boundary layer profiles}

The main difficulty in the convergence proof is to get an estimate for (1) linearized about the approximate solution $u^{\text {app }}$ : we study

$$
\partial_{t} v+u^{\text {app }} \cdot \nabla v+v \cdot \nabla u^{\text {app }}-\varepsilon \Delta v+\nabla p+\frac{e \times v}{\varepsilon}=0, \quad x \in \mathbb{T}_{a}^{2} \times(0,1)
$$

with the boundary condition (2) and the initial condition $v_{/ t=0}=v_{0}(x)$. We would like to prove an estimate like

$$
\|v(T)\|^{2} \leq e^{\gamma T}\left\|v_{0}\right\|^{2}
$$

for some norm $\|\cdot\|$ with $\gamma>0$ independent of $\varepsilon$. Even in the well-prepared case such an estimate is not always true, it depends on a spectral stability property of the boundary layer 
profiles. If the boundary layer profiles are spectrally stable, this estimate can be proven as well as nonlinear stability, [26]. Whereas if they are unstable, we can only get an estimate with $\gamma$ of the order of $\varepsilon^{-1}$ and in this case nonlinear instability can be proven [7]. The spectral stability depends on the amplitude of the boundary layer, numerically, one can prove that boundary layers with too large amplitude are unstable [21]. The aim of the next subsection is to formulate a stability assumption on the boundary layer profiles which generalize the spectral stability assumption of the well-prepared case formulated in [26].

We start by freezing the slow variables $t=t^{0}$ and $y=y^{0}$ in the coefficients of the approximate solution. Let us set $q=q\left(t^{0}, y^{0}\right)=\left(b\left(t^{0}, \bar{k}\right) e^{i k \cdot y^{0}}\right)_{\bar{k} \in \mathbb{Z}_{a}^{3}}$. We want to study the stability property of the equations

$\partial_{t} v+\left(\mathcal{L}^{\sigma}\left(\frac{t}{\varepsilon}\right) q+\mathcal{B}^{\sigma}\left(\frac{t}{\varepsilon}, \frac{z}{\varepsilon}\right) q\right) \cdot \nabla v+v \cdot \nabla\left(\mathcal{L}^{\sigma}\left(\frac{t}{\varepsilon}\right) q+\mathcal{B}^{\sigma}\left(\frac{t}{\varepsilon}, \frac{z}{\varepsilon}\right) q\right)+\frac{\nabla p}{\varepsilon}+\frac{e \times v}{\varepsilon}-\varepsilon \Delta v=0$.

Let us define for each sequence $q=\left(q_{\bar{k}}\right)_{\bar{k} \in \mathbb{Z}_{a}^{3}}$ the oscillating boundary layer profile $V(\tau, Z, q)$ as

$$
V^{\sigma}(\tau, Z, q)=\mathcal{L}^{\sigma}(\tau) q+\mathcal{B}^{\sigma}(\tau, Z) q
$$

we can take the Fourier transform in $y$ and set $Z=z / \varepsilon$ and $\tau=t / \varepsilon$ to get the family of one-dimensional problems

$$
\begin{gathered}
\partial_{\tau} w+V^{\sigma}(\tau, Z, q) \cdot\left(\begin{array}{c}
i \varepsilon k \\
\partial_{Z}
\end{array}\right) w+w \cdot\left(\begin{array}{c}
i \varepsilon k \\
\partial_{Z}
\end{array}\right) V^{\sigma}(\tau, Z, q) \\
+\left(\begin{array}{c}
i \varepsilon k \\
\partial_{Z}
\end{array}\right) p+e \times w+\varepsilon^{2}|k|^{2} w-\partial_{Z Z} w=0, \\
i \varepsilon k \cdot w_{h}+\partial_{Z} w_{3}=0
\end{gathered}
$$

which is now set for $Z \in(0,+\infty)$ with the boundary condition

$$
w(\tau, \varepsilon k, 0)=0 .
$$

We recall here that we use the notation $\bar{k}=\left(k, k_{3}\right)$ where $k \in \mathbb{Z}_{a}^{2}$. Finally, we can set $\xi=\varepsilon k$ and use for $\xi \neq 0$ the Leray projection $\mathbb{P}_{+}(\xi)$ in the half-space which is recalled in section 19 to rewrite the equation as

$$
\partial_{\tau} w=\mathbb{P}_{+}(\xi) \mathbb{L}_{+}^{\sigma}(\xi, q) w, \quad i \xi \cdot w_{h}+\partial_{Z} w_{3}=0
$$

where $\mathbb{L}_{+}^{\sigma}(\tau, \xi, q)$ is defined as

$$
\mathbb{L}_{+}^{\sigma}(\tau, \xi, q) w=-V^{\sigma}(\tau, Z, q) \cdot\left(\begin{array}{c}
i \xi \\
\partial_{Z}
\end{array}\right) w-w \cdot\left(\begin{array}{c}
i \xi \\
\partial_{Z}
\end{array}\right) V^{\sigma}(\tau, Z, q)-e \times w-|\xi|^{2} w+\partial_{Z Z} w
$$

The non-autonomous operator $\mathbb{P}_{+} \mathbb{L}_{+}^{\sigma}(\tau, \xi, q)$ generates a strongly continuous family of evolution operators in the sense of [20], Chapter 7, $\mathbb{S}_{+}^{\sigma}\left(\tau, \tau^{\prime}, \xi, q\right)$ on $H_{\xi}=\left\{w \in L^{2}(0,+\infty)\right.$, $\left.i \xi \cdot w_{h}+\partial_{Z} w_{3}=0\right\}$. As usual, the main property of $\mathbb{S}_{+}^{\sigma}\left(\tau, \tau^{\prime}, \xi, q\right)$ is that $\tau \mapsto$ $\mathbb{S}_{+}^{\sigma}\left(\tau, \tau^{\prime}, \xi, q\right) w_{0}$ is the unique solution of (15) for $\tau>\tau^{\prime}$ with value $w_{0}$ for $\tau=\tau^{\prime}$.

Let us fix $s_{0}>1$ such that (8) holds when we replace $w(t, y, \sigma)$ by $\mathcal{L}^{\sigma}(\tau) q$. A set $\mathcal{K} \subset$ $V_{\mathrm{sym}}^{s_{0}}(\Omega)$ or more precisely in $\left\{\left.q\left|\sum_{\bar{k} \in \mathbb{Z}_{a}^{3}}\right| \bar{k}\right|^{2 s_{0}}\left|q_{\bar{k}}\right|^{2}<\infty\right\}$ (using the identification between 
the function and its Fourier coefficients) will be called a uniform stability set if for every $r, R$, $0<r<R$, there exist $C(r, R)$ and $\alpha(r, R)>0$ such that

$$
\forall v \in H_{\xi}, \quad\left|\mathbb{S}_{+}^{\sigma}\left(\tau, \tau^{\prime}, \xi, q\right) v\right|_{L^{2}\left(\mathbb{R}_{+}\right)} \leq C e^{-\alpha\left(\tau-\tau^{\prime}\right)}|v|_{L^{2}\left(\mathbb{R}_{+}\right)}, \quad \forall \tau \geq \tau^{\prime} \geq 0, \sigma=0,1
$$

for every $q \in \mathcal{K}$ and $\xi$ such that $r \leq|\xi| \leq R$.

We point out that there are uniform stability sets. Indeed, a vicinity of zero is a uniform stability set since we can prove as in $[23,6]$ that all weak amplitude boundary layers are stable when (8) is matched.

Let $w^{\text {int }}(t)=\sum_{\bar{k} \in \mathbb{Z}_{a}^{3}} b(t, \bar{k}) N^{\bar{k}}$ be the solution of (9). Our main stability assumption is:

(H) We assume that the set $\mathcal{K}=\left\{\left(q_{k}(t, y):=b(t, \bar{k}) e^{i k \cdot y}\right)_{\bar{k} \in \mathbb{Z}_{a}^{3}}, t \in[0, T], y \in \mathbb{T}_{a}^{2}\right\}$, is a uniform stability set.

REMARK 1. - Note that we can define more generally the stability set $\mathcal{K}_{0} \subset V_{\text {sym }}^{s_{0}}(\Omega)$ as the set of $q$ having the property that for every $0<r<R$, there exist $C(r, R, q)>0$ and $\alpha(r, R, q)>0$ such that (17) holds. By continuity of $\mathbb{S}_{+}$with respect to $q$ and compactness, we easily get that every compact set $\mathcal{K} \subset \mathcal{K}_{0}$ is a uniform stability set. In particular, this yields that for every bounded set $B \subset V_{\text {sym }}^{s}(\Omega)$ with $s>s_{0}$, we have that if $B$ is included in $\mathcal{K}_{0}$, then $B$ is a uniform stability set. Consequently, the assumption $(\mathrm{H})$ is matched if $\mathcal{K} \subset \mathcal{K}_{0}$ (where $\mathcal{K}$ is defined in the statement of $(\mathrm{H})$ and $\mathcal{K}$ is bounded in $V_{\text {sym }}^{s}(\Omega)$ for $s>s_{0}$.

Since the operator $\mathbb{L}_{+}^{\sigma}$ has only quasi-periodic coefficients there is no easy characterization of the assumption $(\mathrm{H})$, for example in term of the spectrum of $\mathbb{L}_{+}$. When the initial data is prepared so that the coefficients of $\mathbb{L}_{+}^{\sigma}$ are periodic in time, we can use Floquet's theory to replace in the assumption $(\mathrm{H})$ the decay estimate (17) by an assumption on the spectrum of $\mathbb{S}_{+}^{\sigma}(T, 0, \xi, q)$ where $T$ is the period: if the spectrum $\sigma\left(\mathbb{S}_{+}^{\sigma}(T, 0, \xi, q)\right)$ is contained in the open unit disk $\mathbb{D}$, then the estimate (17) is verified. Finally, we note that $(\mathrm{H})$ is the natural generalization of the assumption used in the well-prepared case. Indeed, in the well prepared case, since $\mathbb{L}_{+}^{\sigma}$ does not depend on $\tau$ the stability assumption was formulated in term of the spectrum of $\mathbb{P}_{+}(\xi) \mathbb{L}_{+}^{\sigma}(\xi, q)$ : it was basically assumed in [26] that the spectrum of $\mathbb{P}_{+}(\xi) \mathbb{L}_{+}^{\sigma}(\xi, q)$ is contained in $\{R e \lambda<0\}$. By the standard theory of analytic semi-groups, it is easy to prove that this assumption on the spectrum implies the estimate (17). This is proven in [13] in a close setting.

\subsection{Notations}

We denote by $\|\cdot\|$ the norm of $L^{2}\left(\mathbb{T}_{a}^{2} \times(0,1)\right)$ and by $(\cdot, \cdot)$ the associated scalar product. We also define the weighted higher order norms:

$$
\begin{aligned}
& \|v\|_{1, \varepsilon}^{2}=\|v\|^{2}+\varepsilon^{2}\|\nabla v\|^{2}, \\
& \|v\|_{2, \varepsilon}^{2}=\|v\|^{2}+\varepsilon^{2}\|\nabla v\|^{2}+\varepsilon^{4}\left\|\nabla^{2} v\right\|^{2} .
\end{aligned}
$$

We will also use some anisotropic norms, namely

$$
\|v\|_{m}^{2}=\sum_{\alpha \in \mathbb{Z}^{3},|\alpha| \leq m}\left\|\bar{Z}^{\alpha} v\right\|^{2}
$$

where $\bar{Z}_{1}=\partial_{y_{1}}, \bar{Z}_{2}=\partial_{y_{2}}$, and $\bar{Z}_{3}=\varepsilon z(1-z) \partial_{z}$ and denote by $H_{\text {anis }}^{m}$ the Hilbert space defined by this norm. 


\subsection{Main result}

Our main result is:

Theorem 2. - We consider a torus $\mathbb{T}_{a}^{3}$ non-resonant in the sense of [2] and $w^{0} \in V_{\mathrm{sym}}^{s}$ for $s$ sufficiently large such that in the expansion

$$
w^{0}(x)=\sum_{\bar{k} \in \mathbb{Z}_{a}^{3}} b^{0}(\bar{k}) e^{i k \cdot y} M^{\bar{k}}(z)
$$

we have $b^{0}(\bar{k})=0$ if $\bar{k}=\left(0,0, k_{3}\right)$. Moreover, with the notation $q^{0}(y)=\left(q_{\bar{k}}^{0}(y)\right)_{\bar{k}}=$ $\left(b^{0}(\bar{k}) e^{i k \cdot y}\right)_{\bar{k}}$, we assume that

$$
\left\{\begin{array}{l}
\left\|u^{\varepsilon, 0}-w^{0}-\mathcal{B}^{0}\left(0, \frac{z}{\varepsilon}\right)\left(q^{0}(y)\right)-\mathcal{B}^{1}\left(0, \frac{1-z}{\varepsilon}\right)\left(q^{0}(y)\right)\right\|_{m} \leq c \varepsilon^{\alpha} \\
\varepsilon\left\|\nabla\left(u^{\varepsilon, 0}-w^{0}-\mathcal{B}^{0}\left(0, \frac{z}{\varepsilon}\right)\left(q^{0}(y)\right)-\mathcal{B}^{1}\left(0, \frac{1-z}{\varepsilon}\right)\left(q^{0}(y)\right)\right)\right\|_{m} \leq c \varepsilon^{\alpha}
\end{array}\right.
$$

for some $m \geq 2,3 / 4<\alpha \leq 1$ and some constant $c>0$. Let $w^{\mathrm{int}}(t)$ be the solution of (9), we assume that $\mathbf{( H )}$ holds. Then, there exists $\varepsilon_{0}$ such that for all $0<\varepsilon<\varepsilon_{0}$, the system (1) has a unique weak solution $u^{\varepsilon} \in L^{\infty}\left(0, T ; L^{2}(\Omega)\right) \cap L^{2}\left(0, T ; H_{0}^{1}(\Omega)\right)$ with initial value $u^{\varepsilon, 0}$. Moreover,

(20)

$$
\left\|u^{\varepsilon}-\mathcal{L}\left(\frac{t}{\varepsilon}\right) w^{\mathrm{int}}-\mathcal{B}^{0}\left(\frac{t}{\varepsilon}, \frac{z}{\varepsilon}\right)(q(t, y))-\mathcal{B}^{1}\left(\frac{t}{\varepsilon} \frac{1-z}{\varepsilon}\right)(q(t, y))\right\|_{L^{\infty}\left(0, T ; L^{2}(\Omega)\right)} \leq C_{T} \varepsilon^{\alpha} .
$$

In addition, if $\alpha=3 / 4$ in (19), there exists a time $T_{0}$ which depends on $w^{0}$ and on $c$ (but not on $\varepsilon)$ such that (20) holds on $\left(0, T_{0}\right)$.

Let us give a few remarks about this theorem.

Remark 3. - 1) First, we note that when $3 / 4<\alpha \leq 1$, the uniform time of existence and convergence $T$ is only limited by the stability assumption $\left(b(t, \bar{k}) e^{i k \cdot y}\right)_{\bar{k} \in \mathbb{Z}_{a}^{3}} \in \mathcal{K}_{0}$, in particular, it may be arbitrarily large even if the data is large. This is due to the regularity of the limit system which is better than the regularity of the $3 D$ Navier-Stokes in the nonresonant case (see [2]).

2) We also point out that the assumption that the torus is non-resonant is used to ensure that $b(t, \bar{k})=0$ for $\bar{k}=\left(0,0, k_{3}\right)$ and that the result holds if we know that $b(t, \bar{k})=0$ for $\bar{k}=\left(0,0, k_{3}\right)$ as well as an estimate of the type (12). Indeed, for the modes $\bar{k}=\left(0,0, k_{3}\right)$, the boundary layer we get is of Prandtl type (the rotation does not play any part). It was handled in [23] only with the crucial assumption that the vertical viscosity over the horizontal one also goes to zero.

3) We shall see in the proof that the result is actually more precise. A sufficient condition on the regularity of $w^{0}$ is $s>d+5$ where $d$ is given in (12). The convergence will take place in a space with horizontal regularity $m$ where $2 \leq m \leq s-d-3$. Moreover, we notice that the error estimate, namely $C_{T} \varepsilon^{\alpha}$ sees the boundary layer and hence the boundary layer cannot be removed from the estimate (20). This is stronger than the estimates in [17, 23]. However, our result requires the use of an initial data which depends on $\varepsilon$ and which is sufficiently close to the approximate solution at $t=0$. The convergence is stated in $L^{\infty}\left(0, T ; L^{2}(\Omega)\right)$ but as will be seen from the proof in section 5.1, we need to prove estimates in a stronger 
space, namely $Y_{m}$. In particular, the solution we construct is a strong solution which yields the uniqueness of the weak solutions by the classical strong-weak uniqueness argument.

4) Finally, we note that the proof of Theorem 2 is completely different from the proof of the stability in the well-prepared case in [26] and the proof of the small data case in the illprepared case [23].

The paper is organized as follows: in the next section, we give some details about the construction of the approximate solution, then, in the next sections, we prove the linear stability of this approximate solution; finally section 5 is devoted to the proof of Theorem 2. Appendix $\mathrm{A}$ is devoted to the definition and the proof of some simple properties of semi-classical operator-valued pseudo-differential calculus. These properties are crucial in the study of the linear stability. Finally, the Appendix B gathers some useful properties of the Leray projection in the strip and in the half-space.

\section{Construction of an approximate solution}

\subsection{Some definitions and notations}

We will use the notations of [23]. Let us denote by $V^{0}$ the subspace of $L^{2}(\Omega)^{3}$ consisting of divergence-free vectors ( $\operatorname{div} \mathrm{u}=0)$, and tangent to $\partial \Omega\left(u_{3}(z=0)=u_{3}(z=h)=0\right)$

$$
V^{0}=\left\{u \in L^{2}(\Omega)^{3}, \nabla \cdot u=0, u_{3}(z=0)=u_{3}(z=h)=0\right\} ;
$$

we also define, for $m>0, V^{m}$ the space

$$
V^{m}=H^{m}(\Omega)^{3} \cap V^{0},
$$

where $H^{m}(\Omega)$ is the classical Sobolev space $W^{2, m}(\Omega)$.

Let $\left.\mathbb{T}_{a}^{3}=\mathbb{T}_{a}^{2} \times\right]-1,1\left[=\mathbb{T}_{a}^{2} \times \mathbb{R} / 2 \mathbb{Z}\right.$ be the torus of periods respectively $a_{1}, a_{2}$ and 2 , and $E$ the linear operator from $L^{2}(\Omega)^{3}$ into $L^{2}\left(\mathbb{T}_{a}^{3}\right)^{3}$ defined by $E u(z)=u(z)$ for $0<z<h$, and for $-h<z<0$

$$
E u(z)=S(u(-z)),
$$

where $S$ is the linear operator from $\mathbb{R}^{3}$ into $\mathbb{R}^{3}$, defined by

$$
S X_{1}=X_{1} \quad S X_{2}=X_{2} \quad S X_{3}=-X_{3}
$$

for all $X \in \mathbb{R}^{3}$, which corresponds to a symmetry with respect to the plan $X_{3}=0$.

We also introduce $V^{\prime m}=\left\{u \in H^{m}\left(\mathbb{T}_{a}^{3}\right)^{3}, \nabla \cdot u=0\right\}$, Hence $E^{\prime} E u=I d_{V^{0}}$ where $E^{\prime}$ is the restriction from $L^{2}\left(\mathbb{T}^{3}\right)^{3}$ onto $L^{2}(\Omega)^{3}$. In the sequel we will work in the space $V_{\mathrm{sym}}^{m}$,

$$
V_{\mathrm{sym}}^{m}=E^{\prime}\left(V^{\prime m} \cap E\left(V^{m}\right)\right)
$$

which consists of vectors $u \in V^{m}$ that satisfy extra boundary conditions on the vertical derivatives.

We also introduce a norm on $V_{\mathrm{sym}}^{m}$

$$
|u|_{V_{\mathrm{sym}}^{m}}^{2}=\frac{1}{2}|E u|_{V^{\prime m}}^{2}
$$

which is conserved by the group $\mathcal{L}$, namely $|\mathcal{L}(\tau) u|_{V_{\mathrm{sym}}^{m}}=|u|_{V_{\mathrm{sym}}^{m}}$, for every $\tau \in \mathbb{R}$. We will also use the following notations (see the appendix 2.2 of [23] for a precise construction): 
- $X=\left(y_{1}, y_{2}, z\right)=(y, z)$;

- $N^{\bar{k}}(X)=M^{\bar{k}}(z) e^{i k \cdot y}$ is an eigenvector of $L$ associated to the eigenvalue $i \lambda(\bar{k})=$ $i k_{3} /|k|$

- for $w \in L^{\infty}\left(0, T, V_{\mathrm{sym}}^{s}\right)$, we set

$$
w(t, X)=\sum_{\bar{k} \in \mathbb{Z}_{a}^{3}} b(t, \bar{k}) N^{\bar{k}}(X),
$$

then we have for all $t,|w(t)|_{V_{\text {sym }}^{s}}^{2}=2|\Omega| \sum_{\bar{k}}|b(t, \bar{k})|^{2}|\bar{k}|^{2 s}$.

\subsection{Study of the group}

We study here the group $\mathcal{L}$, in particular, we give the expression of the eigenvectors $N^{k}$. Using the construction in the appendix of [23], we get for $\bar{k}=\left(k, k_{3}\right) \in \mathbb{Z}_{a}^{3}$ and $k \neq(0,0)$ that $N^{\bar{k}}(X)=M^{\bar{k}}(z) e^{i k . y}$ where

$$
M^{\bar{k}}(z)=\left(\begin{array}{c}
2 \cos \left(k_{3} z\right) n_{1}(\bar{k}) \\
2 \cos \left(k_{3} z\right) n_{2}(\bar{k}) \\
2 i \sin \left(k_{3} z\right) n_{3}(\bar{k})
\end{array}\right)
$$

and

$$
\begin{aligned}
& n_{1}(\bar{k})=\frac{n_{3}(\bar{k})}{1-\lambda(\bar{k})^{2}}\left(i \frac{k_{2}}{|\bar{k}|}-\frac{k_{1}}{|\bar{k}|} \lambda(\bar{k})\right) \\
& n_{2}(\bar{k})=\frac{n_{3}(\bar{k})}{1-\lambda(\bar{k})^{2}}\left(-i \frac{k_{1}}{|\bar{k}|}-\frac{k_{2}}{|\bar{k}|} \lambda(\bar{k})\right) \\
& n_{3}(\bar{k})=\sqrt{\frac{1-\lambda(\bar{k})^{2}}{2}} .
\end{aligned}
$$

Notice then that $N^{\bar{k}}(X) \in V_{\text {sym }}^{s}$, and that we have $L N^{\bar{k}}=\lambda(\bar{k}) N^{\bar{k}}$.

\subsection{Approximate solution}

Here, we construct the approximate solution $U^{\text {app }}=\left(u^{\text {app }}, p^{\text {app }}\right)$. The aim is that $U^{\text {app }}$ satisfies (1) up to a small error and the boundary condition (2) exactly.

To guess a good choice for $U^{\text {app }}$, we expand the solution in the following form $U^{0}+\varepsilon U^{1}+$ $\varepsilon^{2} U^{2}+\ldots$ where

$$
U^{0}=\underline{U}^{0}(\tau, t, x)+\tilde{U}^{0}(\tau, t, y, Z)+\breve{U}^{0}\left(\tau, t, y, Z^{\prime}\right),
$$

where we recall that $\tau=t / \varepsilon, Z=z / \varepsilon$ and $Z^{\prime}=(1-z) / \varepsilon$. Even though it is not clear whether we can push this expansion to all order, this will allow us to guess the first terms. Hence, arguing as in [23], we get

$$
\underline{u}^{0}(\tau, t)=\mathcal{L}(\tau) \underline{u}^{0}(\tau=0, t)=\mathcal{L}(\tau) w^{\mathrm{int}}(t)
$$

which is exactly the term $u^{\text {int }}$ in (4).

We notice that $\underline{u}^{0}$ does not vanish on $\partial \Omega$; we only have $\left(\underline{u}^{0} \cdot n\right) / \partial \Omega$, where $n$ is the normal to the boundary. This requires the introduction of a boundary layer. For the boundary layer, we only construct $\tilde{U}^{0}$, near $z=0$, since the construction of $\breve{U}^{0}$ near $z=1$ is similar. We recall that the modes $\left(0, k_{3}\right)$ are excluded due to the assumption on the initial data and 
on the resonances of the torus. So, we only deal with the case $-1<\lambda(\bar{k})<1$. Using the construction in section 4 of [23], we get $\tilde{u}^{0}=\sum_{\bar{k} \in \mathbb{Z}_{a}^{3}} R^{\bar{k}}$ where

$$
\begin{aligned}
R^{\bar{k}}(z)= & -\frac{1}{2} b(t, k) \exp \left[i\left((k \cdot y)+\frac{\lambda(k) t}{\varepsilon}\right)\right] \\
& \times\left[h^{\bar{k},+} \exp \left(-\frac{(1+i)}{\sqrt{2}} \eta^{\bar{k},+} \frac{z}{\varepsilon}\right)+h^{\bar{k},-} \exp \left(-\frac{(1-i)}{\sqrt{2}} \eta^{\bar{k},-} \frac{z}{\varepsilon}\right)\right] .
\end{aligned}
$$

In a similar way, we get that $\breve{u}^{0}=\sum_{\bar{k} \in \mathbb{Z}_{a}^{3}} T^{\bar{k}}$ where $T^{k}$ has the same formula as $R^{k}$. Hence, $u^{b}=\tilde{u}^{0}+\breve{u}^{0}$.

It remains to construct the remainder term $u^{r}$. Of course, a good guess for $u^{r}$ is to take $\varepsilon U^{1}$. However, we would like to get an approximate solution $u^{\text {app }}$ which is divergence-free and which vanishes on the boundary. The rest term is under the form

$$
u^{r}=\sum_{\bar{k}}\left(R_{3}^{\bar{k}}+T_{3}^{\bar{k}}\right)+\sum_{\bar{k}} r^{\bar{k}}+\varepsilon \mathcal{Y}+\mathcal{R}^{3}
$$

and hence consists of four terms. We refer to [23] for the details on the construction of these four terms. The main property of this correction term is that for all $|\alpha|+|\beta| \leq s-d-5$,

$$
\left\|\left(\varphi(z) \partial_{z}\right)^{\beta} \partial_{y}^{\alpha} u^{r}\right\|_{L^{\infty}} \leq C_{\alpha} \varepsilon, \quad\left\|\partial_{z}\left(\varphi(z) \partial_{z}\right)^{\beta} \partial_{y}^{\alpha} u^{r}\right\|_{L^{\infty}} \leq C_{\alpha}
$$

where $\varphi(z)$ is a smooth bounded function which is equivalent to $z$ and $1-z$ in the vicinity of 0 and 1 respectively.

- $R_{3}^{\bar{k}}$ and $T_{3}^{\bar{k}}$ which are introduced to insure that $R^{\bar{k}}$ and $T^{\bar{k}}$ satisfy the divergence free condition. However, $R_{3}^{\bar{k}}$ creates a trace at $z=0$ and $T_{3}^{\bar{k}}$ creates a trace at $z=1$ of order $\varepsilon$.

- $r^{\bar{k}}$ which is used to cancel the traces $R_{3}^{\bar{k}}(z=0)$ and $T_{3}^{\bar{k}}(z=1)$. Since, we have to take $r^{\bar{k}}$ which is divergence-free, we have to construct $r_{1}^{\bar{k}}$ and $r_{2}^{\bar{k}}$ which have a trace at $z=0$ and $z=1$ of order $\varepsilon$. This is actually easier to handle than the trace on the third component we started with. Besides, the term $\frac{1}{\varepsilon} e \times r^{\bar{k}}$ is responsible for the Ekman damping in the limit equation.

- $\varepsilon \mathcal{Y}$ is introduced to cancel the non resonant oscillating terms which do not yield a contribution in the limit equation. More precisely, we have

$$
\mathcal{Y}(\tau, t)=-\mathcal{L}(\tau) \int_{0}^{\tau}\left[\mathcal{L}\left(-\tau^{\prime}\right) Q(w, w)-\bar{Q}(w, w)\right](t) d \tau^{\prime} .
$$

We also point out that due to the non resonance assumption, we know that $\|\mathcal{Y}(\tau, t)\|_{H^{s-d-1}} \leq C$ for a constant which does not depend on $\tau$ or $t<T$.

- $\mathcal{R}^{3}$ which takes into account the boundary condition of $r, \mathcal{Y}$. This was constructed in section 4.3 of [23].

\section{Linear stability}

We study the linearized system about the approximate solution:

$$
\mathcal{T}^{\varepsilon}(v, p)=F, \quad \nabla \cdot v=0, \quad x \in \mathbb{T}_{a}^{2} \times(0,1)
$$

where

$$
\mathcal{T}^{\varepsilon}(v, p)=\partial_{t} v+u^{\mathrm{app}} \cdot \nabla v+v \cdot \nabla u^{\mathrm{app}}+\nabla p+\frac{e \times v}{\varepsilon}-\varepsilon \Delta v
$$


with initial data

$$
v(0, x)=v_{0}(x)
$$

and the boundary condition (2). Let us define $v^{H F}$ by $\mathcal{F}_{y} v^{H F}=\mathbf{1}_{\varepsilon|k| \geq r} \mathcal{F}_{y} v$, for some $r>0$ and $\kappa^{s}\left(\varepsilon D_{y}\right) v$ by $\mathcal{F}_{y}\left(\kappa^{s} v\right)=\kappa^{s}(\varepsilon \xi) \mathcal{F}_{y} v(\xi)$ with $\kappa^{s}(\xi)$ a smooth function which vanishes for $|\xi| \geq 2 r$. The main result of this section is

Theorem 4. - We assume that $\mathbf{( H )}$ holds. Then, there exist $\gamma_{0}>0, \gamma>0$ such that for every $\varepsilon>0, T>0$, with $\varepsilon e^{\gamma_{0} T} \leq 1$, we have

$$
\begin{aligned}
\|v(T)\|^{2}+\varepsilon^{2} \| \nabla v^{H F} & (T)\left\|^{2}+\int_{0}^{T} \varepsilon\right\| \nabla v \|^{2} \\
& \lesssim e^{\gamma t}\left(\left\|v_{0}\right\|^{2}+\left\|v_{0}^{H F}\right\|_{1, \varepsilon}^{2}+\int_{0}^{T}\left|\left(\kappa^{s} F, \kappa^{s} v\right)\right|+\varepsilon \int_{0}^{T}\|F\|^{2}\right) .
\end{aligned}
$$

Throughout the paper, $\lesssim$ stands for $\leq C$ where $C>0$ is independent of $\varepsilon \in(0,1), T$ if $\varepsilon e^{\gamma_{0} T} \leq 1$. Note that by using the Cauchy Schwarz inequality and the Gronwall inequality, we can get from (30) the estimate

$$
\begin{aligned}
\|v(T)\|^{2}+\varepsilon^{2}\left\|\nabla v^{H F}(T)\right\|^{2}+\int_{0}^{T}\left(\varepsilon\|\nabla v\|^{2}+\varepsilon^{-1}\left\|v^{H F}\right\|_{1, \varepsilon}^{2}\right) & \\
& \lesssim e^{\tilde{\gamma} t}\left(\left\|v_{0}\right\|^{2}+\left\|v_{0}^{H F}\right\|_{1, \varepsilon}^{2}+\int_{0}^{T}(1+\varepsilon)\|F\|^{2}\right)
\end{aligned}
$$

for some $\tilde{\gamma}>\gamma$ which gives an estimate of $v$ with respect to the source term $F$ and the initial data only. Nevertheless, in order to handle the nonlinear stability, it is important to keep the term

$$
\int_{0}^{T}\left|\left(\kappa^{s} F, \kappa^{s} v\right)\right|
$$

in the right-hand side since it will allow to use the structure of the nonlinear term of the Navier-Stokes equation and hence to get some better estimates.

The aim of the remaining part of the section is to prove Theorem 4. Note that for the moment we have a control of the $L^{\infty}\left(0, T, H^{1}\right)$ norm only for the high frequency part of $v$. We will derive an estimate for all the frequencies in paragraph 3.6.

\subsection{Proof of Theorem 4}

We start with a localization in frequency of the equation similar to the well prepared case [26]. We will deal with large, medium and small frequencies in different ways. For a smooth bounded function $\kappa$, we apply the Fourier multiplier $\kappa\left(\varepsilon D_{y}\right)$ to the equation 13 . We get

$$
\mathcal{T}^{\varepsilon}(\kappa v, \kappa p)=\kappa F+\mathcal{C}
$$

where the commutator $\mathcal{C}$ is defined as

$$
\mathcal{C}=-\left[\kappa, u^{\mathrm{app}} \cdot \nabla\right] v-\left[\kappa, D u^{\mathrm{app}}\right] v .
$$

By using the same argument as in [26], [28], we have the estimate

$$
\|\mathcal{C}\|^{2} \lesssim \varepsilon^{2}\|\nabla v\|^{2}+\|v\|^{2} .
$$

$4{ }^{\mathrm{e}}$ SÉRIE - TOME $41-2008-\mathrm{N}^{\mathrm{o}} 6$ 
Note that these commutator estimates are actually proven in a more general case in Lemma 16.

We first deal with the case where $\kappa=\kappa^{L}$ is supported in $\varepsilon|k| \geq R$. We have

Proposition 5. - There exists $R>0$ sufficiently large such that we have for every $\varepsilon \in$ $(0,1)$ and every $T>0$ :

$$
\left\|\kappa^{L} v(T)\right\|_{1, \varepsilon}^{2}+\varepsilon^{-1} \int_{0}^{T}\left\|\kappa^{L} v\right\|_{1, \varepsilon}^{2} \lesssim \int_{0}^{T}\left(\|v\|_{1, \varepsilon}^{2}+\varepsilon\|F\|^{2}\right)+\left\|\kappa^{L} v_{0}\right\|_{1, \varepsilon}^{2} .
$$

Note that for the proof of this lemma we do not need to use (H).

Proof. - We use the same argument as in the well-prepared case treated in [26]. Using that $u^{\text {app }}$ is divergence free, the standard energy estimate for (31) gives

$$
\begin{aligned}
\left\|\kappa^{L} v(T)\right\|^{2}+\int_{0}^{T} \varepsilon\left\|\nabla \kappa^{L} v\right\|^{2} & \lesssim\left\|\kappa^{L} v_{0}\right\|^{2}+\int_{0}^{T}\left(\varepsilon^{-1}\left\|\kappa^{L} v\right\|^{2}+\|F\|\left\|\kappa^{L} v\right\|+\|\mathcal{C}\|^{2}\right) \\
& \lesssim\left\|\kappa^{L} v_{0}\right\|^{2}+\int_{0}^{T}\left(\varepsilon^{-1}\left\|\kappa^{L} v\right\|^{2}+\varepsilon\|F\|^{2}+\|\mathcal{C}\|^{2}\right) .
\end{aligned}
$$

We notice that

$$
\left\|\nabla \kappa^{L} v\right\|^{2} \geq R^{2} \varepsilon^{-1}\left\|\kappa^{L} v\right\|^{2}
$$

so that for $R$ sufficiently large, the singular term $\varepsilon^{-1}\left\|\kappa^{L} v\right\|^{2}$ in the right hand side of (33) can be absorbed by the left hand side. By using also (32), this yields

$$
\left\|\kappa^{L} v(T)\right\|^{2}+\varepsilon^{-1} \int_{0}^{T}\left\|\kappa^{L} v\right\|_{1, \varepsilon}^{2} \lesssim \int_{0}^{T}\left(\|v\|_{1, \varepsilon}^{2}+\varepsilon\|F\|^{2}\right)+\left\|\kappa^{L} v_{0}\right\|^{2} .
$$

To conclude, it suffices to estimate $\varepsilon^{2}\left\|\nabla \kappa^{L} v(T)\right\|^{2}$. This is an easy consequence of the following lemma:

Lemma 6. - Consider u a solution of

$$
\partial_{t} u-\varepsilon \Delta u+\frac{e \times u}{\varepsilon}+\nabla p=H, \quad \nabla \cdot u=0, \quad x \in \Omega
$$

with the initial condition $u(0, x)=u_{0}(x)$ and the boundary condition (2). Then, we have the estimate

(35) $\varepsilon^{2}\|\nabla u(T)\|^{2}+\int_{0}^{T}\left(\varepsilon^{3}\left\|\nabla^{2} u\right\|^{2}+\varepsilon\left\|\partial_{t} u\right\|^{2}\right) \lesssim \varepsilon^{2}\left\|\nabla u_{0}\right\|^{2}+\int_{0}^{T}\left(\varepsilon^{-1}\|u\|^{2}+\varepsilon\|H\|^{2}\right)$.

We first end the proof of Proposition 5 by using Lemma 6. We can use Lemma 6 with $u=\kappa^{L} v$ and

$$
H=-u^{\text {app }} \cdot \nabla \kappa^{L} v-\left(\kappa^{L} v\right) \cdot \nabla u^{\text {app }}+\kappa^{L} F+\mathcal{C} .
$$

This yields

$$
\varepsilon^{2}\left\|\nabla \kappa^{L} v\right\|^{2} \lesssim \varepsilon^{2}\left\|\nabla \kappa^{L} v_{0}\right\|^{2}+\int_{0}^{T}\left(\varepsilon^{-1}\left\|\kappa^{L} v\right\|^{2}+\varepsilon\left\|\nabla \kappa^{L} v\right\|^{2}+\varepsilon\|F\|^{2}+\|v\|_{1, \varepsilon}^{2}\right) .
$$

To conclude, it suffices to add (33) times a sufficiently large constant and the last estimate.

We now turn to the Proof of Lemma 6: 
Proof of Lemma 6. - We take the scalar product of (34) by $\partial_{t} u$, since $\partial_{t} u$ is divergence free and verifies the boundary condition (2); we have

$$
\left(\nabla p, \partial_{t} u\right)=0, \quad\left(-\Delta u, \partial_{t} u\right)=\frac{d}{d t}\left(\frac{1}{2}\|\nabla u\|^{2}\right)
$$

and hence we get

$$
\varepsilon\|\nabla u(T)\|^{2}+\int_{0}^{T}\left\|\partial_{t} u\right\|^{2} \lesssim \varepsilon\left\|\nabla u_{0}\right\|^{2}+\int_{0}^{T}\left(\|H\|+\varepsilon^{-1}\|u\|\right)\left\|\partial_{t} u\right\| .
$$

By using the Young inequality, we find after multiplication by $\varepsilon$

$$
\varepsilon^{2}\|\nabla u(T)\|^{2}+\int_{0}^{T} \varepsilon\left\|\partial_{t} u\right\|^{2} \lesssim \varepsilon^{2}\left\|\nabla u_{0}\right\|^{2}+\int_{0}^{T}\left(\varepsilon\|H\|^{2}+\varepsilon^{-1}\|u\|^{2}\right) .
$$

Next, we use the classical regularity result for the Stokes equation [11]. We consider (34) as

$$
-\varepsilon \Delta u+\nabla p=H-\frac{e \times u}{\varepsilon}-\partial_{t} u, \quad \nabla \cdot u=0
$$

and we find that

$$
\varepsilon^{2}\left\|\nabla^{2} u\right\|^{2} \lesssim\|H\|^{2}+\varepsilon^{-2}\|u\|^{2}+\left\|\partial_{t} u\right\|^{2} .
$$

To end the proof, it suffices to integrate in time, to multiply by $\varepsilon$ the last estimate and to use (36). This ends the proof of Lemma 6.

We now consider $R$ as fixed. The next step is to consider the case where $\kappa=\kappa^{s}$ is supported in $\varepsilon|k| \leq r$.

Proposition 7. - There exists $r>0$ sufficiently small such that we have for every $\varepsilon \in$ $(0,1)$ and every $T>0$

$$
\left\|\kappa^{s} v(T)\right\|^{2}+\int_{0}^{T} \varepsilon\left\|\kappa^{s} \nabla v\right\|^{2} \lesssim \int_{0}^{T}\left(\|v\|_{1, \varepsilon}^{2}+\left|\left(\kappa^{s} F, \kappa^{s} v\right)\right|\right)+\left\|\kappa^{s} v_{0}\right\|^{2} .
$$

Again, note that the assumption $(\mathbf{H})$ is not used.

Proof. - Again we use a direct energy estimate as in [26]. Since $\left(e \times \kappa^{s} v, \kappa^{s} v\right)=0$, we get

$$
\left\|\kappa^{s} v(T)\right\|^{2}+\int_{0}^{T} \varepsilon\left\|\kappa^{s} \nabla v\right\|^{2} \lesssim \int_{0}^{T}\left(\|\mathcal{C}\|^{2}+\left\|\kappa^{s} v\right\|^{2}+\left|\left(\kappa^{s} F, \kappa^{s} v\right)\right|+S^{\varepsilon}\right)+\left\|\kappa^{s} v_{0}\right\|^{2}
$$

where the singular term $S^{\varepsilon}$ is defined by

$$
S^{\varepsilon}=\varepsilon^{-1} \int_{\Omega}\left|\kappa^{s} v_{3}\right|\left(\left|\partial_{Z} u^{b}\right|+\left|\partial_{Z^{\prime}} u^{b}\right|\right)\left|\kappa^{s} v\right| d x
$$

Again, we use (32) to estimate $\mathcal{C}$ and hence it remains to study $S^{\varepsilon}$. To estimate this term, we shall use the inequality

$$
|f(t, x)|^{2} \leq\left(\int_{0}^{z}\left|\partial_{z} f\right|\right)^{2} \leq z \int_{0}^{z}\left|\partial_{z} f\right|^{2} \leq z \int_{0}^{1}\left|\partial_{z} f\right|^{2} \quad \text { if } \quad f_{/ z=0}=0 .
$$


Note that since $\partial_{z} \kappa^{s} v_{3}=-\partial_{1} \kappa^{s} v_{1}-\partial_{2} \kappa^{s} v_{2}$ because of the incompressiblity condition, we can use (38) twice to get

$$
\begin{aligned}
\left|\kappa^{s} v_{3}\right|^{2} & \leq z \int_{0}^{z}\left|\partial_{z} \kappa^{s} v_{3}\right|^{2} \lesssim z \int_{0}^{z}\left|\nabla_{h} \kappa^{s} v_{h}\right|^{2} \lesssim z^{3} \int_{0}^{1}\left|\partial_{z} \nabla_{h} \kappa^{s} v_{h}\right|^{2} \\
\left|\kappa^{s} v\right|^{2} & \lesssim z \int_{0}^{1}\left|\partial_{z} \kappa^{s} v\right|^{2}
\end{aligned}
$$

This yields

$$
\begin{aligned}
\varepsilon^{-1} \int_{\Omega}\left|\kappa^{s} v_{3}\right| & \left|\partial_{Z} u^{b, 0}\right|\left|\kappa^{s} v\right| d x \\
& \lesssim \varepsilon^{2}\left\|\partial_{z} \nabla_{h} \kappa^{s} v\right\|\left\|\partial_{z} \kappa^{s} v\right\| \sup _{y} \int_{0}^{+\infty} Z^{2}\left|\partial_{Z} \mathcal{B}^{0}(\tau, Z) w^{\text {int }}(t, y, 0)\right| d Z \\
& \lesssim \varepsilon r\left\|\nabla \kappa^{s} v\right\|^{2} .
\end{aligned}
$$

To get the last inequality, we have used that $\varepsilon\left\|\nabla_{h} \kappa^{s} f\right\| \leq r\left\|\kappa^{s} f\right\|$ by definition of $\kappa^{s}$ and our regularity assumption which gives

$$
\sup _{y} \int_{0}^{+\infty} Z^{2}\left|\partial_{Z} \mathcal{B}^{0}(\tau, Z) w^{\text {int }}(t, y, 0)\right| d Z<+\infty
$$

following (8). The same argument in the vicinity of the boundary $z=1$ shows that

$$
\varepsilon^{-1} \int_{\Omega}\left|\kappa^{s} v_{3}\right|\left|\partial_{Z^{\prime}} u^{b, 1}\right|\left|\kappa^{s} v\right| d x \lesssim \varepsilon r\left\|\nabla \kappa^{s} v\right\|^{2} .
$$

Consequently, we can choose $r$ sufficiently small to absorb the singular term $S^{\varepsilon}$ in the left hand side of (37). This ends the proof of Proposition 7.

Finally, it remains the most difficult case where $\kappa(\varepsilon k)=\kappa^{l}$ is supported in $r / 2 \leq \varepsilon|k| \leq$ $2 R$. Note that $r$ and $R$ are now fixed. We have the following estimate:

Proposition 8. - Under the assumptions of Theorem 4, we have for $\varepsilon$ and $T$ such that $\varepsilon e^{\gamma_{0} T} \leq 1$

$$
\left\|\kappa^{l} v(T)\right\|_{1, \varepsilon}^{2}+\int_{0}^{T} \varepsilon^{-1}\left\|\kappa^{l} v\right\|_{1, \varepsilon}^{2} \lesssim e^{\gamma_{0} T}\left\|\kappa^{l} v_{0}\right\|_{1, \varepsilon}^{2}+\int_{0}^{T}\left(\varepsilon e^{\gamma_{0} T}\|F\|^{2}+\|v\|_{1, \varepsilon}^{2}\right) .
$$

The assumption $\left(b(t, \bar{k}) e^{i k \cdot y}\right)_{\bar{k} \in \mathbb{Z}_{a}^{3}} \in \mathcal{K}$ is crucial in the proof of Proposition 8 .

\subsection{Proof of Proposition 8}

In this section, due to the oscillations in the boundary layers, we use an approach completely different from the one of the well-prepared case used in [26]. The proof of this Proposition is the most technical part and we split it into various steps. First, we rewrite (31) by using the Leray projection $\mathbb{P}\left(D_{y}\right)$ which is recalled in section B.2. Let us set $v^{l}=\kappa^{l} v$, we get the equation

$$
\partial_{t} v^{l}=\mathbb{P}\left(D_{y}\right) \bar{\kappa}^{l}\left(\varepsilon D_{y}\right) \mathbb{L}^{\varepsilon} v^{l}+\mathbb{P}\left(D_{y}\right) \bar{\kappa}^{l}\left(\kappa^{l} F+\kappa^{l} \mathcal{C}\right), \quad \nabla \cdot v^{l}=0, \quad x \in \Omega,
$$

where $\bar{\kappa}^{l}$ is compactly supported with a support slightly bigger than $\kappa^{l}$ and takes the value 1 on the support of $\kappa^{l}$ in order that $\bar{\kappa}^{l} \kappa=\kappa$ and $\mathbb{L}^{\varepsilon}$ is defined by

$$
\mathbb{L}^{\varepsilon} v=\varepsilon \Delta v-u^{\mathrm{app}} \cdot \nabla v-v \cdot \nabla u^{\mathrm{app}}-\frac{e \times v}{\varepsilon} .
$$


Next, we shall estimate differently $v^{l}$ in the interior of the domain and in the vicinity of the boundary. We decompose $v^{l}$ as

$$
v^{l}=\chi^{b}\left(\frac{z}{\delta}\right) v^{l}+\chi^{\mathrm{int}}(z) v^{l}+\chi^{b}\left(\frac{1-z}{\delta}\right) v^{l}
$$

where $\chi^{b}$ is compactly supported in $[0,2]$, and $\chi^{\mathrm{int}}$ is compactly supported in $[\delta, 1-\delta]$. Note that $\chi^{\text {int }}$ depends on $\delta$ though we forget this dependence in the notation. Multiplying (39) by $\chi$ for $\chi$ one of the truncation functions $\chi^{\text {int }}, \chi^{b, 0}=\chi^{b}(z / \delta), \chi^{b, 1}=\chi^{b}(1-z / \delta)$, we get

$$
\begin{aligned}
& \partial_{t}\left(\chi v^{l}\right)=\mathbb{P}\left(D_{y}\right) \bar{\kappa}^{l}\left(\varepsilon D_{y}\right) \mathbb{L}^{\varepsilon}\left(\chi v^{l}\right)+H \\
& \nabla \cdot\left(\chi v^{l}\right)=-\partial_{z} \chi v_{3}^{l}
\end{aligned}
$$

where

$$
H=\chi \mathbb{P}\left(D_{y}\right) \bar{\kappa}^{l}\left(\kappa^{l} F+\kappa^{l} \mathcal{C}\right)+\mathcal{C}^{1}+\mathcal{C}^{2}
$$

with the new commutators $\mathcal{C}^{1}$ and $\mathcal{C}^{2}$ defined by

$$
\begin{aligned}
& \mathcal{C}^{1}=\left[\chi, \mathbb{P}\left(D_{y}\right) \bar{\kappa}^{l}\left(\varepsilon D_{y}\right)\right] \mathbb{L}^{\varepsilon} v^{l} \\
& \mathcal{C}^{2}=\mathbb{P}\left(D_{y}\right) \bar{\kappa}^{l}\left(\varepsilon D_{y}\right)\left[\chi, \mathbb{L}^{\varepsilon}\right] v^{l} .
\end{aligned}
$$

Thanks to (155) in Lemma 19, we get that

$$
\left\|\mathcal{C}^{1}\right\| \lesssim\left\|v^{l}\right\|+\varepsilon\left\|\nabla v^{l}\right\|+\varepsilon^{2}\left\|\Delta v^{l}\right\| \lesssim\left\|v^{l}\right\|_{2, \varepsilon} .
$$

Note that in the following $\lesssim$ stands for $\leq C$ and that $C$ may depend on $\delta$. Besides, the explicit computation of $\mathcal{C}^{2}$ and a new use of the commutator estimates (see the appendix of [26] or Lemma 16) gives that

$$
\left\|\mathcal{C}^{2}\right\| \lesssim\left\|v^{l}\right\|+\varepsilon\left\|\nabla v^{l}\right\| \lesssim\left\|v^{l}\right\|_{1, \varepsilon} .
$$

Finally, note that if we choose $\overline{\bar{\kappa}}^{l}$ such that the support of $\overline{\bar{\kappa}}^{l}$ is again slightly larger than the one of $\bar{\kappa}^{l}$ then we have

$$
H=\overline{\bar{\kappa}}^{l}\left(\varepsilon D_{y}\right) H .
$$

3.2.1. Interior estimates. - We start with the case $\chi=\chi^{\text {int }}$. The estimate of $\chi^{\text {int }} v^{l}$ can be obtained by a direct energy estimate. We shall first establish the estimate:

$$
\left\|\chi^{\text {int }} v^{l}(T)\right\|^{2}+\varepsilon^{-1} \int_{0}^{T}\left\|\chi^{\text {int }} v^{l}\right\|_{1, \varepsilon}^{2} \lesssim\left\|\chi^{\text {int }} v_{0}^{l}\right\|^{2}+\int_{0}^{T}\left(\varepsilon\left\|v^{l}\right\|_{2, \varepsilon}^{2}+\varepsilon\|F\|^{2}+\|v\|_{1, \varepsilon}^{2}\right) .
$$

The only difficulty is that we have to deal with the fact that $\chi^{\text {int }} v^{l}$ is not divergence free. When we take the scalar product of (41) by $\chi^{\text {int }} v^{l}$, we can write

$$
\begin{aligned}
\left(\mathbb{P}\left(D_{y}\right) \bar{\kappa}^{l}\left(\varepsilon D_{y}\right) \mathbb{L}^{\varepsilon}\left(\chi^{\mathrm{int}} v^{l}\right), \chi^{\mathrm{int}} v^{l}\right)=\left(\mathbb{L}^{\varepsilon}\left(\chi^{\mathrm{int}} v^{l}\right), \mathbb{P}\left(D_{y}\right) \bar{\kappa}^{l}\left(\varepsilon D_{y}\right)\left(\chi^{\mathrm{int}} v^{l}\right)\right) \\
=\left(\mathbb{L}^{\varepsilon}\left(\chi^{\mathrm{int}} v^{l}\right), \chi^{\mathrm{int}} v^{l}\right)+\left(\mathbb{L}^{\varepsilon}\left(\chi^{\mathrm{int}} v^{l}\right),\left[\mathbb{P}\left(D_{y}\right) \bar{\kappa}^{l}\left(\varepsilon D_{y}\right), \chi^{\mathrm{int}}\right] v^{l}\right)
\end{aligned}
$$


since $v^{l}$ is divergence free. Hence by using again the commutator estimate (155) of Lemma 19 , we get

$$
\begin{aligned}
& \left(\mathbb{P}\left(D_{y}\right) \bar{\kappa}^{l}\left(\varepsilon D_{y}\right) \mathbb{L}^{\varepsilon}\left(\chi^{\text {int }} v^{l}\right), \chi^{\text {int }} v^{l}\right)= \\
& \quad\left(\mathbb{L}^{\varepsilon}\left(\chi^{\text {int }} v^{l}\right), \chi^{\text {int }} v^{l}\right)+\mathcal{O}(1)\left(\left\|v^{l}\right\|+\varepsilon\left\|\nabla v^{l}\right\|+\varepsilon^{2}\left\|\Delta v^{l}\right\|\right)\left\|\chi^{\text {int }} v^{l}\right\| .
\end{aligned}
$$

where $\mathcal{O}(1)$ is bounded by a constant which is independent of $\varepsilon$. The first term in the above equality can be handled by standard integration by parts as previously. This yields

$$
\begin{aligned}
& \left\|\chi^{\mathrm{int}} v^{l}(T)\right\|^{2}+\int_{0}^{T} \varepsilon\left\|\nabla\left(\chi^{\mathrm{int}} v^{l}\right)\right\|^{2} \\
& \lesssim\left\|\chi^{\mathrm{int}} v_{0}^{l}\right\|^{2}+\int_{0}^{T}\left(\left(\left\|v^{l}\right\|_{2, \varepsilon}+\|H\|\right)\left\|\chi^{\mathrm{int}} v^{l}\right\|+S^{\varepsilon}\right)
\end{aligned}
$$

where the singular term $S^{\varepsilon}$ is given by

$$
S^{\varepsilon}=\varepsilon^{-1}\left|\left(\chi^{\mathrm{int}} \partial_{Z} u^{b} v^{l}, \chi^{\mathrm{int}} v^{l}\right)\right|+\varepsilon^{-1}\left|\left(\chi^{\mathrm{int}} \partial_{Z^{\prime}} u^{b} v^{l}, \chi^{\mathrm{int}} v^{l}\right)\right|:=S_{1}^{\varepsilon}+S_{2}^{\varepsilon} .
$$

By using the localization of the support of $\chi^{\text {int }}$ we have

$$
\begin{aligned}
\left|S_{1}^{\varepsilon}\right| & \lesssim \sum_{\mathbf{k}, \pm}\left|b(t, \mathbf{k})\left\|M^{\mathbf{k}}(0) \mid \varepsilon^{-1} \exp \left(-\frac{\eta^{\mathbf{k}, \pm} \delta}{\sqrt{2} \varepsilon}\right)\right\| \chi^{\text {int }} v^{l} \|^{2}\right. \\
& \lesssim \sum_{\mathbf{k}, \pm} \frac{|b(t, \mathbf{k})| M^{\mathbf{k}}(0)}{\eta^{\mathbf{k}, \pm}}\left\|\chi^{\text {int }} v^{l}\right\|^{2} \lesssim\left\|\chi^{\text {int }} v^{l}\right\|^{2}
\end{aligned}
$$

and hence this term is well controlled. The estimate of $S_{2}^{\varepsilon}$ is similar. To conclude, we finally notice that since the Fourier transform of $v^{l}$ is localized in $\varepsilon|k| \geq r$, we can write

$$
\begin{aligned}
\left\|\nabla\left(\chi^{\mathrm{int}} v^{l}\right)\right\|^{2} & =\| \nabla\left(\chi^{\mathrm{int}} \bar{\kappa}^{l}\left(\varepsilon \partial_{y}\right) v^{l}\left\|^{2}=\right\| \bar{\kappa} \nabla\left(\chi^{\mathrm{int}} v^{l}\right) \|^{2}\right. \\
& \geq \varepsilon^{-2}\left\|\chi^{\mathrm{int}} v^{l}\right\|^{2}
\end{aligned}
$$

and hence, we deduce (49) from (50) by using the Young inequality which gives for every $\eta>0$

$$
\left(\left\|v^{l}\right\|_{2, \varepsilon}+\|H\|\right)\left\|\chi^{\text {int }} v^{l}\right\| \leq C(\eta) \varepsilon\left(\|H\|^{2}+\left\|v^{l}\right\|_{2, \varepsilon}^{2}\right)+\eta \varepsilon^{-1}\left\|\chi^{\text {int }} v^{l}\right\|^{2}
$$

and the estimates (32), (46), (47).

3.2.2. Estimates near the boundary. - We now explain how to estimate $\chi^{b}(z / \delta) v^{l}$ which will be denoted as $\chi^{b} v^{l}$ in the following in order to simplify the notations. The estimate of $\chi^{b, 1} v^{l}$ which can be obtained in a similar way will not be detailled. We shall establish

$$
\begin{aligned}
\left\|\chi^{b} v^{l}(T)\right\|^{2}+ & \int_{0}^{T}\left(\varepsilon^{-1}\left\|\chi^{b} v^{l}\right\|^{2}+\varepsilon\left\|\nabla\left(\chi^{b} v^{l}\right)\right\|^{2}\right) \\
\lesssim & e^{\gamma_{0} T}\left\|v^{l}(0)\right\|_{1, \varepsilon}^{2}+\varepsilon e^{\gamma_{0} T} \int_{0}^{T}\|F\|^{2} \\
& +\varepsilon e^{\gamma_{0} T} \int_{0}^{T}\left(\|v\|^{2}+\varepsilon^{2}\|\nabla v\|^{2}+\varepsilon^{4}\left\|\Delta v^{l}\right\|^{2}+\varepsilon^{2}\left\|\partial_{t} v^{l}\right\|^{2}\right) .
\end{aligned}
$$

We study (41), (42) with $\chi=\chi^{b, 0}$. Note that since $\chi^{b} v^{l}$ is compactly supported in $\mathbb{T}_{a}^{2} \times[0,2 \delta]$, we can use Lemma 20 and replace the Leray projection $\mathbb{P}\left(D_{y}\right)$ by the Leray projection in the 
half-space $\mathbb{P}_{+}\left(D_{y}\right)$ modulo a small remainder term. This means that we can study in $\mathbb{T}_{a}^{2} \times \mathbb{R}_{+}$ the equation

$$
\partial_{t}\left(\chi^{b} v^{l}\right)=\mathbb{P}_{+}\left(D_{y}\right) \bar{\kappa}\left(\varepsilon D_{y}\right) \mathbb{L}^{\varepsilon}\left(\chi^{b} v^{l}\right)+H+E^{0}, \quad \nabla \cdot\left(\chi^{b} v^{l}\right)=-\partial_{z} \chi v^{l}
$$

where

$$
E^{0}=\bar{\chi}^{b}\left(\mathbb{P}\left(D_{y}\right)-\mathbb{P}_{+}\left(D_{y}\right)\right) \bar{\kappa}^{l}\left(\varepsilon D_{y}\right) \mathbb{L}^{\varepsilon}\left(\chi^{b} v^{l}\right)
$$

where $\bar{\chi}^{b}$ is a smooth function with a support slightly bigger than $\chi^{b}$. Thanks to Lemma 20, we have

$$
\left\|E^{0}\right\| \lesssim\left\|v^{l}\right\|+\varepsilon\left\|\nabla v^{l}\right\|+\varepsilon^{2}\left\|\Delta v^{l}\right\| \lesssim\left\|v^{l}\right\|_{2, \varepsilon} .
$$

Again, note that $E^{0}$ verifies

$$
E^{0}=\overline{\bar{\kappa}}^{l}\left(\varepsilon D_{y}\right) E^{0} .
$$

We add to (53) the only boundary condition

$$
\chi^{b} v^{l}(t, y, 0)=0 \text {. }
$$

Since $\chi^{b} v^{l}$ is not divergence free, we first lift the divergence to recover a problem with a divergence free constraint. We choose in a classical way $d$ such that

$$
\nabla \cdot d=-\partial_{z} \chi^{b} v_{3}^{l}, \quad d(t, y, 0)=0
$$

and also in such a way that

$$
\bar{\kappa}^{l}\left(\varepsilon D_{y}\right) d=d
$$

This is possible (see [11]) since

$$
\int \partial_{z} \chi^{b} v_{3}^{l}=-\int \chi^{b} \partial_{z} v_{3}^{l}=\int_{z} \chi^{b}(z) \int_{y} \nabla_{y} \cdot v_{h}^{l}=0 .
$$

Moreover, we can have

$$
\|d\|_{H^{s+1}} \lesssim\left\|v^{l}\right\|_{H^{s}}, \quad s \geq 0 .
$$

Note that since $d$ is chosen with the property (58), we can use that $r \lesssim \varepsilon|k|$ on the support of $\bar{\kappa}^{l}$ and (59) to get that

$$
\varepsilon^{-1}\|d\| \lesssim\left\|v^{l}\right\|
$$

Moreover, by taking the time derivative of (57), we also get that

$$
\varepsilon^{-1}\left\|\partial_{t} d\right\| \lesssim\left\|\partial_{t} v^{l}\right\|
$$

Now, let us set $w=\chi^{b} v^{l}-d$, we deduce from (53), (57) that $w$ solves

$$
\begin{aligned}
& \partial_{t} w=\mathbb{P}_{+}\left(D_{y}\right) \bar{\kappa}\left(\varepsilon D_{y}\right) \mathbb{L}^{b, \varepsilon} w+H+E^{0}+E^{1}+E^{2}, \\
& \nabla \cdot w=0
\end{aligned}
$$

for $x=(y, z) \in \mathbb{T}_{a}^{2} \times \mathbb{R}_{+}$, with the boundary condition

$$
w(t, y, 0)=0
$$

$4^{\mathrm{e}}$ SÉRIE - TOME $41-2008-\mathrm{N}^{\mathrm{o}} 6$ 
where $\mathbb{L}^{b, \varepsilon}$ is the operator

$$
\begin{aligned}
\mathbb{L}^{b, \varepsilon} w= & \varepsilon \Delta w \\
& -\left(u^{\mathrm{int}}\left(\frac{t}{\varepsilon}, t, y, 0\right)+u^{b, 0}\left(\frac{t}{\varepsilon}, \frac{z}{\varepsilon}, t, y\right)+\bar{\chi}\left(\frac{z}{\delta}\right)\left(u^{\mathrm{int}}\left(\frac{t}{\varepsilon}, t, y, z\right)-u^{\mathrm{int}}\left(\frac{t}{\varepsilon}, t, y, 0\right)\right)\right) \cdot \nabla w \\
& -w \cdot \nabla\left(u^{\mathrm{int}}\left(\frac{t}{\varepsilon}, t, y, 0\right)+u^{b, 0}\left(\frac{t}{\varepsilon}, \frac{z}{\varepsilon}, t, y\right)+\bar{\chi}\left(\frac{z}{\delta}\right)\left(u^{\mathrm{int}}\left(\frac{t}{\varepsilon}, t, y, z\right)-u^{\mathrm{int}}\left(\frac{t}{\varepsilon}, t, y, 0\right)\right)\right) \\
& -\frac{e \times w}{\varepsilon} .
\end{aligned}
$$

In this operator, we have introduced $\bar{\chi}$ which is again a smooth compactly supported function such that $\bar{\chi} \chi^{b}=\chi^{b}$. Moreover, we recall that the notation $u^{b, 0}$ refers to the main boundary layer in the vicinity of $z=0$. The main interest of the introduction of $\bar{\chi}$ is that the term

$$
w^{\delta}=\bar{\chi}\left(\frac{z}{\delta}\right)\left(u^{\text {int }}\left(\frac{t}{\varepsilon}, t, y, z\right)-u^{\text {int }}\left(\frac{t}{\varepsilon}, t, y, 0\right)\right)
$$

verifies

$$
\left\|w^{\delta}\right\|_{L^{\infty}} \lesssim \delta, \quad\left\|\nabla w^{\delta}\right\|_{L^{\infty}} \lesssim 1
$$

In the above estimates, $\lesssim$ is independent of $\delta$ for $0<\delta \leq 1$. In the right-hand side of (62), $E^{1}$ is the error term coming from $d$, i.e

$$
E^{1}=\partial_{t} d-\mathbb{P}_{+}\left(D_{y}\right) \bar{\kappa}\left(\varepsilon D_{y}\right) \mathbb{L}^{b, \varepsilon} d .
$$

Hence we have

$$
\left\|E^{1}\right\| \lesssim\left\|\partial_{t} d\right\|+\|\nabla d\|+\|d\|+\varepsilon\|\Delta d\|+\varepsilon^{-1}\|d\|
$$

and hence, thanks to (59), (60), (61), we get

$$
\left\|E^{1}\right\| \lesssim \varepsilon\left\|\partial_{t} v^{l}\right\|+\left\|v^{l}\right\|+\varepsilon\left\|\nabla v^{l}\right\| \lesssim\left\|\partial_{t} v^{l}\right\|+\left\|v^{l}\right\|_{1, \varepsilon} .
$$

The other error term $E^{2}$ is defined as

$$
\begin{array}{r}
E^{2}=-\mathbb{P}_{+}\left(\partial_{y}\right) \kappa\left(\varepsilon \partial_{y}\right)\left(u^{b, 1}\left(\frac{t}{\varepsilon}, \frac{1-z}{\varepsilon}, y, 0\right)+u^{r}\right) \cdot \nabla\left(\chi^{b} v^{l}\right) \\
\left.-\chi^{b} v^{l} \cdot \nabla\left(u^{b, 1}\left(\frac{t}{\varepsilon}, \frac{1-z}{\varepsilon}, t, y\right)+u^{r}\right)\right)
\end{array}
$$

so that by using (27) and the same trick as in (51) with the regularity assumption on the coefficients, we get

$$
\left\|E^{2}\right\| \lesssim \varepsilon\left\|\nabla v^{l}\right\|+\left\|v^{l}\right\| \lesssim\left\|v^{l}\right\|_{1, \varepsilon} .
$$

Finally, note that $E^{1}$ and $E^{2}$ verify

$$
\overline{\bar{\kappa}}^{l} E^{1}=E^{1}, \quad \overline{\bar{\kappa}}^{l} E^{2}=E^{2} .
$$

To estimate the solution of (62), (63), we can use the following general principle:

Lemma 9. - Consider a linear equation of parabolic type in a domain $\Omega$

$$
\partial_{t} w=A^{\varepsilon} w+F
$$

with the boundary condition $w_{/ \partial \Omega}=0$ and the initial condition $w(0, x)=w_{0}(x)$. Consider two weighted norms $N_{T}^{\varepsilon}$ and $\|\cdot\|_{T, \varepsilon}$. Assume that there exists an approximate solver $G^{\text {app }}$ such that if we define

$$
w^{\text {app }}(t)=G^{\text {app }} \mathcal{F}, \quad \mathcal{F}=\left(F, w_{0}\right)
$$


then $w^{\text {app }}$ satisfies the boundary condition and the initial condition and moreover, there exists $C_{T, \varepsilon}>0$ such that

$$
N_{T}^{\varepsilon}\left(w^{\mathrm{app}}\right) \leq C_{T, \varepsilon}\|\mathcal{F}\|_{T, \varepsilon}
$$

and if we define the rest operator $R^{\mathrm{app}}$ as

$$
R^{\text {app }} \mathcal{F}=\partial_{t} w^{\text {app }}-A^{\varepsilon} w^{\text {app }}-F,
$$

then, there exists $C_{T, \varepsilon}^{1}>0$ such that

$$
\left\|\left(R^{\mathrm{app}} \mathcal{F}, 0\right)\right\|_{T, \varepsilon} \leq C_{T, \varepsilon}^{1}\|\mathcal{F}\|_{T, \varepsilon} .
$$

Moreover, for every $\varepsilon>0$ and $T>0$ such that

$$
C_{T, \varepsilon}^{1}<1,
$$

there exists $C>0\left(C=\sum_{k \geq 0}\left(C_{T, \varepsilon}^{1}\right)^{k}\right)$ such that the exact solution of (69) satisfies

$$
N_{T}^{\varepsilon}(w) \leq C C_{T, \varepsilon}\|\mathcal{F}\|_{T, \varepsilon} .
$$

The proof of this lemma which only relies on a simple iteration scheme is postponed to the end of the section.

We shall first explain how we can use Lemma 9 to estimate the solution of (62), (63). In other words, we need to find an approximate solver $G^{\text {app }}$. A similar idea was used in [18]; nevertheless, here our approximate solver will be completely different. We define the dilation operators

$$
M_{\varepsilon} f(Z)=\sqrt{\varepsilon} f(\varepsilon Z), \mathcal{M}_{\varepsilon} f(\tau, Z)=\varepsilon f(\varepsilon \tau, \varepsilon Z) .
$$

Note that $M_{\varepsilon}$ is an isometry on $L^{2}\left(\mathbb{R}_{+}\right)$and $\mathcal{M}_{\varepsilon}$ from $L^{2}\left([0, T] \times \mathbb{R}_{+}\right)$to $L^{2}\left([0, T / \varepsilon] \times \mathbb{R}_{+}\right)$. We notice that thanks to (65), we can rewrite the operator as

$$
\begin{aligned}
& \mathbb{L}^{b, \varepsilon} w=\frac{1}{\varepsilon} M_{\varepsilon}^{-1} \mathbb{L}^{b}\left(q(t, y), \varepsilon D_{y}\right) M_{\varepsilon} w+\mathcal{O}(1)(\delta|\nabla w|+|w|) \\
& \mathbb{P}_{+}\left(D_{y}\right)=M_{\varepsilon}^{-1} \mathbb{P}_{+}\left(\varepsilon D_{y}\right) M_{\varepsilon}
\end{aligned}
$$

where $q(t, y)=\left(q_{\bar{k}}(t, y)\right)$ with

$$
q_{\bar{k}}(t, y)=b(t, \bar{k}) e^{i k \cdot y}
$$

In the above equality, $\mathcal{O}(1)$ is bounded by a number independent of $\delta$ if $\varepsilon / \delta \leq 1$. The rescaled operator $\mathbb{L}^{b}$ is defined by

$$
\begin{aligned}
\mathbb{L}^{b}(q, \xi) w= & \left(\partial_{Z Z}-|\xi|^{2}\right) w-\left(\mathcal{L}^{0} q+\mathcal{B}^{0}(\tau, Z) q\right) \cdot\left(\begin{array}{c}
i \xi \\
\partial_{z}
\end{array}\right) w \\
& -w \cdot\left(\begin{array}{c}
i \xi \\
\partial_{z}
\end{array}\right)\left(\mathcal{L}^{0} q+\mathcal{B}^{0}(\tau, Z) q\right)-e \times w
\end{aligned}
$$

Next, we use a frozen time approximation, we rewrite (62) as

$$
\frac{1}{\varepsilon} \mathcal{M}_{\varepsilon}^{-1} \mathbb{P}_{+}\left(\varepsilon D_{y}\right) \bar{\kappa}\left(\varepsilon D_{y}\right) \mathcal{T}\left(q(0, y), \varepsilon D_{y}\right) \mathcal{M}_{\varepsilon} w+\mathcal{R}^{\varepsilon} w=H^{1}
$$

where the symbol $\mathcal{T}(q, \xi)$ is the differential operator acting only on the $\tau$ and $Z$ variables defined by

$$
\mathcal{T}(q, \xi) w=\partial_{\tau} w-\mathbb{L}_{+}^{0}(q, \xi) w
$$


where $\mathbb{L}_{+}^{0}$ is defined in (16) and $\mathcal{R}^{\varepsilon} w$ is defined by

$$
\begin{aligned}
\mathcal{R}^{\varepsilon} w= & \frac{1}{\varepsilon} \mathcal{M}_{\varepsilon}^{-1} \mathbb{P}_{+}\left(\varepsilon D_{y}\right) \bar{\kappa}\left(\varepsilon D_{y}\right)\left(\mathcal{T}\left(q(0, y), \varepsilon D_{y}\right)-\mathcal{T}\left(q(t, y), \varepsilon D_{y}\right)\right) \mathcal{M}_{\varepsilon} w \\
& +\mathbb{P}_{+}\left(D_{y}\right) \mathcal{O}(1)(\delta|\nabla w|+|w|)
\end{aligned}
$$

and hence satisfies the estimate

$$
\int_{0}^{T}\left\|\mathcal{R}^{\varepsilon} w\right\|^{2} \lesssim\left(T^{2}+\delta^{2}\right) \int_{0}^{T}\|\nabla w\|^{2}+\varepsilon^{-2}\left(\delta^{2}+T^{2}\right) \int_{0}^{T}\|w\|^{2} .
$$

The source term $H^{1}$ in the right-hand side of (74) is defined by

$$
H^{1}=H+E^{0}+E^{1}+E^{2} .
$$

Thanks to our assumption $(\mathrm{H})$, it is natural to define our approximate solver $G^{\text {app }}$ and our approximate solution $w^{\text {app }}$ as

$$
w^{\text {app }}=G^{\text {app }} \mathcal{F}=\mathcal{M}_{\varepsilon}^{-1} \mathrm{Op}_{g} \overline{\bar{\kappa}}^{l}\left(\varepsilon D_{y}\right) \mathcal{M}_{\varepsilon} \mathcal{D}_{\varepsilon} \mathcal{F},
$$

where

$$
\mathcal{F}=\left(\begin{array}{c}
H^{1} \\
w_{0}
\end{array}\right), \quad \mathcal{D}_{\varepsilon} \mathcal{F}=\left(\varepsilon H^{1}, \varepsilon^{\frac{1}{2}} w_{0}\right),
$$

and with a slight abuse of notation, we define $\mathcal{M}_{\varepsilon} \mathcal{F}$ as

$$
\mathcal{M}_{\varepsilon} \mathcal{F}=\left(\mathcal{M}_{\varepsilon} H^{1}, M_{\varepsilon} w_{0}\right) .
$$

To define the operator-valued symbol $g$, we first define for data $\mathcal{F}(\tau, Z)=\left(F(\tau, Z), u_{0}(Z)\right)$, the operator $G(q, \xi)$ (acting on functions depending on $\tau$ and $Z$ ) such that $G(q, \xi) \mathcal{F}(\tau, Z):=$ $u(\tau, Z)$ is the solution of

$$
\partial_{\tau} u=\mathbb{P}_{+}(\xi) \mathbb{L}_{+}^{0}(\xi) u+F, \quad i \xi \cdot u_{h}+\partial_{Z} u_{3}=0
$$

such that $u(0, Z)=u_{0}(Z), u(\tau, 0)=0$.

By using the operator $G(q, \xi)$, we can define a symbol $g$ such that

$$
g(y, \xi)=G(q(0, y), \xi)
$$

and then a semi-classical operator-valued pseudo-differential operator $\mathrm{Op}_{g}$ as in the Appendix A. In particular for $\mathcal{F}(\tau, y, Z)$, we have the definition

$$
\mathrm{Op}_{g} \mathcal{F}=\sum_{k} e^{i k y} G(q(0, y), \varepsilon k) \hat{\mathcal{F}}(\tau, k, Z) .
$$

With a slight abuse of notation, we shall sometimes use the notation $G\left(q(0, y), \varepsilon D_{y}\right)$ in place of $\mathrm{Op}_{g}$. Note that the operator $\mathcal{M}_{\varepsilon}$ acts only on the $\tau$ and $Z$ variables. Consequently, we can write

$$
G^{\mathrm{app}} \mathcal{F}=\mathrm{Op}_{g^{\mathrm{app}}}, \quad g^{\mathrm{app}}(y, \xi)=\mathcal{M}_{\varepsilon}^{-1} g(y, \xi) \mathcal{M}_{\varepsilon} \mathcal{D}_{\varepsilon}
$$

and hence $G^{\text {app }}$ is itself an operator valued semi-classical pseudo-differential operator with symbol $g^{\text {app }}$.

Note that because of (48), (55), (68), since we want to solve (74) only for data such that

$$
\overline{\bar{\kappa}}^{l}\left(\varepsilon D_{y}\right) H^{1}=H^{1}, \quad \overline{\bar{\kappa}}^{l} w_{0}=w_{0},
$$


the introduction of $\overline{\bar{\kappa}}^{l}$ in the definition (77) is justified. To prove that $w^{\text {app }}=G^{\text {app }} \mathcal{F}$ is a good approximate solution, we want to use the semi-classical pseudo-differential calculus of section A seeing the symbol $g(y, \xi)$ as an operator on $L^{2}\left([0, T] \times \mathbb{R}_{+}\right)$or $L^{\infty}\left([0, T], L^{2}\left(\mathbb{R}_{+}\right)\right)$.

The aim of the following lemma is to study the dependence of $G(q, \xi)$ in $q$. In particular, we prove that it is smooth in $q$ and we estimate the derivatives. This will imply that $g$ is smooth in $y$ and hence we will be able to use Lemmas 16 and 17 of Appendix A.

We introduce the following notations:

$$
\begin{array}{cl}
|v|_{\Theta, 2}=\|v\|_{L^{2}\left((0, \Theta) \times \mathbb{R}_{+}\right)}, & |v|_{\Theta, \infty}=\|v\|_{L^{\infty}\left((0, \Theta), L^{2}\left(\mathbb{R}_{+}\right)\right)}, \\
\|v\|_{\Theta, 2}=\|v\|_{L^{2}\left((0, \Theta) \times \mathbb{T}_{a}^{2} \times \mathbb{R}_{+}\right)}, & \|v\|_{\Theta, \infty}=\|v\|_{L^{\infty}\left((0, \Theta), L^{2}\left(\mathbb{T}_{a}^{2} \times \mathbb{R}_{+}\right)\right)} .
\end{array}
$$

Lemma 10. - Thanks to $(H)$, for every $\xi$ in the support of $\overline{\bar{\kappa}}^{l}$, for every $q$ in $\mathcal{K}$ and for every $m$, there exist $\alpha_{m}>0$ and $C_{m}>0$ such that for every $\Theta>0$, for every $\mathcal{F}=\left(F(t, \cdot), u_{0}(\cdot)\right)$, $F(t, \cdot), u_{0}(\cdot) \in H_{\xi}$

$$
\begin{aligned}
& \left|D_{q}^{m} G(q, \xi) \mathcal{F}\right|_{\Theta, \infty}+\left|D_{q}^{m} G(q, \xi) \mathcal{F}\right|_{\Theta, 2}+\left|\partial_{Z} D_{q}^{m} G(q, \xi) \mathcal{F}\right|_{\Theta, 2} \\
& \quad \leq C_{m}\left(|F|_{\Theta, 2}+\left|u_{0}\right|_{L^{2}\left(\mathbb{R}_{+}\right)}\right) \\
& \quad\left|\partial_{Z} D_{q}^{m} G(q, \xi) \mathcal{F}\right|_{\Theta, \infty}+\left|\partial_{\tau} D_{q}^{m} G(q, \xi) \mathcal{F}\right|_{\Theta, 2}+\left|\partial_{Z Z} D_{q}^{m} G(q, \xi) \mathcal{F}\right|_{\Theta, 2} \\
& \quad \leq C_{m}\left(|F|_{\Theta, 2}+\left|u_{0}\right|_{H^{1}\left(\mathbb{R}_{+}\right)}\right) .
\end{aligned}
$$

We also postpone the proof of this Lemma to the end of the section.

Thanks to Lemma 10 and Lemma 15, we get that

$$
\begin{aligned}
\left\|w^{\mathrm{app}}\right\|_{T, 2}=\left\|G^{\mathrm{app}} \mathcal{F}\right\|_{T, 2} & \left.=\| \mathrm{Op}_{g} \overline{\bar{\kappa}}^{l}\left(\varepsilon \partial_{y}\right) \mathcal{M}_{\varepsilon} \mathcal{D}_{\varepsilon} \mathcal{F}\left(\tau^{\prime}, Z\right) d \tau^{\prime}\right) \|_{T / \varepsilon, 2} \\
& \lesssim \varepsilon\left\|\mathcal{M}^{\varepsilon} H^{1}\right\|_{T / \varepsilon, 2}+\sqrt{\varepsilon}\left\|M_{\varepsilon} w_{0}\right\| \lesssim \varepsilon\left\|H^{1}\right\|_{T, 2}+\sqrt{\varepsilon}\left\|w_{0}\right\| .
\end{aligned}
$$

By the same method, we get

$$
\begin{aligned}
\left\|w^{\mathrm{app}}\right\|_{T, \infty}=\left\|G^{\mathrm{app}} \mathcal{F}\right\|_{T, \infty} & \left.=\varepsilon^{-\frac{1}{2}} \| O p_{g} \overline{\bar{\kappa}}^{l}\left(\varepsilon \partial_{y}\right) \mathcal{M}_{\varepsilon} \mathcal{D}_{\varepsilon} \mathcal{F}\left(\tau^{\prime}, Z\right) d \tau^{\prime}\right) \|_{T / \varepsilon, \infty} \\
& \lesssim \varepsilon^{\frac{1}{2}}\left\|\mathcal{M}^{\varepsilon} H^{1}\right\|_{T / \varepsilon, 2}+\left\|M_{\varepsilon} w_{0}\right\| \lesssim \varepsilon^{\frac{1}{2}}\left\|H^{1}\right\|_{T, 2}+\left\|w_{0}\right\| .
\end{aligned}
$$

In a similar way, since

$$
\partial_{t} \mathcal{M}_{\varepsilon}^{-1}=\varepsilon^{-1} \mathcal{M}_{\varepsilon}^{-1} \partial_{\tau}, \quad \partial_{z} \mathcal{M}_{\varepsilon}^{-1}=\varepsilon^{-1} \mathcal{M}_{\varepsilon}^{-1} \partial_{Z}, \quad \partial_{Z} \mathcal{M}_{\varepsilon}=\varepsilon \mathcal{M}_{\varepsilon} \partial_{z}
$$

we find

$$
\begin{aligned}
\left\|\partial_{z}\left(G^{\mathrm{app}} \mathcal{F}\right)\right\|_{T, 2} & \lesssim\left\|H^{1}\right\|_{T, 2}+\varepsilon^{-\frac{1}{2}}\left\|w_{0}\right\|, \\
\left\|\partial_{t}\left(G^{\mathrm{app}} \mathcal{F}\right)\right\|_{T, 2} & \lesssim\left\|H^{1}\right\|_{T, 2}+\varepsilon^{-\frac{1}{2}}\left\|w_{0}\right\|+\varepsilon^{\frac{1}{2}}\left\|\nabla w_{0}\right\| \\
\left\|\partial_{z z}\left(G^{\mathrm{app}} \mathcal{F}\right)\right\|_{T, 2} & \lesssim \varepsilon^{-1}\left\|H^{1}\right\|_{T, 2}+\varepsilon^{-\frac{3}{2}}\left\|w_{0}\right\|+\varepsilon^{-\frac{1}{2}}\left\|\nabla w_{0}\right\|, \\
\left\|\partial_{z}\left(G^{\mathrm{app}} \mathcal{F}\right)\right\|_{T, \infty} & \lesssim \varepsilon^{-\frac{1}{2}}\left\|H^{1}\right\|_{T, 2}+\varepsilon^{-1}\left\|w_{0}\right\|+\left\|\nabla w_{0}\right\| .
\end{aligned}
$$

Consequently, we define the weighted norm $N_{T}^{\varepsilon}(u)$ by

$$
\begin{aligned}
& \quad N_{T}^{\varepsilon}(u)=\varepsilon^{-1}\|u\|_{T, 2}+\|\nabla u\|_{T, 2}+\left\|\partial_{t} u\right\|_{T, 2}+\varepsilon\left\|\nabla^{2} u\right\|_{T, 2}+\varepsilon^{-\frac{1}{2}}\|u\|_{T, \infty}+\varepsilon^{\frac{1}{2}}\|\nabla u\|_{T, \infty} . \\
& 4^{\text {e }} \text { SÉRIE - TOME } 41 \text { - } 2008 \text { - } \mathrm{N}^{\mathrm{o}} 6
\end{aligned}
$$


Note that the norm $N_{T}^{\varepsilon}(w)$ involves $\nabla w$ in our definition. Nevertheless, since we use only this norm for functions whose Fourier transform in $y$ is supported in $r \leq \varepsilon|\xi| \leq R$, the terms involving $\nabla$ in the norm actually give a useful, non redondant piece of information for $\partial_{z}$ only.

We also define the weighted norm $\|\mathcal{F}\|_{T, \varepsilon}$ as

$$
\|\mathcal{F}\|_{T, \varepsilon}=\left\|H^{1}\right\|_{T, 2}+\varepsilon^{-\frac{1}{2}}\left\|w_{0}\right\|+\varepsilon^{\frac{1}{2}}\left\|\nabla w_{0}\right\|
$$

we have actually proven that

$$
N_{T}^{\varepsilon}\left(G^{\text {app }} \mathcal{F}\right) \lesssim\|\mathcal{F}\|_{T, \varepsilon}
$$

Moreover, by using again Lemma 10 and Lemma 15, we get by the same method that

$$
N_{T}^{\varepsilon}\left(\mathrm{Op}_{D_{y}^{\alpha} g^{\text {app }}} \mathcal{F}\right) \lesssim C_{\alpha}\|\mathcal{F}\|_{T, \varepsilon}
$$

We can now check that $w^{\text {app }}$ is a suitable approximate solution. To have clear notations in the following computation, we use the notation

$$
\mathrm{Op}_{\mathcal{T}}=\mathcal{T}\left(q(0, y), \varepsilon D_{y}\right) .
$$

To check that $G^{\text {app }}$ is a good approximate solution, we write

$$
\begin{aligned}
\frac{1}{\varepsilon} \mathcal{M}_{\varepsilon}^{-1} \mathbb{P}_{+}\left(\varepsilon D_{y}\right) \bar{\kappa}^{l}( & \left.\varepsilon D_{y}\right) \operatorname{Op}_{\mathcal{T}} \mathcal{M}_{\varepsilon} \mathrm{Op}_{g^{\mathrm{app}}} \mathcal{F}+\mathcal{R}^{\varepsilon} \mathrm{Op}_{g^{\text {app }}} \mathcal{F} \\
& =\frac{1}{\varepsilon} \mathcal{M}_{\varepsilon}^{-1} \mathbb{P}_{+}\left(\varepsilon D_{y}\right) K^{l}\left(\varepsilon D_{y}\right) \mathrm{Op}_{\mathcal{T}} \mathcal{M}_{\varepsilon} \mathrm{Op}_{g^{\mathrm{app}}} \mathcal{F}+\mathcal{R}^{\varepsilon} \mathrm{Op}_{g^{\mathrm{app}}} \mathcal{F}+\mathcal{C}^{r}
\end{aligned}
$$

where $K^{l}(\xi)$ is a smooth compactly supported function with a support slightly bigger than the one of $\overline{\bar{\kappa}}$ and such that

$$
K^{l} \overline{\bar{\kappa}}=\overline{\bar{\kappa}}
$$

The commutator $\mathcal{C}^{r}$ is defined by

$$
\mathcal{C}^{r}=\frac{1}{\varepsilon} \mathcal{M}_{\varepsilon}^{-1} \mathbb{P}_{+}\left(\varepsilon D_{y}\right) K^{l}\left[\bar{\kappa}, \mathrm{Op}_{\mathcal{T}}\right] \mathrm{Op}_{g^{\text {app }}} \mathcal{F}
$$

and hence is very similar to $\mathcal{C}$. In particular, thanks to (85), we have

$$
\left\|\mathcal{C}^{r}\right\|_{T, 2} \lesssim \varepsilon\|\mathcal{F}\|_{T, \varepsilon}
$$

Next, we write

$$
\begin{aligned}
\frac{1}{\varepsilon} \mathcal{M}_{\varepsilon}^{-1} \mathbb{P}_{+} & \left(\varepsilon D_{y}\right) K^{l}\left(\varepsilon D_{y}\right) \mathrm{Op}_{\mathcal{T}} \mathcal{M}_{\varepsilon} \mathrm{Op}_{g^{\mathrm{app}}} \mathcal{F}+\mathcal{R}^{\varepsilon} \mathrm{Op}_{g^{\mathrm{app}}} \mathcal{F}+\mathcal{C}^{r} \\
= & \frac{1}{\varepsilon} \mathcal{M}_{\varepsilon}^{-1} \mathrm{Op}_{\mathbb{P}_{+} K^{l}} \mathrm{Op}_{\mathcal{T} \mathcal{M}_{\varepsilon} g^{\mathrm{app}}} \mathcal{F}+\mathcal{R}^{\varepsilon} \mathrm{Op}_{g^{\mathrm{app}}} \mathcal{F}+\mathcal{C}^{r}+\mathcal{R}^{1} \mathcal{F} \\
& =\frac{1}{\varepsilon} \mathcal{M}_{\varepsilon}^{-1} \mathrm{Op}_{\mathbb{P}_{+} K^{l} \mathcal{T} g} \mathcal{M}_{\varepsilon} \mathcal{D}_{\varepsilon} \mathcal{F}+\mathcal{R}^{\varepsilon} \mathrm{Op}_{g^{\mathrm{app}}} \mathcal{F}+\mathcal{C}^{r}+\mathcal{R}^{1} \mathcal{F}+\mathcal{R}^{2} \mathcal{F}
\end{aligned}
$$

Since by definition, the symbol $g$, is chosen such that

$$
\mathbb{P}_{+}(\xi) K^{l}(\xi) \mathcal{T}(q(0, y), \xi) g(y, \xi)=K^{l}(\xi) I d,
$$


we get thanks to (82) and (87) that

$$
\begin{aligned}
& \frac{1}{\varepsilon} \mathcal{M}_{\varepsilon}^{-1} \mathbb{P}_{+}\left(\varepsilon D_{y}\right) \bar{\kappa}\left(\varepsilon D_{y}\right) \mathrm{Op}_{\mathcal{T}} \mathcal{M}_{\varepsilon} \mathrm{Op}_{g^{\mathrm{app}}} \mathcal{F}+\mathcal{R}^{\varepsilon} \mathrm{Op}_{g^{\mathrm{app}}} \mathcal{F} \\
& \quad=K^{l}\left(\varepsilon D_{y}\right) H^{1}+\mathcal{R}^{\varepsilon} \mathrm{Op}_{g^{\mathrm{app}}} \mathcal{F}+\mathcal{C}^{r}+\mathcal{R}^{1} \mathcal{F}+\mathcal{R}^{2} \mathcal{F}=H^{1}+\mathcal{C}^{r}+\mathcal{R}^{1} \mathcal{F}+\mathcal{R}^{2} \mathcal{F}
\end{aligned}
$$

The remainder $\mathcal{R}^{1} \mathcal{F}$ is defined by

$$
\mathcal{R}^{1} \mathcal{F}=\frac{1}{\varepsilon} \mathcal{M}_{\varepsilon}^{-1} \mathbb{P}_{+}\left(\varepsilon D_{y}\right) \bar{\kappa}\left(\varepsilon D_{y}\right) \mathrm{Op}_{r^{1}} \mathcal{F}
$$

with the symbol $r^{1}$ given by

$$
r^{1}(y, \xi)=\left(V(t / \varepsilon, q(t, y), Z) \cdot \varepsilon D_{y}+\varepsilon^{2} D_{y}^{2}\right) g^{\mathrm{app}}(y, \xi) .
$$

Consequently, thanks to (86), we have

$$
\left\|\mathcal{R}^{1} \mathcal{F}\right\|_{T, 2} \lesssim \varepsilon\|\mathcal{F}\|_{T, \varepsilon}
$$

In a similar way, thanks to Lemma 16 and (86), we have for $m>7$

$$
\left\|\mathcal{R}^{2} \mathcal{F}\right\|_{T, 2} \lesssim \varepsilon \sum_{|\alpha| \leq 2} N^{\varepsilon}\left(\mathrm{Op}_{D_{y}^{\alpha} g^{\mathrm{app}}} \mathcal{F}\right) \lesssim \varepsilon\|\mathcal{F}\|_{T, \varepsilon}
$$

Finally, thanks to (75), we also have

$$
\left\|\mathcal{R}^{\varepsilon} \mathrm{Op}_{g^{\text {app }}} \mathcal{F}\right\|_{T, 2} \lesssim(\delta+T)\|\mathcal{F}\|_{T, \varepsilon}
$$

To use the result of Lemma 9, we set

$$
R^{\mathrm{app}} \mathcal{F}=\mathcal{R}^{\varepsilon} G^{\mathrm{app}}\left(y, \varepsilon D_{y}\right) H^{1}+\mathcal{C}^{r}+\mathcal{R}^{1} \mathcal{F}+\mathcal{R}^{2} \mathcal{F}
$$

and we see thanks to (88), (89), (90), (91) that

$$
\left\|R^{\text {app }} \mathcal{F}\right\|_{T, 2} \lesssim(\delta+T+\varepsilon)\|\mathcal{F}\|_{T, \varepsilon} .
$$

Consequently, thanks to Lemma 9 , for $\varepsilon$ and $\delta$ sufficiently small, there exist $T^{0}$ and $C^{0}>0$ such that

$$
N_{T^{0}}^{\varepsilon}(w) \leq C^{0}\|\mathcal{F}\|_{T^{0}, \varepsilon} .
$$

To get an estimate on a longer interval of time, we can reiterate the process as long as $q(t, y) \in \mathcal{K}$. Indeed, since $q\left(T^{0}, y\right) \in \mathcal{K}$, then we can use the same method as previously for $T \leq T^{0}$; we rewrite the analogous of (74) but we replace $\mathcal{T}\left(w^{\text {int, } 0}(0, y), \varepsilon D_{y}\right)$ by $\mathcal{T}\left(w^{\text {int }, 0}\left(T^{0}, y\right), \varepsilon D_{y}\right)$. The same argument as previously allows to get an estimate on $\left[T^{0}, 2 T^{0}\right]$. The iteration of the argument finally allows to get for some $C>0, \gamma_{0}>0$ independent of $T$, that

$$
N_{T}^{\varepsilon}(w) \leq C e^{\gamma_{0} T}\|\mathcal{F}\|_{T, \varepsilon}
$$

for every $T>0$ such that $w^{\text {int }}(t, y, 0) \in \mathcal{K}$, for $t \in[0, T]$.

$4^{\mathrm{e}}$ SÉRIE - TOME $41-2008-\mathrm{N}^{\mathrm{o}} 6$ 
Finally, we can multiply (93) by $\varepsilon^{\frac{1}{2}}$, use that $w=\chi^{b} v^{l}-d$ and the estimates (43), (46), (47), (66), (67), (59), (60) to get in particular that

$$
\begin{aligned}
\left\|\chi^{b} v^{l}(T)\right\|^{2}+ & \int_{0}^{T}\left(\varepsilon^{-1}\left\|\chi^{b} v^{l}\right\|^{2}+\varepsilon\left\|\nabla\left(\chi^{b} v^{l}\right)\right\|^{2}\right) \\
\lesssim & e^{\gamma_{0} T}\left\|v_{0}^{l}\right\|_{1, \varepsilon}^{2}+\varepsilon e^{\gamma_{0} T} \int_{0}^{T}\|F\|^{2} \\
& +\varepsilon e^{\gamma_{0} T} \int_{0}^{T}\left(\|v\|^{2}+\varepsilon^{2}\|\nabla v\|^{2}+\varepsilon^{4}\left\|\Delta v^{l}\right\|^{2}+\varepsilon^{2}\left\|\partial_{t} v^{l}\right\|^{2}\right) .
\end{aligned}
$$

This ends the proof of (52).

We can now end the proof of Proposition 8. At this point, we shall restrict $\varepsilon$ and $T$ such that $\varepsilon e^{\gamma_{0} T} \lesssim 1$; this will allow to absorb the terms in the right hand side still depending on $v^{l}$ which involve higher order derivatives by another estimates. At first, we can use the decompositions (40) and (52), (49) (we recall that we get an estimate near the upper boundary $z=1$ completely analogous to (52)), to get

$$
\begin{aligned}
& \left\|v^{l}(T)\right\|^{2}+\int_{0}^{T}\left(\varepsilon^{-1}\left\|v^{l}\right\|^{2}+\varepsilon\left\|\nabla v^{l}\right\|^{2}\right) \\
& \lesssim e^{\gamma_{0} T}\left\|v^{l}(0)\right\|_{1, \varepsilon}^{2}+\varepsilon e^{\gamma_{0} T} \int_{0}^{T}\|F\|^{2} \\
& \quad+\left(1+\varepsilon e^{\gamma_{0} T}\right) \int_{0}^{T}\left(\|v\|^{2}+\varepsilon^{2}\|\nabla v\|^{2}+\varepsilon^{4}\left\|\Delta v^{l}\right\|^{2}+\varepsilon^{2}\left\|\partial_{t} v^{l}\right\|^{2}\right) .
\end{aligned}
$$

To conclude, we use Lemma 6 to estimate higher order derivatives. We get

$$
\begin{aligned}
& \varepsilon^{2}\left\|\nabla v^{l}(T)\right\|^{2}+\int_{0}^{T}\left(\varepsilon\left\|\partial_{t} v^{l}\right\|^{2}+\varepsilon^{3}\left\|\nabla^{2} v\right\|^{2}\right) \\
& \lesssim \varepsilon^{2}\left\|\nabla v_{0}\right\|^{2}+\int_{0}^{T}\left(\varepsilon^{-1}\left\|v^{l}\right\|^{2}+\varepsilon\left\|\nabla v^{l}\right\|^{2}+\varepsilon\|v\|^{2}+\varepsilon^{2}\|\nabla v\|^{2}+\varepsilon\|F\|^{2}\right) .
\end{aligned}
$$

Finally we can add (97) and (95) times a sufficiently large constant to get

$$
\begin{aligned}
\left\|v^{l}(T)\right\|^{2}+ & \varepsilon^{2}\left\|\nabla v^{l}\right\|^{2}+\int_{0}^{T}\left(\varepsilon^{-1}\left\|v^{l}\right\|^{2}+\varepsilon\left\|\nabla v^{l}\right\|^{2}+\varepsilon\left\|\partial_{t} v^{l}\right\|^{2}+\varepsilon^{3}\left\|\nabla^{2} v^{l}\right\|^{2}\right) \\
\lesssim & e^{\gamma_{0} T}\left\|v_{0}\right\|_{1, \varepsilon}^{2}+\varepsilon e^{\gamma_{0} T} \int_{0}^{T}\|F\|^{2} \\
& +\left(1+\varepsilon e^{\gamma_{0} T}\right) \int_{0}^{T}\left(\|v\|^{2}+\varepsilon^{2}\|\nabla v\|^{2}+\varepsilon^{4}\left\|\Delta v^{l}\right\|^{2}+\varepsilon^{2}\left\|\partial_{t} v^{l}\right\|^{2}\right)
\end{aligned}
$$

and hence, for $\varepsilon$ sufficiently small, and $\varepsilon e^{\gamma_{0} T} \leq 1$, we finally get the result of Proposition 8 . 


\subsection{End of the proof of Theorem 4}

To get (30), we collect the estimates of Propositions 5, 7, 8. We get for $\varepsilon, T$ such that $\varepsilon e^{\gamma_{0} T} \leq 1$ :

$$
\begin{aligned}
& \|v(T)\|^{2}+\left\|\nabla v^{H F}(T)\right\|_{1, \varepsilon}^{2}+\int_{0}^{T}\left(\varepsilon\|\nabla v\|^{2}+\varepsilon^{-1}\left\|v^{H F}\right\|^{2}\right) \\
& \lesssim e^{\gamma_{0} T}\left(\left\|v_{0}\right\|^{2}+\left\|v_{0}^{H F}\right\|_{1, \varepsilon}^{2}\right)+\int_{0}^{T}\left(\varepsilon e^{\gamma_{0} T}\|F\|^{2}+\|v\|^{2}+\varepsilon^{2}\|\nabla v\|^{2}+\left|\left(\kappa^{s} F, \kappa^{s} v\right)\right|\right) .
\end{aligned}
$$

For $\varepsilon$ sufficiently small, this gives

$$
\begin{aligned}
& \|v(T)\|^{2}+\left\|\nabla v^{H F}\right\|_{1, \varepsilon}^{2}+\int_{0}^{T}\left(\varepsilon\|\nabla v\|^{2}+\varepsilon^{-1}\left\|v^{H F}\right\|^{2}\right) \\
& \lesssim e^{\gamma_{0} T}\left(\left\|v_{0}\right\|^{2}+\left\|v_{0}^{H F}\right\|_{1, \varepsilon}^{2}\right)+\int_{0}^{T}\left(\varepsilon e^{\gamma_{0} T}\|F\|^{2}+\|v\|^{2}+\left|\left(\kappa^{s} F, \kappa^{s} v\right)\right|\right)
\end{aligned}
$$

and hence Theorem 4 follows by using the Gronwall inequality.

\subsection{Proof of Lemma 9}

We represent the exact solution $w$ of (69) as

$$
w=\sum_{k \geq 0} w^{k}
$$

where

$$
w^{0}=G^{\mathrm{app}}\left(F, w_{0}\right), \quad R^{0}=R^{\mathrm{app}}\left(F, w^{0}\right)
$$

and for $k \geq 1$ we define recursively $w^{k}$ and $R^{k}$ as

$$
w^{k}=-G^{\mathrm{app}}\left(R^{k-1}, 0\right), \quad R^{k}=R^{\mathrm{app}}\left(R^{k-1}, 0\right) .
$$

Thanks to (70), (71), we easily get by induction that

$$
N_{T}^{\varepsilon}\left(w^{k}\right) \leq C_{T, \varepsilon}\left(C_{T, \varepsilon}^{1}\right)^{k}\left\|\left(F, w_{0}\right)\right\|_{T, \varepsilon}, \quad\left\|R^{k}\right\|_{T, \varepsilon} \leq\left(C_{T, \varepsilon}^{1}\right)^{k+1}\left\|\left(F, w_{0}\right)\right\|_{T, \varepsilon}
$$

and hence, thanks to (72), we get that

$$
\left.N_{T}^{\varepsilon}(w) \leq C_{T, \varepsilon}\left(\sum_{k \geq 0}\left(C_{T, \varepsilon}^{1}\right)^{k}\right)\right)\left\|\left(F, w_{0}\right)\right\|_{T, \varepsilon} \leq C_{T, \varepsilon} C\left\|\left(F, w_{0}\right)\right\|_{T, \varepsilon}
$$

\subsection{Proof of Lemma 10}

We start with the proof for $m=0$. In this section, $\lesssim$ means $\leq C$ where $C$ is independent of $\Theta$.

We can write the solution of (78) under the form

$$
G(q, \xi) \mathcal{F}(\tau)=\mathbb{S}_{+}(\tau, 0, q, \xi) \overline{\bar{\kappa}}^{l}(\xi) w_{0}+\int_{0}^{\tau} \mathbb{S}_{+}\left(\tau, \tau^{\prime}, q, \xi\right) \overline{\bar{\kappa}}^{l}(\xi) F\left(\tau^{\prime}\right) d \tau^{\prime}
$$

and hence, thanks to $(\mathrm{H})$, we get that

$$
|G(q, \xi) \mathcal{F}(\tau)| \lesssim e^{-\alpha \tau}\left|w_{0}\right|+\int_{0}^{\tau} e^{-\alpha\left(\tau-\tau^{\prime}\right)}\left|F\left(\tau^{\prime}\right)\right| d \tau^{\prime} .
$$

This yields by standard results on convolutions that

$$
\|G(q, \xi) \mathcal{F}(\tau)\|_{\Theta, \infty} \lesssim\left|w_{0}\right|+\|F\|_{\Theta, 2}
$$

$4^{\mathrm{e}}$ SÉRIE - TOME $41-2008-\mathrm{N}^{\mathrm{o}} 6$ 
and that

$$
\|G(q, \xi) \mathcal{F}(\tau)\|_{\Theta, 2} \lesssim\left|w_{0}\right|+\|F\|_{\Theta, 2} .
$$

Next, we can reintroduce the pressure and rewrite the equation (78) as

$$
\begin{aligned}
& \partial_{\tau} u+V(\tau, Z, q) \cdot\left(\begin{array}{c}
i \xi \\
\partial_{Z}
\end{array}\right) u+u \cdot\left(\begin{array}{c}
i \xi \\
\partial_{Z}
\end{array}\right) V(\tau, Z, q)+\left(\begin{array}{c}
i \xi \\
\partial_{Z}
\end{array}\right) p \\
& \quad+e \times u+|\xi|^{2} u-\partial_{Z Z} u=0
\end{aligned}
$$

with the divergence free condition

$$
i \xi \cdot u_{h}+\partial_{Z} u_{3}=0
$$

Consequently, the standard energy estimate gives

$$
|u(\tau)|^{2}+\int_{0}^{\tau}\left|\partial_{Z} u\right|^{2} \lesssim\left|u_{0}\right|^{2}+\int_{0}^{\tau}|F|^{2}+\int_{0}^{\tau}|u|^{2}
$$

and since the right-hand side is already estimated thanks to (99), we also get

$$
\left\|\partial_{Z} G(q, \xi) \mathcal{F}(\tau)\right\|_{\Theta, 2} \lesssim\left|w_{0}\right|+\|F\|_{\Theta, 2}
$$

To estimate higher order derivatives, we use again Lemma 6 (with $\varepsilon=1$ ); we get

$$
\left\|\partial_{Z} u\right\|_{\Theta, \infty}^{2}+\left\|\partial_{\tau} u\right\|_{\Theta, 2}^{2}+\left\|\partial_{Z Z} u\right\|_{\Theta, 2}^{2} \lesssim\left|\partial_{Z} u_{0}\right|^{2}+\|F\|_{\Theta, 2}^{2}+\|u\|_{\Theta, 2}^{2}
$$

and since the right-hand side is again already bounded thanks to (99), we get that

$$
\left\|\partial_{Z} G(q, \xi) \mathcal{F}\right\|_{\Theta, \infty}+\left\|\partial_{\tau} G(q, \xi) \mathcal{F}\right\|_{\Theta, 2}+\left\|\partial_{Z Z} G(q, \xi) \mathcal{F}\right\|_{\Theta, 2} \lesssim\left|w_{0}\right|+\|F\|_{\Theta, 2} .
$$

This ends the proof of (83), (84) for $m=0$.

The general case follows by induction, we shall just explain how to handle the case $m=1$. The regularity of the solution of (78) with respect to $q$ follows from standard regularity results for solutions of parabolic equations whose coefficients smoothly depend on a parameter [10]. Taking the differential of (78) with respect to $q$ in the direction $h$, we find that

$$
\left(\partial_{\tau}-\mathbb{P}_{+}(\xi) \mathbb{L}_{+}(\tau, q, \xi)\right) D_{q} u \cdot h=R^{1}
$$

with

$$
R^{1}=-\left(\left(D_{q} V \cdot h\right) \cdot\left(\begin{array}{c}
i \xi \\
\partial_{Z}
\end{array}\right) u+u \cdot\left(\begin{array}{c}
i \xi \\
\partial_{Z}
\end{array}\right) D_{q} V \cdot h\right)
$$

and $D_{q} u \cdot h_{/ t=0}=0$. Consequently, we have

$$
D_{q} u \cdot h=G(q, \xi)\left(R^{1}, 0\right) .
$$

By using (83), (84) for $m=0$, we get

$$
\begin{aligned}
& \left|D_{q} u \cdot h\right|_{\Theta, \infty}+\left|D_{q} u \cdot h\right|_{\Theta, 2}+\left|\partial_{Z} D_{q} u \cdot h\right|_{\Theta, 2} \\
& +\left|\partial_{Z} D_{q} u \cdot h\right|_{\Theta, \infty}+\left|\partial_{\tau} D_{q} u \cdot h\right|_{\Theta, 2}+\left|\partial_{Z Z} D_{q} u \cdot h\right|_{\Theta, 2} \\
& \lesssim\left|R^{1}\right|_{\Theta, 2} \lesssim|h|\left(|u|_{\Theta, 2}+\left|\partial_{Z} u\right|_{\Theta, 2}\right) .
\end{aligned}
$$

Consequently, we can use again (83), (84) for $m=0$ to get the result for $m=1$. 


\subsection{Estimates of the gradient}

The aim of this section is to estimate $\|\nabla v(T)\|^{2}$, we first give a crude estimate:

THEOREM 11. - Under the assumptions of Theorem 4, we have

$$
\begin{aligned}
& \varepsilon^{3}\|\nabla v(T)\|^{2}+\int_{0}^{T}\left(\varepsilon^{2}\left\|\partial_{t} v\right\|^{2}+\varepsilon^{4}\left\|\nabla^{2} v\right\|^{2}+\varepsilon^{2}\|\nabla p\|^{2}\right) \\
& \lesssim \varepsilon^{3}\left\|\nabla v_{0}\right\|^{2}+e^{\gamma T}\left(\left\|v_{0}\right\|^{2}+\left\|v^{H F}\right\|_{1, \varepsilon}^{2}+\int_{0}^{T}\left|\left(\kappa^{s} F, \kappa^{s} v\right)\right|+\varepsilon \int_{0}^{T}\|F\|^{2}\right)
\end{aligned}
$$

Note that this estimate is relatively crude since we only have a control of $\varepsilon^{3}\|\nabla v(T)\|^{2}$ whereas, because of the size of the boundary layers, we would expect a control of $\varepsilon^{2}\|\nabla v(T)\|^{2}$ as we had for the large frequency part of the solution. Nevertheless, this estimate will be useful in Section 4. The reason is that in the proof we do not use in an optimal way the structure of the singular term $\varepsilon^{-1} e \times v$.

\section{Proof of Theorem 11}

To get (102), it suffices to use Lemma 6, then multiply the estimate (35) by $\varepsilon$ and finally use (30).

To get better estimates of some components of $\nabla v$, we shall rewrite the equation (28) under an equivalent form which is classically used in fluid mechanics. We define $\eta=\partial_{1} v_{2}-\partial_{2} v_{1}$ and $w=v_{3}$. Note that $\eta$ is the third component of the curl of $v$. Taking the curl of (28) and using that $v$ is divergence-free, we easily get that the equation for $\eta$ is given by

$$
\begin{aligned}
& \partial_{t} \eta-\frac{\partial_{z} w}{\varepsilon}-\varepsilon \Delta \eta \\
& =\partial_{1}\left(u^{\mathrm{app}} \cdot \nabla v_{2}+v \cdot \nabla u_{2}^{\mathrm{app}}+F_{2}\right)-\partial_{2}\left(u^{\mathrm{app}} \cdot \nabla v_{1}+v \cdot \nabla u_{1}^{\mathrm{app}}+F_{1}\right) .
\end{aligned}
$$

For the equation on $w$, we first derive the equation for the pressure. We take the divergence of (28) to get

$$
\Delta p=\frac{\eta}{\varepsilon}+\nabla \cdot\left(F-u^{\mathrm{app}} \cdot \nabla v-v \cdot \nabla u^{\mathrm{app}}\right)
$$

and next, we take the Laplacian in the third component of (28) and we use (104) to get

$$
\partial_{t} \Delta w+\frac{\partial_{z} \eta}{\varepsilon}-\varepsilon \Delta^{2} w=\left(\Delta \circ \pi_{3}-\partial_{z} \nabla \cdot\right)\left(-u^{\mathrm{app}} \cdot \nabla v-v \cdot \nabla u^{\mathrm{app}}+F\right)
$$

where $\pi_{3}$ stands for the projection on the third component i.e. $\pi_{3}(v)=v_{3}$. Next, thanks to (2) and the fact that $\nabla \cdot w=0$, we notice that the boundary condition for (103), (105) is given by

$$
\eta_{/ \partial \Omega}=w_{/ \partial \Omega}=\partial_{z} w_{/ \partial \Omega}=0 .
$$

For the system (103), (105), we can prove: 
THEOREM 12. - Under the same assumptions as in Theorem 4, we have

$$
\varepsilon^{2}\left(\|\eta(T)\|^{2}+\|\nabla w(T)\|^{2}\right) \lesssim e^{\gamma T}\left(\left\|v_{0}\right\|_{1, \varepsilon}^{2}+\int_{0}^{T} \mid\left(\kappa^{s} F, \kappa^{s} v \mid+\varepsilon \int_{0}^{T}\|F\|^{2}\right)\right.
$$

and also

$$
\varepsilon^{2}\left(\left\|\nabla_{h} v_{h}(T)\right\|^{2}+\|\nabla w(T)\|^{2}\right) \lesssim e^{\gamma T}\left(\left\|v_{0}\right\|_{1, \varepsilon}^{2}+\int_{0}^{T}\left|\left(\kappa^{s} F, \kappa^{s} v\right)\right|+\varepsilon \int_{0}^{T}\|F\|^{2}\right)
$$

for some $\gamma>0$.

Proof. - To estimate the solution of (103), (105), we multiply (103) by $\eta$ and (105) by $-w$ and we add the two equations. We use (106) to get thanks to integration by parts that

$$
\begin{aligned}
& \frac{d}{d t} \frac{1}{2}\left(\|\eta\|^{2}+\|\nabla w\|^{2}\right)+\varepsilon\left(\|\nabla \eta\|^{2}+\|\Delta w\|^{2}\right)-\varepsilon^{-1}\left(\left(\partial_{z} w, \eta\right)+\left(\partial_{z} \eta, w\right)\right) \\
& \lesssim\left(\|\nabla v\|+\varepsilon^{-1}\left\|v \partial_{Z} u^{b}\right\|+\|F\|\right)(\|\nabla \eta\|+\|\Delta w\|) .
\end{aligned}
$$

The crucial fact in the above identity is that

$$
\left(\partial_{z} w, \eta\right)+\left(\partial_{z} \eta, w\right)=0
$$

so that the singular term vanishes. Consequently, we can use the Young inequality, the estimate (38) and multiply by $\varepsilon^{2}$ to get

$$
\begin{aligned}
& \varepsilon^{2}\left(\|\eta(T)\|^{2}+\|\nabla w(T)\|^{2}\right)+\varepsilon^{3} \int_{0}^{T}\left(\|\nabla \eta\|^{2}+\|\Delta w\|^{2}\right) \\
& \lesssim \varepsilon^{2}\left(\left\|\eta_{0}\right\|^{2}+\left\|\nabla w_{0}\right\|^{2}\right)+\int_{0}^{T}\left(\varepsilon\|\nabla v\|^{2}+\|v\|^{2}+\varepsilon\|F\|^{2}\right) .
\end{aligned}
$$

Since the right hand side of the above estimate was already estimated in (30), we get (107).

To get (108), we use that $\nabla \cdot v=0$, to get

$$
\Delta_{h} v_{2}=\partial_{1} \eta-\partial_{2} \partial_{z} w, \quad \Delta_{h} v_{1}=-\partial_{2} \eta-\partial_{1} \partial_{z} w, \quad \Delta_{h}=\partial_{1}^{2}+\partial_{2}^{2} .
$$

This immediately yields that

$$
\left\|\nabla_{h} v_{i}\right\| \lesssim\|\eta\|+\left\|\partial_{z} w\right\|, \quad i=1,2
$$

and hence (108) follows from (107).

\section{Higher order conormal derivatives}

The estimates of Theorem 4 are sufficient to get a nonlinear stability result when it is possible to construct a very accurate approximate solution. Indeed, we can very easily estimate weighted derivatives under the form $\varepsilon^{|\alpha|} \partial_{y}^{\alpha}$. It suffices to apply the operator $\varepsilon^{|\alpha|} \partial_{y}^{\alpha}$ to (28), to rewrite the obtained equation as

$$
\mathcal{T}^{\varepsilon}\left(\varepsilon^{|\alpha|} \partial_{y}^{\alpha} v, \varepsilon^{|\alpha|} \partial_{y}^{\alpha} p\right)=\varepsilon^{|\alpha|} \partial_{y}^{\alpha} F+\mathcal{C}
$$

where $\mathcal{C}$ is a well-controlled commutator and then to apply Theorem 4 . The drawback of this approach is that this yields by Sobolev embedding a bad control of the $L^{\infty}$ norm which is needed to prove the nonlinear stability by a fixed point argument. Since here we have been 
only able to construct an approximate solution with $\left\|R^{\varepsilon}\right\| \lesssim \varepsilon$, we cannot use this rough approach to conclude.

In order to conclude, we would want to prove as it was done in [26], [28] that we can estimate tangential derivatives, i.e. $\partial_{y} v$ where $v$ is still the solution of (28) without loss. This means that we want to estimate $\left\|\partial_{y}^{\alpha} v\right\|$ and not $\varepsilon^{|\alpha|}\left\|\partial_{y}^{\alpha} v\right\|$. Here a new difficulty appears which was not present in the well-prepared case. Indeed, when we apply $\partial_{y}$ to (28), we have in particular to handle the commutator

$$
\left[\partial_{y},\left(u^{\mathrm{int}}+u^{b}\right) \cdot \nabla\right] v=\partial_{y} u^{\mathrm{int}} \cdot \nabla v+\partial_{y} u^{b} \cdot \nabla v .
$$

The second term has the same property as in the well-prepared case since $u_{3}^{b}=0$, we get that

$$
\left\|\partial_{y} u^{b} \cdot \nabla v\right\|^{2} \lesssim\left\|\nabla_{h} v\right\|^{2}
$$

and hence this term can be handled by a Gronwall type argument since it involves only first order tangential derivatives. The main difficulty comes from the term $\partial_{y} u^{\text {int }} \cdot \nabla v$. In the wellprepared case, we have $u_{3}^{\text {int }}=0$ and hence the same argument as above is valid. Nevertheless, here we do not have $u_{3}^{\text {int }}=0$, we only have $u_{3 / \partial \Omega}^{\text {int }}=0$. Consequently, we have the estimate

$$
\left\|\partial_{y} u_{3}^{\mathrm{int}} \partial_{z} v\right\| \lesssim\left\|\varphi \partial_{z} v\right\|
$$

where $\varphi(z)$ is a smooth bounded function which behaves as $z$ and $1-z$ in the vicinity of $z=0$ and $z=1$ respectively. The usual method in the case of initial boundary value problems for viscous conservation laws (see [24], [19], for example) is to work in conormal spaces and to consider simultaneously the derivatives in the directions tangent to the boundary and the additional vector field $\varphi \partial_{z}$. Note that it is legitimate to apply this last vector field $\varphi \partial_{z}$ to the equation since $\left(\varphi \partial_{z} v\right)_{/ \partial \Omega}=0$. Nevertheless, in the case of our singularly perturbed incompressible Navier-Stokes equation, it does not seem easy to use readily this method. Indeed $\varphi \partial_{z} v$ does not verify the incompressibility condition and moreover $\nabla \cdot\left(\varphi \partial_{z} v\right)$ is not small. To overcome this difficulty, we shall use that $u_{3}^{\text {int }}$ is highly oscillating in time. We recall that $u_{3}^{\text {int }}$ is defined by

$$
u_{3}^{\mathrm{int}}=\sum_{\mathbf{k}} b(t, \mathbf{k}) e^{i \lambda(\mathbf{k}) \tau} e^{i k \cdot y} 2 i \sin \left(k_{3} z\right)=\sum_{\mathbf{k}, k_{3} \neq 0} b(t, \mathbf{k}) e^{i \lambda(\mathbf{k}) \tau} e^{i k \cdot y} 2 i \sin \left(k_{3} z\right) .
$$

Thanks to this definition, we introduce

$$
W_{j}^{\varepsilon}(t, x)=\sum_{\mathbf{k}, k_{3} \neq 0} k_{j} \frac{b(t, \mathbf{k})}{\lambda(\mathbf{k})} e^{i \lambda(\mathbf{k}) t / \varepsilon} e^{i k \cdot y} 2 i \sin \left(k_{3} z\right) .
$$

Note that we have

$$
\partial_{t} W_{j}^{\varepsilon}=\varepsilon^{-1} \partial_{j} u_{3}^{\mathrm{int}}+\mathcal{O}(1)
$$

and that

$$
W_{j / \partial \Omega}^{\varepsilon}=0
$$

Next, we introduce the vector fields

$$
Z_{j}=\partial_{j}+\varepsilon W_{j}^{\varepsilon}(t, x) \partial_{z}, j=1,2, \quad Z_{3}=\varepsilon \Gamma \varphi(z) \partial_{z}
$$

where $\Gamma \geq 1$ will be chosen sufficiently large. Since $\left|W_{3}^{\varepsilon}\right| \lesssim \varphi$ thanks to (110), we have

$$
\left|\partial_{j} v\right| \lesssim\left|Z_{j} v\right|+\left|Z_{3} v\right|
$$

$4^{\mathrm{e}}$ SÉRIE - TOME $41-2008-\mathrm{N}^{\circ} 6$ 
and hence we can recover good estimates on $\partial_{y} v$ from estimates on $Z v$. The main property of these vector fields as we shall see below is that they have good commutation property with respect to $\mathcal{T}$. Consequently, let us define the weighted norms

$$
\begin{aligned}
\|v\|_{m}^{2} & =\sum_{|\alpha| \leq m}\left\|Z_{3}^{\alpha_{3}} Z_{2}^{\alpha_{2}} Z_{1}^{\alpha_{1}} v\right\|^{2}, \\
Y_{m}(v) & =\|v\|_{m}^{2}+\varepsilon^{2}\left\|\nabla_{h} v_{h}\right\|_{m}^{2}+\varepsilon^{2}\left\|\nabla v_{3}\right\|_{m}^{2}+\varepsilon^{2}\left\|\nabla v^{H F}\right\|^{2}+\varepsilon^{3}\|\nabla v\|^{2}, \\
Y_{T, m}(v) & =\sup _{[0, T]} Y_{m}(v(T)), \\
D_{T, m}(v, p) & =\int_{0}^{T}\left(\varepsilon^{-1}\left\|v^{H F}\right\|_{m}^{2}+\varepsilon\|\nabla v\|_{m}^{2}+\varepsilon^{2}\left\|\partial_{t} v\right\|_{m}^{2}+\varepsilon^{2}\|\nabla p\|_{m}^{2}+\varepsilon^{4}\left\|\nabla^{2} v\right\|_{m}^{2}\right) .
\end{aligned}
$$

Note that the norm $\|\cdot\|_{m}$ that we have just defined is equivalent to the norm $\|\cdot\|_{m}$ defined in (18) because of (111), this is why we have abusively used the same notation. In order to deal with the source term in an optimal way, we also use the notation

$$
(u, v)_{m}=\sum_{|\alpha| \leq m}\left|\left(Z_{3}^{\alpha_{3}} Z_{2}^{\alpha_{2}} Z_{1}^{\alpha_{1}} u, Z_{3}^{\alpha_{3}} Z_{2}^{\alpha_{2}} Z_{1}^{\alpha_{1}} v\right)\right| .
$$

Our main result is:

THEOREM 13. - Under the same assumptions as in Theorem 4, we have for every $m$

$$
Y_{T, m}(v)+D_{T, m}(v, p) \lesssim e^{\gamma T}\left(Y_{m}\left(v_{0}\right)+\int_{0}^{T}\left(\kappa^{s} F, \kappa^{s} v\right)_{m}+\varepsilon \int_{0}^{T}\|F\|_{m}^{2}\right) .
$$

\section{Proof of Theorem 13}

We shall prove (112) by induction on $m$. In the proof, the harmless numbers contained in $\lesssim$ are also independent of $\Gamma \geq 1$.

Note that for $m=0$, the estimate (112) follows by collecting (102), (30), (108). To present the main idea without too much technicalities, we first give the proof of (112) for $m=1$. At first, let us study what happens to (28) when we apply the vector field $Z_{i}$ for $i=1,2$. The case where we apply $Z_{3}$ is easier because of the $\varepsilon$ weight in the vector field, and will not be detailed. Moreover, most of the terms which appear in the computation are similar to the ones which appear when we apply $Z_{i}$ to the equation since $\varepsilon W_{i}^{\varepsilon} \partial_{z}$ behaves in the same way as $Z_{3}$.

For $i=1,2$, we get

$$
\mathcal{T}^{\varepsilon}\left(Z_{i} v, Z_{i} p\right)=Z_{i} F-\mathcal{C}^{Z}
$$

where

$$
\begin{gathered}
\mathcal{C}^{Z}=\mathcal{C}_{1}^{Z}+\mathcal{C}_{2}^{Z}, \\
\mathcal{C}_{1}^{Z}=Z_{i} u^{\mathrm{app}} \cdot \nabla v-\varepsilon \partial_{t} W_{i}^{\varepsilon} \partial_{z} v \\
\mathcal{C}_{2}^{Z}=\varepsilon u^{\mathrm{app}} \cdot \nabla W_{i}^{\varepsilon} v+v \cdot Z_{i} \nabla u^{\mathrm{app}}+\varepsilon \partial_{z} p \nabla W_{i}^{\varepsilon}+\varepsilon \Delta W_{i}^{\varepsilon} v+2 \varepsilon \nabla W_{i}^{\varepsilon} \cdot \nabla v .
\end{gathered}
$$


Note that thanks to the crucial property (109), we have, using the notation $v_{h}=\left(v_{1}, v_{2}\right)$ for vectors of $v \in \mathbb{R}^{3}$, that

$$
\begin{aligned}
\mathcal{C}_{1}^{Z} & =\left(\partial_{i} u_{3}^{\mathrm{int}}-\varepsilon \partial_{t} W_{i}^{\varepsilon}\right) \partial_{z} v+\partial_{i}\left(u^{b}+u^{r}\right) \cdot \nabla v+\partial_{i} u_{h}^{\mathrm{int}} \cdot \nabla_{h} v-\varepsilon W_{i}^{\varepsilon} \partial_{z} u^{\mathrm{app}} \cdot \nabla v \\
(114) \quad & =\mathcal{O}(\varepsilon) \partial_{z} v+\partial_{i}\left(u^{b}+u^{r}\right) \cdot \nabla v+\partial_{i} u_{h}^{\mathrm{int}} \cdot \nabla_{h} v-\varepsilon W_{i}^{\varepsilon} \partial_{z} u^{\mathrm{app}} \cdot \nabla v
\end{aligned}
$$

By using that

$$
\varphi(z) \partial_{z} u^{b}=\mathcal{O}(1)
$$

since $\varphi(z)$ vanishes on the boundary and by using also the inequality (38), we get that

$$
\int_{0}^{T}\left\|\mathcal{C}^{Z}\right\|^{2} \lesssim \int_{0}^{T}\left(\varepsilon^{2}\|\nabla v\|^{2}+\varepsilon^{2}\|\nabla p\|^{2}+\left\|\nabla_{h} v\right\|^{2}\right) \lesssim D_{T, 0}(v, p)+\int_{0}^{T}\|v\|_{1}^{2}
$$

where in the second inequality we have used the property (111).

Finally, let us notice that

$$
\nabla \cdot\left(Z_{i} v\right)=\varepsilon \nabla W_{i}^{\varepsilon} \cdot \partial_{z} v:=d_{i} .
$$

A difficulty comes from the nonvanishing divergence of $Z_{i} v$. To estimate the solution of (113), we follow the same scheme as in the proof of Theorem 4. We use the same localization in frequencies. We begin with the small frequencies, which is actually the more difficult. We apply $\kappa^{s}\left(\varepsilon D_{y}\right)$ to (113) to get

$$
\mathcal{T}^{\varepsilon}\left(\kappa^{s} Z_{i} v, \kappa^{s} Z_{i} p\right)=\kappa^{s} Z_{i} F-\kappa^{s} \mathcal{C}^{Z}+\mathcal{C}^{s}
$$

where the commutator $\mathcal{C}^{s}$ satisfies the estimate

$$
\left\|\mathcal{C}^{s}\right\|^{2} \lesssim \varepsilon^{2}\|\nabla v\|_{1}^{2}+\|v\|_{1}^{2}
$$

We use the estimates (115), (118) and the standard energy estimate for (117) to obtain

$$
\begin{aligned}
& \left\|\kappa^{s} Z_{i}(T)\right\|^{2}+\varepsilon \int_{0}^{T}\left\|\kappa^{s} \nabla Z_{i} v\right\|^{2}+\left(\kappa^{s} \nabla Z_{i} p, \kappa^{s} Z_{i} v\right) \\
& \lesssim S^{\varepsilon}+D_{T, 0}(v, p)+\int_{0}^{T}\left(\|v\|_{1}^{2}+\varepsilon^{2}\|\nabla v\|_{1}^{2}+(F, v)_{1}\right)
\end{aligned}
$$

where the singular term $S^{\varepsilon}$ is defined as

$$
S^{\varepsilon}=\left|\left(Z_{i} v \cdot \partial_{z} u^{b}, Z_{i} v\right)\right|
$$

As in the proof of Proposition 7, we can estimate the singular term

$$
S^{\varepsilon} \lesssim \varepsilon r\left\|\nabla Z_{i} v\right\|^{2}
$$

and hence we can absorb it in the left-hand side. Next, we have to be careful with the term involving the pressure since $Z_{i} v$ is not divergence free. We write thanks to integration by parts and (116)

$$
\begin{aligned}
\left(\kappa^{s} \nabla Z_{i} p, \kappa^{s} Z_{i} v\right) & =\varepsilon\left(\kappa^{s} Z_{i} p, \kappa^{s} \nabla W_{i}^{\varepsilon} \cdot \partial_{z} v\right) \\
& =-\varepsilon\left(\kappa^{s} \partial_{z} Z_{i} p, \kappa^{s} \nabla W_{i}^{\varepsilon} \cdot v\right)-\varepsilon\left(\kappa^{s} \partial_{z} Z_{i} p, \kappa^{s} \nabla \partial_{z} W_{i}^{\varepsilon} \cdot v\right)
\end{aligned}
$$

and hence we get

$$
\left|\left(\kappa^{s} \nabla Z_{i} p, \kappa^{s} Z_{i} v\right)\right| \lesssim \varepsilon\|\nabla p\|_{1}\|v\|_{1}
$$

$4^{\text {e }}$ SÉRIE - TOME $41-2008-\mathrm{N}^{\mathrm{o}} 6$ 
so that we finally find

$$
\begin{aligned}
& \left\|\kappa^{s} Z_{i}(T)\right\|^{2}+\varepsilon \int_{0}^{T}\left\|\kappa^{s} \nabla Z_{i} v\right\|^{2} \\
& \lesssim D_{T, 0}(v, p)+\int_{0}^{T}\left(\|v\|_{1}^{2}+\varepsilon^{2}\|\nabla v\|_{1}^{2}+\varepsilon\|\nabla p\|_{1}\|v\|_{1}+(F, v)_{1}\right) .
\end{aligned}
$$

In a similar way, by combining the previous argument and the arguments in the proof of Proposition 5, we get in the high frequency region

$$
\begin{aligned}
& \left\|\kappa^{L} Z_{i}(T)\right\|^{2}+\int_{0}^{T}\left(\varepsilon^{-1}\left\|\kappa^{L} Z_{i} v\right\|^{2}+\varepsilon\left\|\kappa^{L} \nabla Z_{i} v\right\|^{2}\right) \\
& \lesssim D_{T, 0}(v, p)+\int_{0}^{T}\left(\|v\|_{1}^{2}+\varepsilon^{2}\|\nabla v\|_{1}^{2}++\varepsilon\|\nabla p\|_{1}\|v\|_{1}+(F, v)_{1}\right) .
\end{aligned}
$$

It remains the medium frequency estimates. In this range of frequency, we can lift the nonzero divergence and use the result of Proposition 8. Indeed, let us first establish some useful estimates on $d_{i}$. Thanks to (116), we have

$$
\int_{0}^{T}\left\|d_{i}\right\|^{2} \lesssim \varepsilon^{2} \int_{0}^{T}\|\nabla v\|^{2} \lesssim \varepsilon D_{T, 0}(v, p)
$$

and

$$
\int_{0}^{T}\left\|\nabla d_{i}\right\|^{2} \lesssim \varepsilon^{2} \int_{0}^{T}\left\|\nabla^{2} v\right\|^{2} \lesssim \varepsilon^{-2} D_{T, 0}(v, p)
$$

Moreover, we notice that

$$
\left\|d_{i}\right\|^{2} \lesssim \frac{1}{\Gamma^{2}}\left\|Z_{3} v\right\|^{2}+\varepsilon^{2}\left\|\partial_{z} v_{3}\right\|^{2} \lesssim \frac{1}{\Gamma^{2}}\left\|Z_{3} v\right\|^{2}+\varepsilon^{2}\left\|\nabla_{h} v_{h}\right\|^{2} \lesssim\left(\frac{1}{\Gamma^{2}}+\varepsilon^{2}\right) Y_{1}(v)
$$

and hence, by taking the time derivative, we also have

$$
\int_{0}^{T}\left\|\partial_{t} d_{i}\right\|^{2} \lesssim \int_{0}^{T}\left\|\partial_{t} v\right\|_{1}^{2}
$$

Now let us choose as before $D_{i}$ which satisfies the boundary condition (2) and such that

$$
\nabla \cdot D_{i}=d_{i} .
$$

By using (121), (122), (123), (124), we get

$$
\begin{aligned}
\int_{0}^{T}\left(\left\|D_{i}\right\|^{2}+\left\|\nabla D_{i}\right\|^{2}\right) & \lesssim \varepsilon D_{T, 0}(v, p), \\
\int_{0}^{T}\left\|\nabla^{2} D_{i}\right\|^{2} & \lesssim \varepsilon^{-2} D_{T, 0}(v, p), \\
\left\|\nabla D_{i}\right\|^{2} & \lesssim\left(\frac{1}{\Gamma^{2}}+\varepsilon^{2}\right)\|v\|_{1}^{2}, \\
\int_{0}^{T}\left(\left\|\partial_{t} D_{i}\right\|^{2}+\left\|\nabla \partial_{t} D_{i}\right\|^{2}\right) & \lesssim \int_{0}^{T}\left\|\partial_{t} v\right\|_{1}^{2} .
\end{aligned}
$$

To estimate $\kappa^{l} Z_{i} v$, we shall consider the equation satisfied by

$$
u=Z_{i} v-D_{i} .
$$


A very usefull remark already used to get (60) is that

$$
\varepsilon^{-2}\left\|\kappa^{l} D_{i}\right\|^{2} \lesssim\left\|\kappa^{l} \nabla D_{i}\right\|^{2} .
$$

Combined with (126)-(129), this gives very good estimates on $D_{i}$.

Thanks to (113), we get

$$
\mathcal{T}^{\varepsilon}\left(\kappa^{l} u, Z_{i} p\right)=\kappa^{l} Z_{i} F-\kappa^{l} \mathcal{C}^{Z}+\mathcal{C}^{l}+\mathcal{R}^{l}, \quad \nabla \cdot\left(\kappa^{l} u\right)=0
$$

where $\mathcal{C}^{l}$ is the commutator $\left[\kappa^{l}, \mathcal{T}^{\varepsilon}\right]$ and hence still satisfies the estimate (118) and $\mathcal{R}^{l}$ is defined by

$$
\mathcal{R}^{l}=\mathcal{T}^{\varepsilon}\left(\kappa^{l} D_{i}, 0\right) .
$$

Consequently, by combining (126), (127), (129) and (131), we get the estimate

$$
\int_{0}^{T}\left\|\mathcal{R}^{l}\right\|^{2} \lesssim \int_{0}^{T}\left(\varepsilon^{2}\left\|\partial_{t} v\right\|_{1}^{2}\right)+\varepsilon^{2} D_{T, 0}(v, p) .
$$

Next, since $u$ solves (132), we can use the result of Proposition 8 to get

$$
\begin{aligned}
& \left\|\kappa^{l} u(T)\right\|^{2}+\varepsilon^{2}\left\|\nabla\left(\kappa^{l} u\right)(T)\right\|^{2}+\int_{0}^{T}\left(\varepsilon^{-1}\left\|\kappa^{l} u\right\|^{2}+\varepsilon\left\|\kappa^{l} \nabla u\right\|^{2}\right) \\
& \lesssim e^{\gamma_{0} T}\left(\left\|\kappa^{l} u_{0}\right\|^{2}+\varepsilon^{2}\left\|\nabla\left(\kappa^{l} u_{0}\right)\right\|^{2}\right)+\varepsilon e^{\gamma_{0} T} \int_{0}^{T}\left(\|F\|_{1}^{2}+\left\|\kappa^{l} \mathcal{C}^{Z}\right\|^{2}+\left\|\mathcal{C}^{l}\right\|^{2}+\left\|\mathcal{R}^{l}\right\|^{2}\right) .
\end{aligned}
$$

Now, we can use (126), (128) and (131) and the fact that $u=Z_{i} v-D_{i}$ plus the estimates (115), (118) and (134). From now on, we restrict $\varepsilon$ and $T$ such that $\varepsilon e^{\gamma_{0} T} \leq 1$. This yields

$$
\lesssim e^{\gamma_{0} T}\left(\left\|v_{0}\right\|_{1}^{2}+\varepsilon^{2}\left\|\kappa^{l} \nabla v_{0}\right\|_{1}^{2}\right)+D_{T, 0}(v, p)+\int_{0}^{T}\left(\varepsilon e^{\gamma_{0} T}\|F\|_{1}^{2}+\|v\|_{1}^{2}+\varepsilon^{2}\|\nabla v\|_{1}^{2}+\varepsilon^{3}\left\|\partial_{t} v\right\|_{1}^{2}\right) .
$$

Note that by combining (119), (120), (135), we have actually proven that

$$
\begin{aligned}
& \|Z v\|^{2}+\int_{0}^{T}\left(\varepsilon\|\nabla Z v\|^{2}+\varepsilon^{-1}\left\|Z v^{H F}\right\|^{2}\right) \\
& \lesssim e^{\gamma_{0} T} Y_{1}\left(v_{0}\right)+D_{T, 0}(v, p)+\int_{0}^{T}\left(\|v\|_{1}^{2}+\varepsilon e^{\gamma_{0} T}\|F\|_{1}^{2}\right) .
\end{aligned}
$$

Next, since $u$ solves

$$
\mathcal{T}^{\varepsilon}\left(u, Z_{i} p\right)=Z_{i} F-\mathcal{C}^{Z}+\mathcal{R}, \quad \nabla \cdot u=0
$$

where

$$
\mathcal{R}=\mathcal{T}^{\varepsilon}\left(D_{i}, 0\right),
$$

we get thanks to the result of Lemma 11 that

$$
\begin{aligned}
& \varepsilon^{3}\|\nabla u(T)\|^{2}+\int_{0}^{T}\left(\varepsilon^{2}\left\|\partial_{t} u\right\|^{2}+\varepsilon^{4}\left\|\nabla^{2} Z v\right\|^{2}+\varepsilon^{2}\|\nabla Z p\|^{2}\right) \\
& \lesssim \varepsilon^{3}\left\|\nabla u_{0}\right\|^{2}+\int_{0}^{T}\left(\|u\|^{2}+\varepsilon^{2}\|\nabla u\|^{2}+\varepsilon^{2}\left\|\mathcal{C}^{Z}\right\|^{2}+\varepsilon^{2}\|\mathcal{R}\|^{2}\right)
\end{aligned}
$$


and hence by using (130) and (126), (127), (128), (129), we find

$$
\begin{aligned}
& \text { (138) } \quad \varepsilon^{3}\|\nabla Z v(T)\|^{2}+\int_{0}^{T}\left(\varepsilon^{2}\left\|\partial_{t} Z v\right\|^{2}+\varepsilon^{4}\left\|\nabla^{2} Z v\right\|^{2}+\varepsilon^{2}\|\nabla Z p\|^{2}\right) \\
& \lesssim \varepsilon^{3}\left(\left\|v_{0}\right\|_{1}^{2}+\left\|\nabla v_{0}\right\|_{1}^{2}\right)+D_{T, 0}(v, p)+\int_{0}^{T}\left(\|v\|_{1}^{2}+\varepsilon^{2}\|\nabla Z v\|^{2}+D_{T, 0}(v, p)+\varepsilon^{2}\|F\|^{2}\right) .
\end{aligned}
$$

To conclude, we can add (112) for $m=0$ and a large constant (independent of $\varepsilon$ ) times (136) plus (138) to get

$$
\begin{aligned}
\|v(T)\|_{1}^{2}+\varepsilon^{3}\|\nabla v(T)\|_{1}^{2}+ & D_{T, 1}(v, p) \\
& \lesssim Y_{1}\left(v_{0}\right)+\int_{0}^{T}\left((F, v)_{1}+\varepsilon\|\nabla p\|_{1}\|v\|_{1}+\|v\|_{1}^{2}+\varepsilon\|F\|_{1}^{2}\right) .
\end{aligned}
$$

Next, we use the Young inequality to write for every $\delta>0$

$$
\varepsilon\|\nabla p\|_{1}\|v\|_{1} \leq \frac{\delta}{2} \varepsilon^{2}\|\nabla p\|_{1}^{2}+C(\delta)\|v\|_{1}^{2}
$$

and we choose $\delta$ sufficiently small to absorb $\varepsilon^{2}\|\nabla p\|_{1}^{2}$ in $D_{T, 1}(v, p)$ so that we get

$$
\begin{aligned}
\|v(T)\|_{1}^{2}+\varepsilon^{3}\|\nabla v(T)\|_{1}^{2}+D_{T, 1}(v, p) & \\
& \lesssim e^{\gamma_{0} T} Y_{1}\left(v_{0}\right)+\int_{0}^{T}\left((F, v)_{1}+\|v\|_{1}^{2}+\varepsilon e^{\gamma_{0} T}\|F\|_{1}^{2}\right),
\end{aligned}
$$

and we conclude by using the Gronwall inequality as in the end of the proof of Theorem 4 .

It remains to estimate $\left\|\nabla_{h} Z_{i} v_{h}\right\|$ and $\left\|\nabla v_{3}\right\|$. We use again (137). The result of Theorem 12 gives

$$
\begin{aligned}
\varepsilon^{2}\left(\left\|\nabla_{h} u_{h}(T)\right\|^{2}+\|\right. & \left.\nabla w(T) \|^{2}\right) \\
& \lesssim\left\|u_{0}\right\|_{1, \varepsilon}^{2}+\int_{0}^{T}\left(\varepsilon\|F\|_{1}^{2}+\varepsilon\left\|\mathcal{C}^{Z}\right\|^{2}+\varepsilon\|\mathcal{R}\|^{2}+\|u\|^{2}+\varepsilon\|\nabla u\|^{2}\right)
\end{aligned}
$$

and hence we can use that $u=Z_{i} v-D_{I}$ and (126)-(129) and (115) to get

$\varepsilon^{2}\left(\left\|\nabla_{h} Z_{i} v(T)\right\|^{2}+\left\|\nabla Z_{i} v_{3}(T)\right\|^{2}\right) \lesssim Y_{1}\left(v_{0}\right)+D_{T, 1}(v, p)+\|v(T)\|_{1}^{2}+\int_{0}^{T}\left(\varepsilon\|F\|^{2}+\|v\|_{1}^{2}\right)$ and we can conclude since all the terms in the right-hand side have already been estimated.

We have given the proof for $m=1$, the general case follows by induction, this is left to the reader.

\section{Nonlinear stability}

In this section we prove the main theorem 2 . We introduce a new notation, namely for $f \in H_{\text {anis }}^{m}(\Omega)$, we denote for $z \in(0,1)$

$$
|f|_{m}^{2}(z)=\sum_{\alpha \in \mathbb{N}^{3},|\alpha| \leq m}\left|Z^{\alpha} f\right|_{L^{2}\left(\mathbb{T}_{a}^{2}\right)}
$$


where the integration only takes place in the $y$ variable. An important remark is that, for every $f \in H_{\text {anis }}^{m}(\Omega)$, we have by Sobolev imbedding

$$
|f(., z)|_{L^{\infty}\left(\mathbb{T}_{a}^{2}\right)} \leq|f(., z)|_{m} \quad m \geq 2 .
$$

Consequently by Leibniz' formula, we find

$$
|f g|_{m} \lesssim|f|_{m}|g|_{m}, \quad m \geq 2 .
$$

\subsection{Proof of Theorem 2}

For the proof of theorem 2, we recall that $u^{\text {app }}=u^{\text {int }}+u^{b}+u^{r}$, then we can see that $u^{\text {app }}$ is an approximate solution to (1) with an error term $F^{\varepsilon}$ which has size $\sqrt{\varepsilon}$ in $L^{\infty}\left(0, T ; L^{2}(\Omega)\right)$. Moreover, we can describe more precisely the structure of $F^{\varepsilon}$. Namely, we have

$$
F^{\varepsilon}=\partial_{t} u^{\mathrm{app}}+\frac{e \times u^{\mathrm{app}}}{\varepsilon}-\varepsilon \Delta u^{\mathrm{app}}+u^{\mathrm{app}} \cdot \nabla u^{\mathrm{app}}+\nabla p
$$

and we can see that $F^{\varepsilon}$ can be decomposed as

$$
F^{\varepsilon}=F^{\varepsilon, 1}+F^{\varepsilon, 2}
$$

with two types of terms. The first term $F^{\varepsilon, 1}$ contains boundary layer terms such as $u^{\text {app }} \cdot \nabla u^{b}$ and hence has an $L^{2}$ norm of size $\sqrt{\varepsilon}$ and is concentrated near $z=0$ or $z=1$. The other type is $F^{\varepsilon, 2}$ which has an $L^{2}$ norm of size $\varepsilon$ and which comes for instance from the time derivative of $\mathcal{Y}$.

For each $\varepsilon>0$, the existence theory for the Navier-Stokes system yields the existence and uniqueness of a solution $u^{\varepsilon}$ to (1) in $L^{\infty}\left(0, T^{\varepsilon} ; H^{1}(\Omega)\right)$ with the initial data $u^{\varepsilon, 0}$ on some time interval $T^{\varepsilon}>0$. Proving that the time $T^{\varepsilon}>0$ can be taken uniform in $\varepsilon$ and the convergence of $u^{\varepsilon}-\mathcal{L}\left(\frac{t}{\varepsilon}\right) w$ to zero will be done together. We set $v=u^{\varepsilon}-u^{\text {app }}$ where we recall that $u^{\text {app }}=u^{\text {int }}+u^{b}+u^{r}$. Note that $v$ depends on $\varepsilon$, but we drop this dependence in $\varepsilon$ in the notation. We find that $v$ solves

$$
\left\{\begin{array}{l}
\partial_{t} v+u^{\text {app }} \cdot \nabla v+v \cdot \nabla u^{\text {app }}+\frac{e \times v}{\varepsilon}-\varepsilon \Delta v+\nabla p=-F^{\varepsilon}-v \cdot \nabla v \\
\operatorname{div}(v)=0, \quad v=0 \text { on } \partial \Omega \quad v(t=0)=u^{\varepsilon, 0}-u^{\text {app }}(t=0) .
\end{array}\right.
$$

We start with the case $3 / 4<\alpha \leq 1$. Let us define

$$
T^{\varepsilon}=\sup \left\{t_{0} \mid \forall t \in\left[0, t_{0}\right], Y_{m}(v(t)) \leq C_{0}^{2} \varepsilon^{2 \alpha}\right\}
$$

for some big constant $C_{0}$. We recall that $Y_{m}(v(0)) \leq c^{2} \varepsilon^{2 \alpha}$ for some constant $c$, hence by continuity $T^{\varepsilon}>0$ with the choice $C_{0}>c$. Notice that (140) can be written as $\mathcal{T}^{\varepsilon}(v, p)=$ $-\left(F^{\varepsilon}+v \cdot \nabla v\right)$. Hence, we can use Theorem 13 to deduce that for $0<T<T^{\varepsilon}$, we have

$$
\begin{aligned}
Y_{T, m}(v)+D_{T, m}(v, p) \lesssim & e^{\gamma T}\left(Y_{m}\left(v_{0}\right)+\int_{0}^{T}\left(\kappa^{s}\left(F^{\varepsilon}+v \cdot \nabla v\right), \kappa^{s} v\right)_{m}+\right. \\
& \left.\varepsilon \int_{0}^{T}\left\|\left(F^{\varepsilon}+v \cdot \nabla v\right)\right\|_{m}^{2}\right) .
\end{aligned}
$$

We have to estimate the different terms appearing on the right-hand side of (141). We recall that $F^{\varepsilon}$ can be written as $F^{\varepsilon}=F^{\varepsilon, 1}+F^{\varepsilon, 2}$ (for simplicity of notation, we assume that $F^{\varepsilon, 1}$ 
is a boundary layer at $z=0$, the term at $z=1$ can be treated in a similar way by replacing $z$ by $1-z$ ). We have

$$
\begin{aligned}
\int_{0}^{T} & \left|\int_{\Omega}\left(\kappa^{s}\left(F^{\varepsilon, 1}+F^{\varepsilon, 2}\right), \kappa^{s} v\right)_{m}\right| \\
& \lesssim \int_{0}^{T}\left\|F^{\varepsilon, 2}\right\|_{m}^{2}+\|v\|_{m}^{2}+\int_{0}^{T} \int_{\mathbb{T}_{a}^{2}}\left(\int_{0}^{1}\left|z^{1 / 2} F^{\varepsilon, 1}\right|_{m} d z\left(\int_{0}^{1}\left|\partial_{z} v\right|_{m}^{2} d z\right)^{1 / 2}\right) d y d s \\
& \lesssim \int_{0}^{T}\left\|F^{\varepsilon, 2}\right\|_{m}^{2}+\|v\|_{m}^{2}+C \varepsilon^{3 / 2} \int_{0}^{T}\|\nabla v\|_{m} d s \\
& \lesssim \int_{0}^{T}\|v\|_{m}^{2}+C(1+T) \varepsilon^{2}+\frac{\varepsilon}{8} \int_{0}^{T}\|\nabla v\|_{m}^{2} d s
\end{aligned}
$$

Moreover, we also have

$$
\begin{aligned}
\int_{0}^{T}\left|\int_{\Omega}\left(\kappa^{s}(v \cdot \nabla v), \kappa^{s} v\right)_{m}\right| & \leq C \int_{0}^{T}\|v\|_{m}^{3 / 2}\|\nabla v\|_{m}^{3 / 2} d s \\
& \leq C T^{1 / 4} C_{0}^{3 / 2} \varepsilon^{3 / 2 \alpha-3 / 4}\left(\varepsilon \int_{0}^{T}\|\nabla v\|_{m}^{2}\right)^{3 / 4} \\
& \leq C T C_{0}^{6} \varepsilon^{6 \alpha-3}+\frac{1}{8} \varepsilon \int_{0}^{T}\|\nabla v\|_{m}^{2} d s
\end{aligned}
$$

The first term in the above estimate yields the restriction $6 \alpha-3>2 \alpha$ that is $\alpha>3 / 4$.

Besides,

$$
\begin{aligned}
\varepsilon \int_{0}^{T}\|v \cdot \nabla v\|_{m}^{2} & \lesssim \varepsilon \int_{0}^{T} \int_{0}^{1}\left|v_{h}\right|_{m}^{2}\left|\nabla_{h} v\right|_{m}^{2}+\left|v_{3}\right|_{m}^{2}\left|\partial_{z} v\right|_{m}^{2} d z d t \\
& \leq \varepsilon \int_{0}^{T}\left\|\partial_{z} v_{h}\right\|_{m}\left\|v_{h}\right\|_{m}\left\|\nabla_{h} v\right\|_{m}^{2}+\left\|\partial_{z} v_{3}\right\|_{m}\left\|v_{3}\right\|_{m}\left\|\partial_{z} v\right\|_{m}^{2} d t \\
& \leq \varepsilon \sup _{[0, T]}\left(\left\|v_{h}\right\|_{m}\left(\left\|\nabla_{h} v\right\|_{m}+\left\|\partial_{z} v_{3}\right\|_{m}\right) \int_{0}^{T}\|\nabla v\|_{m}^{2}\right. \\
& \leq \varepsilon^{-1} \sup _{[0, T]} Y_{m}(v(t))^{2} D_{T, m}(v, p) \leq C_{0}^{2} \varepsilon^{2 \alpha-1} D_{T, m}(v, p)
\end{aligned}
$$

and this term can be absorbed in the left-hand side if $\varepsilon$ is small enough since $\alpha>1 / 2$. Finally, we have

$$
\varepsilon \int_{0}^{T}\left\|F^{\varepsilon}\right\|_{m}^{2} \lesssim C T \varepsilon^{2}
$$

Hence, by Gronwall's lemma, we deduce that for all $T>0$, there exists an $\varepsilon_{0}$ such that for $\varepsilon<\varepsilon_{0}$, we can take $T^{\varepsilon}>T$ and we have

$$
\sup _{0 \leq t \leq T}\|v(t)\|^{2}+\int_{0}^{T} \varepsilon\|\nabla v\|^{2} \leq C_{T} \varepsilon^{2 \alpha}
$$

if $\alpha>3 / 4$.

REMARK 14. - The proof of Theorem 2 shows that for $\alpha=3 / 4$, we have a similar result but only on a finite time interval $\left(0, T_{0}\right)$ where $T_{0}$ depends on the initial data $w^{0}$ and the constant $c$ appearing in (19). In some sense, it appears from the proof of the theorem that the 
value $\alpha=3 / 4$ is critical. However, it may seem more natural to have a critical result at the value $\alpha=1 / 2$. But we were not able to prove Theorem 2 when $1 / 2<\alpha<3 / 4$. The main problem comes from estimating the nonlinear term $\int_{0}^{T}\left|\int_{\Omega}\left(\kappa^{s}(v . \nabla v), \kappa^{s} v\right)_{m}\right|$.

\section{Appendix A}

\section{Simple results about operator valued semi-classical pseudo-differential calculus}

We consider smooth symbols $A(y, \xi)$. Here, for each $y, \xi, A(y, \xi)$ is an operator from $H_{\xi}$ to $L^{2}\left(\mathbb{R}_{+}\right)$where $H_{\xi}$ is a closed subspace of $L^{2}\left(\mathbb{R}_{+}\right)$. We only need to consider operators associated with symbols of degree zero which basically verify

$$
\left|\partial_{y}^{\alpha} \partial_{\xi}^{\beta} A(y, \xi)\right|_{\mathcal{L}\left(H_{\xi}, L^{2}\left(\mathbb{R}_{+}\right)\right.} \leq C_{\alpha, \beta} .
$$

We associate to $A$ a semi-classical pseudo-differential operator acting on functions on $\mathbb{T}_{a}^{2} \times \mathbb{R}_{+}$defined by

$$
A\left(y, \varepsilon \partial_{y}\right) w=\mathrm{Op}_{A} w(y, z)=\sum_{k \in \mathbb{Z}^{2}} e^{i k \cdot y} A(y, \varepsilon k) \hat{w}(k, \cdot)(z)
$$

where $\hat{w}(k, z)$ are the Fourier coefficients of $w(\cdot, z)$, that is to say:

$$
w(y, z)=\sum_{k} e^{i k \cdot y} \hat{w}(k, z), \quad \hat{w}(k, z)=\int_{\mathbb{T}_{a}^{2}} e^{-i k \cdot y} w(y, z) d y ;
$$

here we assume that $d y$ is normalized such that $\int_{\mathbb{T}_{a}^{2}} d y=1$. We shall only give the proof of the properties that we have used; for more details, we refer for example to the book [8].

\section{A.1. Continuity in $L^{2}$}

Let us define

$$
|A|_{M, 0}=\sup _{y, \xi} \sup _{|\alpha| \leq M}\left|\partial_{y}^{\alpha} A(y, \xi)\right|_{\mathcal{L}\left(H_{\xi}, L^{2}\left(\mathbb{R}_{+}\right)\right)} .
$$

We also introduce the space $H \subset L^{2}\left(\mathbb{T}_{a}^{2} \times \mathbb{R}_{+}\right)$

$$
H=\left\{w \in L^{2}\left(\mathbb{T}_{a}^{2} \times \mathbb{R}_{+}\right), \quad \hat{w}(k, \cdot) \in H_{\varepsilon k}\right\} .
$$

We have the following result

Lemma 15. - There exists $C>0$ such that for every $\varepsilon, \varepsilon \in(0,1)$,

$$
\forall w \in H, \quad\left\|\mathrm{Op}_{A} w\right\|_{L^{2}\left(\mathbb{T}_{a}^{2} \times \mathbb{R}_{+}\right)} \leq C|A|_{M, 0}\|w\|_{L^{2}\left(\mathbb{T}_{a}^{2} \times \mathbb{R}_{+}\right)}
$$

for $M>2$.

$4^{\mathrm{e}}$ SÉRIE - TOME $41-2008-\mathrm{N}^{\mathrm{o}} 6$ 
Proof. - We follow the method to prove the boundedness of pseudo-differential operators in [31]. We expand $A(y, \varepsilon k) \hat{w}(k, \cdot)$ in Fourier series:

$$
A(y, \varepsilon k) \hat{w}(k, \cdot)=\sum_{l} e^{i l \cdot y} \hat{A}(l, \varepsilon k) \hat{w}(k, \cdot), \quad \hat{A}(l, \varepsilon k)=\int_{\mathbb{T}_{a}^{2}} e^{-i l \cdot y} A(y, \varepsilon k) d y .
$$

Since $A(y, \varepsilon k)$ is smooth in $y$, we have

$$
\left(1+|l|^{2}\right)^{N} \hat{A}(l, \varepsilon k)=\int_{\mathbb{T}_{a}^{2}}\left(I-\Delta_{y}\right)^{N}\left(e^{-i l \cdot y}\right) A(y, \varepsilon k) d y=\int_{\mathbb{T}_{a}^{2}} e^{-i l \cdot y}\left(I-\Delta_{y}\right)^{N} A(y, \varepsilon k) d y
$$

and hence we get

$$
|\hat{A}(l, \varepsilon k)|_{\mathcal{L}\left(L^{2}\left(\mathbb{R}_{+}\right)\right.} \lesssim \frac{1}{\left(1+|l|^{2}\right)^{N}}|A|_{2 N, 0}
$$

Since

$$
\mathrm{Op}_{A} w=\sum_{l} e^{i l \cdot y}\left(\sum_{k} e^{i k \cdot y} \hat{A}(l, \varepsilon k) \hat{w}_{k}\right),
$$

we get by using the Bessel identity that for $N>1$

$$
\left\|\mathrm{Op}_{A} w\right\| \lesssim \sum_{l}\left\|\sum_{k} e^{i k \cdot y} \hat{A}(l, \varepsilon k) \hat{w}_{k}\right\|=\sum_{l}\left(\sum_{k}\left|\hat{A}(l, \varepsilon k) w_{k}\right|^{2}\right)^{\frac{1}{2}}
$$

and hence thanks to (146), we obtain

$$
\left\|\mathrm{Op}_{A} w\right\| \lesssim|A|_{2 N, 0} \sum_{l} \frac{1}{\left(1+|l|^{2}\right)^{N}} \sum_{k}\left|w_{k}\right|^{2}
$$

which finally gives, by a new use of Bessel's identity

$$
\left\|\mathrm{Op}_{A} w\right\| \lesssim|A|_{N, 0}\|w\|
$$

This ends the proof.

\section{A.2. Product}

Here we only need to study the product of a differential operator and of a pseudodifferential operator of order 0 which is obvious and the product of a bounded Fourier multiplier and a pseudo-differential operator of order zero. Note that here we want to prove that the residual is small in $\varepsilon$.

Lemma 16. - Let $B(\xi) \in \mathcal{L}\left(L^{2}\right)$ and $A(y, \xi)$ be two symbols; then

$$
\mathrm{Op}_{B} \mathrm{Op}_{A} w=\mathrm{Op}_{B A} w+\varepsilon \mathrm{Op}_{R} w, \quad \forall w \in H
$$

where there exists $C$ such that for every $\varepsilon \in(0,1)$,

$$
\forall w \in H, \quad\left\|\mathrm{Op}_{R} w\right\|_{\left(L^{2}\left(\mathbb{T}_{a}^{2} \times \mathbb{R}_{+}\right)\right)} \leq C|B|_{0,1}|A|_{M, 0}\|w\|_{H}
$$

for $M>5$ where

$$
|B|_{0,1}=\sup _{\xi}\left|\nabla_{\xi} B(\xi)\right|_{\mathcal{L}\left(L^{2}\left(\mathbb{R}_{+}\right)\right.}
$$


Proof. - We write

$$
\begin{aligned}
\mathrm{Op}_{B} \mathrm{Op}_{A} w(y, \cdot) & =\sum_{k, l} \int_{\mathbb{T}_{a}^{2}} e^{i k\left(y-y^{\prime}\right)} e^{i l y^{\prime}} B(\varepsilon k) A\left(y^{\prime}, \varepsilon l\right) \hat{w}(l, \cdot) d y^{\prime} \\
& =\sum_{l} e^{i l y} \sum_{k} e^{i(k-l) y} B(\varepsilon k)\left(\int_{\mathbb{T}_{a}^{2}} e^{-i k y^{\prime}} A\left(y^{\prime}, \varepsilon l\right) d y^{\prime}\right) \hat{w}(l, \cdot) \\
& =\sum_{l} e^{i l y} \sum_{k} e^{i(k-l) y} B(\varepsilon k) \hat{A}(k, \varepsilon l) \hat{w}(l, \cdot) \\
& =\sum_{l} e^{i l y}\left(\sum_{k} e^{i k y} B(\varepsilon(k+l)) \hat{A}(k, \varepsilon l)\right) \hat{w}(l, \cdot) .
\end{aligned}
$$

Consequently, the symbol of $\mathrm{Op}_{B} \mathrm{Op}_{A}$ is $C$ defined by

$$
C(y, \varepsilon l)=\sum_{k} e^{i k y} B(\varepsilon(k+l)) \hat{A}(k, \varepsilon l) .
$$

Note that the object is well-defined since $B$ is uniformly bounded and $\hat{A}(k, \varepsilon l)$ is fastly decreasing in $k$. By Taylor's expansion, we can write

$$
B(\varepsilon(k+l))=B(\varepsilon l)+\varepsilon B^{1}(\varepsilon l, \varepsilon k) \cdot k, \quad B^{1}(\xi, \zeta)=\int_{0}^{1} D B(\xi+t \zeta) d t
$$

and hence we get

$$
\mathrm{Op}_{B} \mathrm{Op}_{A} w(y, \cdot)=\mathrm{Op}_{B A} w(y, \cdot)+\varepsilon \mathrm{Op}_{R} w(y, \cdot)
$$

where $R$ is defined by

$$
R(y, \xi)=\sum_{k} e^{i k \cdot y} B^{1}(\xi, \varepsilon k) \cdot k \hat{A}(k, \xi)
$$

Since $B^{1}$ is uniformly bounded and $\hat{A}$ is fastly decreasing, we easily get that $R$ satisfies (144). More precisely, we have

$$
|R|_{M, 0} \lesssim|B|_{0,1}|A|_{M+4,0}
$$

We end the proof by using Lemma 15 .

\section{A.3. Version with time dependence}

Here, we consider the case where for each $y, \xi, A(y, \xi)$ is an operator from $L^{2}\left(0, T ; H_{\xi}\right)$ or $L^{\infty}\left(0, T ; H_{\xi}\right)$ to $L^{2}\left((0, T) \times \mathbb{R}_{+}\right)$or $L^{\infty}\left((0, T) ; L^{2}\left(\mathbb{R}_{+}\right)\right)$. Let us set

$$
\|w\|_{T, 2}=\|w\|_{L^{2}\left([0, T], L^{2}\left(\mathbb{T}_{a}^{2} \times \mathbb{R}\right)_{+}\right)}, \quad\|w\|_{T, \infty}=\|w\|_{L^{\infty}\left([0, T], L^{2}\left(\mathbb{T}_{a}^{2} \times \mathbb{R}\right)_{+}\right)}
$$

and

$$
\begin{aligned}
\|A\|_{M, 0, T, 2} & =\sup _{y, \xi} \sup _{|\alpha| \leq M}\left|\partial_{y}^{\alpha} A(y, \xi)\right|_{\mathcal{L}\left(L^{2}\left([0, T], H_{\xi}\right), L^{2}\left([0, T], L^{2}\left(\mathbb{R}_{+}\right)\right)\right)} \\
\|A\|_{M, 0, T, \infty} & =\sup _{y, \xi} \sup _{|\alpha| \leq M}\left|\partial_{y}^{\alpha} A(y, \xi)\right|_{\mathcal{L}\left(L^{\infty}\left([0, T], H_{\xi}\right), L^{2}\left([0, T], L^{2}\left(\mathbb{R}_{+}\right)\right)\right)}, \\
\|B\|_{0,1, T, 2} & =\sup _{\xi}\left\|\nabla_{\xi} B\right\|_{\mathcal{L}\left(L^{2}\left([0, T], L^{2}\left(\mathbb{R}_{+}\right)\right)\right)}, \\
\|B\|_{0,1, T, \infty} & =\sup _{\xi}\left\|\nabla_{\xi} B\right\|_{\mathcal{L}\left(L^{\infty}\left([0, T], L^{\infty}\left(\mathbb{R}_{+}\right)\right)\right) .}
\end{aligned}
$$

Then we have the following properties: 
Lemma 17. - There exists $C>0$ such that

$$
\begin{aligned}
\forall w \in L^{2}([0, T], H), \quad\left\|\mathrm{Op}_{A} w\right\|_{T, 2} & \leq C\|A\|_{M, 0, T, 2}\|w\|_{T, 2}, \\
\left\|\mathrm{Op}_{A} w\right\|_{T, \infty} & \leq C\|A\|_{M, 0, T, \infty}\|w\|_{T, 2}
\end{aligned}
$$

and

$$
\mathrm{Op}_{B} \mathrm{Op}_{A} w=\mathrm{Op}_{B A} w+\varepsilon \mathrm{Op}_{R}
$$

with

$$
\begin{aligned}
\forall w \in L^{2}([0, T], H), \quad\left\|\mathrm{Op}_{R} w\right\|_{T, 2} & \leq C\|B\|_{0,1, T, 2}\|A\|_{M, 0, T, 2}\|w\|_{T, 2}, \\
\left\|\mathrm{Op}_{R} w\right\|_{T, \infty} & \leq C\|B\|_{0,1, T, \infty}\|A\|_{M, 0, T, \infty}\|w\|_{T, 2} .
\end{aligned}
$$

The proof can be obtained by the same method as in the previous version and we shall not detail it.

\section{Appendix B}

\section{The Leray projection}

\section{B.1. The case of a half space}

In this section, we study the symbol $\mathbb{P}_{+}(k)$ for $k \neq 0$. We look for a decomposition

$$
v=u+\left(\begin{array}{c}
i k p \\
\partial_{z} p
\end{array}\right), z>0, \quad i k \cdot u_{h}+\partial_{z} u_{3}=0, \quad u_{3}(0)=0
$$

and we set $\mathbb{P}_{+}(k) v=u$. It is convenient to introduce also $\mathbb{Q}_{+}(k)=I d-\mathbb{P}_{+}(k)$. We have the following properties:

Lemma 18. - $\quad$ i) The operator $\mathbb{P}_{+}(k)$ can be written for every $v \in L^{2}\left(\mathbb{R}_{+}\right)$as

$$
\mathbb{P}_{+}(k) v(z)=I_{h} v(z)-\int_{\mathbb{R}_{+}} K_{+}\left(k, z, z^{\prime}\right) v\left(z^{\prime}\right) d z^{\prime}, \quad \forall z \geq 0
$$

where

$$
I_{h} v(z)=\left(\begin{array}{l}
v_{h}(z) \\
0
\end{array}\right)
$$

and there exists $C>0$ such that the matrix $K_{+}\left(k, z, z^{\prime}\right)$ satisfies the estimate

$$
\forall k \neq 0, \quad\left|K_{+}\left(k, z, z^{\prime}\right)\right| \leq C|k|\left(e^{-|k|\left|z-z^{\prime}\right|}+e^{-|k|\left(z+z^{\prime}\right)}\right) .
$$

ii) There exists $C>0$ such that the operators $\mathbb{P}_{+}(k)$ satisfy for $k \neq 0$ the uniform estimates

$$
\forall v \in L^{2}\left(\mathbb{R}_{+}\right), \quad\left|\mathbb{P}_{+}(k) v\right|_{L^{2}\left(\mathbb{R}_{+}\right)} \leq C|v|_{L^{2}\left(\mathbb{R}_{+}\right)} .
$$

iii) Let $\kappa(\xi)$ be a smooth bounded function which vanishes in the vicinity of zero and $\chi(z)$ another smooth bounded function; then there exists $C>0$ such that we have the uniform estimate

$$
\forall \varepsilon \in(0,1), \forall v \in L^{2}\left(\mathbb{R}_{+}\right), \quad\left|\left[\chi(z), \mathbb{P}_{+}(k) \kappa(\varepsilon k)\right] v\right|_{L^{2}\left(\mathbb{R}_{+}\right)} \lesssim \varepsilon|v|_{L^{2}\left(\mathbb{R}_{+}\right)} .
$$

The precise expression of $K_{+}$will be given in the proof. 
Proof. - Note that ii) is a direct consequence of i) and the Schur Lemma since

$$
\sup _{z^{\prime} \geq 0} \int_{z \geq 0}\left|K_{+}\left(k, z, z^{\prime}\right)\right| \leq C, \quad \sup _{z \geq 0} \int_{z^{\prime} \geq 0}\left|K_{+}\left(k, z, z^{\prime}\right)\right| \leq C .
$$

Let us prove iii); we have

$$
\begin{aligned}
{\left[\chi(z), \mathbb{P}_{+}(k) \kappa(\varepsilon k)\right] v } & =-\left[\chi(z), \mathbb{Q}_{+}(k) \kappa(\varepsilon k)\right] v \\
& =\int_{z^{\prime}} K_{+}\left(k, z, z^{\prime}\right) \kappa(\varepsilon k)\left(\chi(z)-\chi\left(z^{\prime}\right)\right) v\left(z^{\prime}\right) d z^{\prime}
\end{aligned}
$$

and hence since $\chi$ is smooth, we get thanks to (148)

$$
\begin{aligned}
& \left|\int_{z^{\prime}} K_{+}\left(k, z, z^{\prime}\right) \kappa(\varepsilon k)\left(\chi(z)-\chi\left(z^{\prime}\right)\right) v\left(z^{\prime}\right) d z^{\prime}\right| \\
& \lesssim \int\left(|k| e^{-|k|\left|z-z^{\prime}\right|}\left|z-z^{\prime}\right|+|k| e^{-|k|\left(z+z^{\prime}\right)}\left(z+z^{\prime}\right)\right) \kappa(\varepsilon k)\left|v\left(z^{\prime}\right)\right| d z^{\prime} \\
& \lesssim \int\left(e^{-\frac{|k|}{2}\left|z-z^{\prime}\right|}+e^{-\frac{|k|}{2}\left(z+z^{\prime}\right)}\right) \kappa(\varepsilon k)\left|v\left(z^{\prime}\right)\right| d z^{\prime} \\
& \lesssim \int\left(e^{-\frac{c}{\varepsilon}\left|z-z^{\prime}\right|}+e^{-\frac{c}{\varepsilon}\left(z+z^{\prime}\right)}\right)\left|v\left(z^{\prime}\right)\right| d z^{\prime}
\end{aligned}
$$

for some $c>0$, where in the two last lines, we have used the inequality $X e^{-X} \lesssim e^{-\frac{X}{2}}$ for $X \geq 0$ and the fact that on the support of $\kappa$, we have $\varepsilon|k| \geq c>0$. The result follows by a new use of the Schur Lemma since

$$
\sup _{z^{\prime} \geq 0} \int_{z \geq 0} e^{-\frac{c}{\varepsilon}\left|z-z^{\prime}\right|}+e^{-\frac{c}{\varepsilon}\left(z+z^{\prime}\right)} d z \leq C \varepsilon, \quad \sup _{z \geq 0} \int_{z^{\prime} \geq 0} e^{-\frac{c}{\varepsilon}\left|z-z^{\prime}\right|}+e^{-\frac{c}{\varepsilon}\left(z+z^{\prime}\right)} d z^{\prime} \leq C \varepsilon .
$$

It remains to prove i). The result follows by an explicit computation. Thanks to (147), we find that $p$ solves the ODE

$$
\left(\partial_{z z}-|k|^{2}\right) p=i k v_{h}+\partial_{z} v_{3}, \quad \partial_{z} p(0)=v_{3}(0) .
$$

The unique bounded solution is given by

$$
\begin{aligned}
p(z)= & -\int_{z}^{+\infty}\left(i k \cdot v_{h}\left(z^{\prime}\right)+\partial_{z} v_{3}\left(z^{\prime}\right)\right) e^{-|k| z^{\prime}} \frac{\cosh (|k| z)}{|k|} d z^{\prime} \\
& -\int_{0}^{z}\left(i k \cdot v_{h}\left(z^{\prime}\right)+\partial_{z} v_{3}\left(z^{\prime}\right)\right) e^{-|k| z} \frac{\cosh \left(|k| z^{\prime}\right)}{|k|} d z^{\prime}-\frac{v_{3}(0)}{|k|} e^{-|k| z}
\end{aligned}
$$

and hence, after an integration by part, we find

$$
\begin{aligned}
p= & -\int_{z}^{+\infty} i k \cdot v_{h}\left(z^{\prime}\right) e^{-|k| z^{\prime}} \frac{\cosh (|k| z)}{|k|}+v_{3}\left(z^{\prime}\right) e^{-|k| z^{\prime}} \cosh (|k| z) d z^{\prime} \\
& -\int_{0}^{z} i k \cdot v_{h}\left(z^{\prime}\right) e^{-|k| z} \frac{\cosh \left(|k| z^{\prime}\right)}{|k|}-v_{3}\left(z^{\prime}\right) e^{-|k| z} \sinh \left(|k| z^{\prime}\right) d z^{\prime} .
\end{aligned}
$$

Note that this also yields

$$
\begin{aligned}
\partial_{z} p= & v_{3}(z)-\int_{z}^{+\infty} i k \cdot v_{h}\left(z^{\prime}\right) e^{-|k| z^{\prime}} \sinh (|k| z)+v_{3}\left(z^{\prime}\right)|k| e^{-|k| z^{\prime}} \sinh (|k| z) d z^{\prime} \\
& -\int_{0}^{z}-i k \cdot v_{h}\left(z^{\prime}\right) e^{-|k| z} \cosh \left(|k| z^{\prime}\right)+v_{3}\left(z^{\prime}\right)|k| e^{-|k| z} \sinh \left(|k| z^{\prime}\right) .
\end{aligned}
$$


Finally, since

$$
\mathbb{P}_{+} v=v-\left(\begin{array}{c}
i k p \\
\partial_{z} p
\end{array}\right)=I_{h} v-\int_{0}^{+\infty} K_{+}\left(k, z, z^{\prime}\right) v\left(z^{\prime}\right) d z^{\prime}
$$

it suffices to read the expression of $K_{+}$; we find

(151)

$$
K_{+}\left(k, z, z^{\prime}\right)=\left(\begin{array}{ccc}
\frac{k_{1}^{2}}{|k|} e^{-|k| z^{\prime}} \cosh (|k| z) & \frac{k_{1} k_{2}}{|k|} e^{-|k| z^{\prime}} \cosh (|k| z) & -i k_{1} e^{-|k| z^{\prime}} \cosh (|k| z) \\
\frac{k_{1} k_{2}}{|k|} e^{-|k| z^{\prime}} \cosh (|k| z) & \frac{k_{2}^{2}}{|k|} e^{-|k| z^{\prime}} \cosh (|k| z) & -i k_{2} e^{-|k| z^{\prime}} \cosh (|k| z) \\
-i k_{1} e^{-|k| z^{\prime}} \sinh (|k| z) & -i k_{2} e^{-|k| z^{\prime}} \sinh \left(|k| z^{\prime}\right) & -|k| e^{-|k| z^{\prime}} \sinh (|k| z)
\end{array}\right),
$$

for $0 \leq z<z^{\prime}$, and

$$
K_{+}\left(k, z, z^{\prime}\right)=\left(\begin{array}{ccc}
\frac{k_{1}^{2}}{|k|} e^{-|k| z} \cosh \left(|k| z^{\prime}\right) & \frac{k_{1} k_{2}}{|k|} e^{-|k| z} \cosh \left(|k| z^{\prime}\right) & i k_{1} e^{-|k| z} \sinh \left(|k| z^{\prime}\right) \\
\frac{k_{1} k_{2}}{|k|} e^{-|k| z} \cosh \left(|k| z^{\prime}\right) & \frac{k_{2}^{2}}{|k|} e^{-|k| z} \cosh \left(|k| z^{\prime}\right) & i k_{2} e^{-|k| z} \sinh \left(|k| z^{\prime}\right) \\
i k_{1} e^{-|k| z} \cosh \left(|k| z^{\prime}\right) & i k_{2} e^{-|k| z} \cosh \left(|k| z^{\prime}\right) & -|k| e^{-|k| z} \sinh \left(|k| z^{\prime}\right)
\end{array}\right),
$$

for $z>z^{\prime} \geq 0$. The estimate (148) follows immediately from the above expressions.

\section{B.2. The case of a strip.}

We now study the operator $\mathbb{P}(k)$, for $k \neq 0$. We now look for the decomposition

$$
v=u+\left(\begin{array}{c}
i k p \\
\partial_{z} p
\end{array}\right), z \in(0,1), \quad i k \cdot u_{h}+\partial_{z} u_{3}=0, \quad u_{3}(0)=0, u_{3}(1)=0 .
$$

Lemma 19. - $\quad$ i) The operator $\mathbb{P}(k)$ can be written for every $v \in L^{2}\left(\mathbb{R}_{+}\right)$as

$$
\mathbb{P}(k) v(z)=I_{h} v(z)-\int_{(0,1)} K\left(k, z, z^{\prime}\right) v\left(z^{\prime}\right) d z^{\prime}, \quad \forall z \geq 0
$$

where

$$
I_{h} v(z)=\left(\begin{array}{l}
v_{h}(z) \\
0
\end{array}\right)
$$

and there exists $C>0$ such that the matrix $K\left(k, z, z^{\prime}\right)$ satisfies the estimate

$$
\forall k \neq 0, \quad\left|K_{+}\left(k, z, z^{\prime}\right)\right| \leq C|k|\left(e^{-|k|\left|z-z^{\prime}\right|}+e^{-|k|\left(1-z+1-z^{\prime}\right)}\right) .
$$

ii) There exists $C>0$ such that the operators $\mathbb{P}(k)$ satisfy for $k \neq 0$ the uniform estimates

$$
\forall v \in L^{2}\left(\mathbb{R}_{+}\right), \quad|\mathbb{P}(k) v|_{L^{2}(0,1)} \leq C|v|_{L^{2}(0,1)} .
$$

iii) Let $\kappa(\xi)$ be a smooth bounded function which vanishes in the vicinity of zero and $\chi(z)$ another smooth bounded function; then there exists $C>0$ and we have the uniform estimate

$$
\forall \varepsilon \in(0,1), \forall v \in L^{2}(0,1), \quad\left|\left[\chi(z), \mathbb{P}_{+}(k) \kappa(\varepsilon k)\right] v\right|_{L^{2}(0,1)} \lesssim \varepsilon|v|_{L^{2}(0,1)} .
$$


Proof. - We now solve the ODE

$$
\partial_{z z} p-|k|^{2} p=i k \cdot v_{h}+\partial_{z} v_{3}, z \in(0,1), \quad \partial_{z} p(0)=v_{3}(0), \partial_{z} p(1)=v_{3}(1) .
$$

The explicit resolution gives

$$
\begin{aligned}
p= & \left.\int_{0}^{z}\left(i k \cdot v_{h}+\partial_{z} v_{3}\right)\right) \frac{\sinh \left(|k|\left(z-z^{\prime}\right)\right)}{|k|} d z^{\prime} \\
& -\int_{0}^{1}\left(i k \cdot v_{h}+\partial_{z} v_{3}\right) \frac{\cosh (\mid k]\left(1-z^{\prime}\right)}{|k| \sinh |k|} \cosh (|k| z) d z^{\prime} \\
& -v_{3}(0) \frac{\cosh (|k|(z-1)}{|k| \sinh |k|}+v_{3}(1) \frac{\cosh (|k| z)}{|k| \sinh |k|}
\end{aligned}
$$

and hence after an integration by parts, we find

$$
\begin{aligned}
p= & -\int_{z}^{1}\left(i k \cdot v_{h} \frac{\cosh \left(|k|\left(1-z^{\prime}\right)\right) \cosh (|k| z)}{|k| \sinh |k|}+v_{3} \frac{\sinh \left(|k|\left(1-z^{\prime}\right)\right)}{\sinh |k|} \cosh (|k| z)\right) d z^{\prime} \\
& +\int_{0}^{z}\left(i k \cdot v_{h}\left(\frac{\sinh \left(|k|\left(z-z^{\prime}\right)\right)}{|k|}-\frac{\cosh \left(|k|\left(1-z^{\prime}\right)\right)}{|k| \sinh |k|} \cosh (|k| z)\right)\right. \\
& \left.+v_{3}\left(\cosh \left(|k|\left(z-z^{\prime}\right)\right)-\frac{\sinh \left(|k|\left(1-z^{\prime}\right)\right)}{\sinh |k|} \cosh (|k| z)\right)\right) d z^{\prime}
\end{aligned}
$$

Note that we have used the fact that

$$
v_{3}(0)\left(\frac{\cosh |k|}{|k| \sinh |k|} \cosh (|k| z)-\frac{\sinh (|k| z)}{|k|}-\frac{\cosh (|k|(z-1)}{|k| \sinh |k|}\right)=0 .
$$

Finally, we can rewrite the pressure under the more convenient form

$$
\begin{aligned}
p= & -\int_{z}^{1} i \frac{k}{|k|} \cdot v_{h}\left(\frac{e^{-|k| z^{\prime}} \cosh (|k| z)}{1-e^{-2|k|}}+\frac{e^{-|k|\left(2-z^{\prime}\right)} \cosh (|k| z)}{1-e^{-2|k|}}\right) d z^{\prime} \\
& -\int_{z}^{1} v_{3}\left(\frac{e^{-|k| z^{\prime}} \cosh (|k| z)}{1-e^{-2|k|}}-\frac{e^{-|k|\left(2-z^{\prime}\right)} \cosh (|k| z)}{1-e^{-2|k|}}\right) d z^{\prime} \\
& -\int_{0}^{z} i \frac{k}{|k|} \cdot v_{h}\left(\frac{e^{-|k| z} \cosh \left(|k| z^{\prime}\right)}{1-e^{-2|k|}}+\frac{e^{-|k|(2-z)} \cosh \left(|k| z^{\prime}\right)}{1-e^{-2|k|}}\right) d z^{\prime} \\
& +\int_{0}^{z} v_{3}\left(\frac{e^{-|k| z} \sinh \left(|k| z^{\prime}\right)}{\left(1-e^{-2|k|}\right)}+\frac{e^{-|k|(2-z)} \sinh \left(|k| z^{\prime}\right)}{1-e^{-2|k|}}\right) d z^{\prime} .
\end{aligned}
$$

By taking the derivative with respect to $z$, we also find

$$
\begin{aligned}
& \partial_{z} p=v_{3}(z)-\int_{z}^{1} i \frac{k}{|k|} \cdot v_{h}|k|\left(\frac{e^{-|k| z^{\prime}} \sinh (|k| z)}{1-e^{-2|k|}}+\frac{e^{-|k|\left(2-z^{\prime}\right)} \sinh (|k| z)}{1-e^{-2|k|}}\right) d z^{\prime} \\
& -\int_{z}^{1} v_{3}|k|\left(\frac{e^{-|k| z^{\prime}} \sinh (|k| z)}{1-e^{-2|k|}}-\frac{e^{-|k|\left(2-z^{\prime}\right)} \sinh (|k| z)}{1-e^{-2|k|}}\right) d z^{\prime} \\
& -\int_{0}^{z} i \frac{k}{|k|} \cdot v_{h}|k|\left(-\frac{e^{-|k| z} \cosh \left(|k| z^{\prime}\right)}{1-e^{-2|k|}}+\frac{e^{-|k|(2-z)} \cosh \left(|k| z^{\prime}\right)}{1-e^{-2|k|}}\right) d z^{\prime} \\
& +\int_{0}^{z} v_{3}|k|\left(-\frac{e^{-|k| z} \sinh \left(|k| z^{\prime}\right)}{\left(1-e^{-2|k|}\right)}+\frac{e^{-|k|(2-z)} \sinh \left(|k| z^{\prime}\right)}{1-e^{-2|k|}}\right) d z^{\prime} .
\end{aligned}
$$


Consequently, since

$$
\mathbb{P} v=v-\left(\begin{array}{c}
i k p \\
\partial_{z} p
\end{array}\right)=I_{h} v-\int_{0}^{+\infty} K\left(k, z, z^{\prime}\right) v\left(z^{\prime}\right) d z^{\prime},
$$

we find

$$
\begin{aligned}
& K\left(k, z, z^{\prime}\right)= \\
& \text { (156) } \frac{1}{1-e^{-2|k|}}\left(\begin{array}{cc}
\frac{k_{1}^{2}}{|k|} a_{+}\left(k, z, z^{\prime}\right) & \frac{k_{1} k_{2}}{|k|} a_{+}\left(k, z, z^{\prime}\right)-i k_{1} a_{-}\left(k, z, z^{\prime}\right) \\
\frac{k_{1} k_{2}}{|k|} a_{+}\left(k, z, z^{\prime}\right) & \frac{k_{2}^{2}}{|k|} a_{+}\left(k, z, z^{\prime}\right)-i k_{2} a_{-}\left(k, z, z^{\prime}\right) \\
-i k_{1} b_{+}\left(k, z, z^{\prime}\right) & -i k_{2} b_{+}\left(k, z, z^{\prime}\right)-|k| b_{-}\left(k, z, z^{\prime}\right)
\end{array}\right), 0 \leq z<z^{\prime}, \\
& (157)=\frac{1}{1-e^{-2|k|}}\left(\begin{array}{ccc}
\frac{k_{1}^{2}}{|k|} a_{+}\left(k, z^{\prime}, z^{\prime}\right) & \frac{k_{1} k_{2}}{|k|} a_{+}\left(k, z^{\prime}, z\right) & -i k_{1} b_{+}\left(k, z^{\prime}, z\right) \\
\frac{k_{1} k_{2}}{|k|} a_{+}\left(k, z^{\prime}, z\right) & \frac{k_{2}^{2}}{|k|} a_{+}\left(k, z^{\prime}, z\right) & -i k_{2} b_{+}\left(k, z^{\prime}, z\right) \\
i k_{1} a_{-}\left(k, z^{\prime}, z\right) & i k_{2} a_{-}\left(k, z^{\prime}, z\right) & -|k| b_{-}\left(k, z^{\prime}, z\right)
\end{array}\right), 0 \leq z^{\prime}<z
\end{aligned}
$$

where

$$
\begin{aligned}
& a_{+}\left(k, z, z^{\prime}\right)=e^{-|k| z^{\prime}} \cosh (|k| z)+e^{-|k|\left(2-z^{\prime}\right)} \cosh (|k| z), \\
& a_{-}\left(k, z, z^{\prime}\right)=e^{-|k| z^{\prime}} \cosh (|k| z)-e^{-|k|\left(2-z^{\prime}\right)} \cosh (|k| z), \\
& b_{+}\left(k, z, z^{\prime}\right)=e^{-|k| z^{\prime}} \sinh (|k| z)+e^{-|k|\left(2-z^{\prime}\right)} \sinh (|k| z), \\
& b_{-}\left(k, z, z^{\prime}\right)=e^{-|k| z^{\prime}} \sinh (|k| z)-e^{-|k|\left(2-z^{\prime}\right)} \sinh (|k| z) .
\end{aligned}
$$

Thanks to (156), (157), the estimate (153) follows easily and next, we obtain ii) and iii) as previously.

\section{B.3. Estimate of $\mathbb{P}-\mathbb{P}_{+}$}

Lemma 20. - Let $\kappa(\xi)$ be a smooth bounded function which vanishes in the vicinity of zero and $\chi_{1}(z), \chi_{2}(z)$ two smooth bounded functions which are compactly supported in $[0,1)$; then there exist $C>0$ and $c>0$ such that we have the uniform estimate

$$
\forall \varepsilon \in(0,1), \forall v \in L^{2}\left(\mathbb{R}_{+}\right), \quad\left|\chi_{1}\left(\mathbb{P}-\mathbb{P}_{+}\right) \kappa(\varepsilon k) \chi_{2} v\right|_{L^{2}\left(\mathbb{R}_{+}\right)} \leq C e^{-\frac{\varepsilon}{\varepsilon}}|v|_{L^{2}\left(\mathbb{R}_{+}\right)}
$$

Proof. - We can use the explicit expressions given by (156), (157) and (151), (152). This yields

$$
\begin{aligned}
& \left|\chi_{1}(z)\left(\mathbb{P}-\mathbb{P}_{+}\right) \kappa(\varepsilon k) \chi_{2}(z) v\right| \\
\leq & \int_{0}^{+\infty} \chi_{1}(z) \chi_{2}\left(z^{\prime}\right) \kappa(\varepsilon k)\left|v\left(z^{\prime}\right)\right|\left(|k| e^{-\left|k \| z-z^{\prime}\right|}\left(\frac{1}{1-e^{-2|k|}}-1\right)+|k| e^{-2|k|+|k| z+|k| z^{\prime}}\right) d z^{\prime}
\end{aligned}
$$


and hence if the support of $\kappa(\xi)$ is in $|\xi| \geq r>0$ and the supports of $\chi$ and $\chi^{\prime}$ are in $[0, \delta]$, $\delta<1$, we find

$$
\begin{aligned}
\mid \chi_{1}(z)\left(\mathbb{P}-\mathbb{P}_{+}\right) & \kappa(\varepsilon k) \chi_{2}(z) v \mid \\
& \leq \int_{0}^{+\infty} \chi_{1}(z) \chi_{2}\left(z^{\prime}\right) \kappa(\varepsilon k)\left|v\left(z^{\prime}\right)\right|\left(|k| e^{-|k|\left|z-z^{\prime}\right|} e^{\frac{-2 r}{\varepsilon}}+|k| e^{-2|k|(1-\delta)}\right) d z^{\prime} \\
& \lesssim \int_{0}^{+\infty} \chi_{1}(z) \chi_{2}\left(z^{\prime}\right) \kappa(\varepsilon k)\left|v\left(z^{\prime}\right)\right| e^{-\frac{r}{\varepsilon}} d z^{\prime}
\end{aligned}
$$

The estimate (158) follows by using again the Schur Lemma.

\section{REFERENCES}

[1] A. Babin, A. Mahalov, B. Nicolaenko, Global splitting, integrability and regularity of 3D Euler and Navier-Stokes equations for uniformly rotating fluids, European J. Mech. B Fluids 15 (1996), 291-300.

[2] A. Babin, A. Mahalov, B. Nicolaenko, Regularity and integrability of 3D Euler and Navier-Stokes equations for rotating fluids, Asymptot. Anal. 15 (1997), 103-150.

[3] A. J. Bourgeois, J. T. Beale, Validity of the quasigeostrophic model for large-scale flow in the atmosphere and ocean, SIAM J. Math. Anal. 25 (1994), 1023-1068.

[4] J.-Y. Chemin, B. Desjardins, I. Gallagher, E. Grenier, Mathematical geophysics, Oxford Lecture Series in Math. and its Appl. 32, The Clarendon Press Oxford University Press, 2006.

[5] T. Colin, P. Fabrie, Équations de Navier-Stokes 3-D avec force de Coriolis et viscosité verticale évanescente, C. R. Acad. Sci. Paris Sér. I Math. 324 (1997), 275-280.

[6] B. Desjardins, E. Dormy, E. Grenier, Stability of mixed Ekman-Hartmann boundary layers, Nonlinearity 12 (1999), 181-199.

[7] B. Desjardins, E. Grenier, Linear instability implies nonlinear instability for various types of viscous boundary layers, Ann. Inst. H. Poincaré Anal. Non Linéaire 20 (2003), 87-106.

[8] M. Dimassi, J. Suöstrand, Spectral asymptotics in the semi-classical limit, London Math. Soc. Lecture Note Series 268, Cambridge Univ. Press, 1999. 
[9] P. F. Embid, A. J. Majda, Averaging over fast gravity waves for geophysical flows with arbitrary potential vorticity, Comm. Partial Differential Equations 21 (1996), 619658.

[10] A. Friedman, Partial differential equations of parabolic type, Prentice-Hall Inc., 1964.

[11] G. P. GALDI, An introduction to the mathematical theory of the Navier-Stokes equations. Vol. I, Springer Tracts in Natural Philosophy 38, Springer, 1994.

[12] I. Gallagher, Applications of Schochet's methods to parabolic equations, J. Math. Pures Appl. 77 (1998), 989-1054.

[13] F. Gallaire, F. Rousset, Spectral stability implies nonlinear stability for incompressible boundary layers, Indiana Univ. Math. J. 57 (2008), 1959-1975.

[14] H. Greenspan, The theory of rotating fluids, Cambridge Monographs on Mechanics and Applied Mathematics, 1969.

[15] E. Grenier, Oscillatory perturbations of the Navier-Stokes equations, J. Math. Pures Appl. 76 (1997), 477-498.

[16] E. Grenier, On the nonlinear instability of Euler and Prandtl equations, Comm. Pure Appl. Math. 53 (2000), 1067-1091.

[17] E. Grenier, N. Masmoudi, Ekman layers of rotating fluids, the case of well prepared initial data, Comm. Partial Differential Equations 22 (1997), 953-975.

[18] E. Grenier, F. Rousset, Stability of one-dimensional boundary layers by using Green's functions, Comm. Pure Appl. Math. 54 (2001), 1343-1385.

[19] O. GuÈs, Perturbations visqueuses de problèmes mixtes hyperboliques et couches limites, Ann. Inst. Fourier (Grenoble) 45 (1995), 973-1006.

[20] D. Henry, Geometric theory of semilinear parabolic equations, Lecture Notes in Math. 840, Springer, 1981.

[21] D. LiLly, On the instability of Ekman boundary flow, J. Atmos. Sci. (1966), 481-494.

[22] N. Masmoudi, The Euler limit of the Navier-Stokes equations, and rotating fluids with boundary, Arch. Rational Mech. Anal. 142 (1998), 375-394.

[23] N. Masmoudi, Ekman layers of rotating fluids: the case of general initial data, Comm. Pure Appl. Math. 53 (2000), 432-483.

[24] G. MÉtivier, K. Zumbrun, Large viscous boundary layers for noncharacteristic nonlinear hyperbolic problems, Mem. Amer. Math. Soc. 175, 2005.

[25] J. Pedlosky, Geophysical fluid dynamics, Springer, 1979.

[26] F. Rousset, Stability of large Ekman boundary layers in rotating fluids, Arch. Ration. Mech. Anal. 172 (2004), 213-245.

[27] F. Rousset, Characteristic boundary layers in real vanishing viscosity limits, J. Differential Equations 210 (2005), 25-64.

[28] F. Rousset, Stability of large amplitude Ekman-Hartmann boundary layers in MHD: the case of ill-prepared data, Comm. Math. Phys. 259 (2005), 223-256.

[29] M. Sammartino, R. E. Caflisch, Zero viscosity limit for analytic solutions of the Navier-Stokes equation on a half-space. II. Construction of the Navier-Stokes solution, Comm. Math. Phys. 192 (1998), 463-491.

[30] S. Schochet, Fast singular limits of hyperbolic PDEs, J. Differential Equations 114 (1994), 476-512. 
[31] M. E. Taylor, Pseudodifferential operators, Princeton Mathematical Series 34, Princeton University Press, 1981.

(Manuscrit reçu le 15 octobre 2007; accepté, après révision, le 5 mai 2008.)

\author{
Nader Masmoudi \\ Courant Institute of Mathematical Sciences \\ 251 Mercer Street \\ New York, NY 10012-1185, U.S.A. \\ E-mail: masmoudi@courant.nyu.edu \\ Frédéric RousseT \\ IRMAR, \\ Universite de Rennes 1, \\ Campus de Beaulieu, \\ 35042 Rennes Cedex, France \\ E-mail: frederic.rousset@univ-rennes1.fr
}

